\title{
Pearls, Perils, and Pitfalls in the Use of the Electroencephalogram
}

\author{
Omkar N. Markand, M.D., F.R.C.P.C.1
}

Objectives: Upon completion of this article, the reader will understand "normal abnormalities" in the normal EEG, the EEG in encephalopathies, the proper identification of diagnostic epileptiform abnormalities, the classic patterns of infantile childhood and adult epilepsies, the EEG patterns morphologically similar to interictal/ictal epileptiform discharges but unrelated to epilepsy, and neonatal EEG abnormalities.

Accreditation: The Indiana University School of Medicine is accredited by the Accreditation Council for Continuing Medical Education to provide continuing medical education for physicians.

Credit: The Indiana University School of Medicine designates this educational activity for a maximum of 1.0 hours in category one credit toward the AMA Physicians Recognition Award. Each physician should claim only those hours of credit that he/she actually spent in the educational activity.

Disclosure: Statements have been obtained regarding the author's relationships with financial supporters of this activity. There is no apparent conflict of interest related to the context of participation of the author of this article.

Neurological Office Procedures; Editor in Chief, Karen L. Roos, M.D.; Guest Editor, Karen L. Roos, M.D. Seminars in Neurology, Volume 23, Number 1, 2003. Address for correspondence and reprint requests: Omkar N. Markand, M.D., Indiana University School of Medicine, 550 North University Blvd., Room 1711, Indianapolis, IN 46202. ${ }^{1}$ Department of Neurology, Indiana University School of Medicine, Indianapolis, Indiana. Copyright (C) 2003 by Thieme Medical Publishers, Inc., 333 Seventh Avenue, New York, NY 10001, USA. Tel: +1(212) 584-4662. 02718235,p;2003,23,01,007,046,ftx,en;sin00226x. 
Electroencephalography (EEG) is the technique of recording from the scalp the spontaneous electrical activity of the brain and correlating it to the underlying brain function. Since the first recording of a human EEG in 1929 by Hans Berger, improvement in electronics and technology has made EEG one of the most widely used laboratory tests for clinical evaluation of neurologic disorders. However, in the past three decades with continuing advances in neuroimaging, particularly magnetic resonance imaging (MRI), the role of clinical EEG has become restricted and progressively more focused. Its major utility at present is in the evaluation of focal and diffuse encephalopathies, comatose conditions, epileptic disorders, and cerebral disorders affecting neonates and infants. The present article is not an attempt to describe EEG comprehensively in normal subjects and in different disease processes but to highlight its usefulness/limitation and emphasize precautions/care needed in its optimal utility. The subject will be discussed under seven sections: EEG in normal subjects, EEG in patients with altered mental status or diffuse encephalopathies, EEG in focal or lateralized cerebral hemispheric lesions, EEG in paroxysmal disorders, EEG in generalized epilepsies, EEG in neonates, and EEG in status epilepticus.

\section{EEG IN NORMAL SUBJECTS}

The EEG in the normal awake child and adult is well known and needs no detailed description. The following are points of emphasis:

1. Alpha rhythm in the two hemispheres is very similar in frequency. A consistent difference of even 0.5 to $1.0 \mathrm{cps}$ on the two sides is significant; the side showing a slower frequency may have a hemispheric dysfunction. Amplitude asymmetry is of relatively less significance, unless the asymmetry is prominent. In general, the alpha rhythm is higher in amplitude over the right hemisphere. If the amplitude of the alpha rhythm on the right side is more than $1 \frac{1}{2}$ times that on the left side, the asymmetry is usually regarded as significant. When the alpha rhythm is over $25 \%$ higher in amplitude on the left side than the right side, this constitutes a significant asymmetry. ${ }^{1}$

2. Significant theta activity ( 4 to $7 \mathrm{~Hz}$ ) is present in the EEG of children and adolescents. Delta activity in the awake tracing is rarely seen after the age of 5 years. A common EEG pattern in adolescents is the presence of intermittent delta waves intermixed with alpha rhythm over the posterior head regions, the socalled "slow waves of youth."

3. The EEG during non-rapid eye movement (NREM) sleep in children shows very prominent spikelike vertex sharp transients, which are often mistaken for epileptiform activity by EEG inter- preters inexperienced with children's EEGs (Fig. 1). Similarly, positive occipital sharp transients (POSTs), when high in amplitude and sharp in configuration, can be easily misinterpreted as abnormal spikes, especially in linkages where occipital electrodes are connected to input terminal 2 (grid 2) of the amplifier (e.g., "double banana run").

4. In a small proportion of normal adult subjects, clearly identifiable and countable alpha rhythm may be entirely absent. The background may consist of irregular mixtures of low amplitude $(<20 \mu \mathrm{V})$ activities, mostly from 5.0 to 30.0 cps without a dominant frequency. Such low-voltage EEGs have been studied in detail. ${ }^{2}$ The EEG is reactive to various physiologic stimuli such as sleep, drugs, and pathologic processes. In over half of the patients with low-voltage EEGs, hyperventilation may bring out an alpha rhythm. During sleep, normal activities such as vertex sharp transients and sleep spindles may be generated. It is essential that low-voltage tracings be clearly distinguished from EEGs showing electrocerebral inactivity, which have a grave prognosis. These EEGs lack reactivity and lability, and with increased instrumental sensitivities show no electrical activity of cerebral origin. Low-voltage EEGs are generally considered to be a normal variant occurring in 7 to $10 \%$ of normal subjects over the age of 20 years. The low-voltage EEG does not correlate with neurologic or psychiatric disease.

5. Changes in the EEG during normal senescence has been described in detail. ${ }^{3-5}$ The most frequent change is the slowing of the alpha frequency. By the age of 70 years, the mean alpha frequency decreases to 9.0 to $9.5 \mathrm{cps}$ and decreases further to 8.5 to $9.0 \mathrm{cps}$ beyond the age of 80 years. In healthy elderly subjects, even at or over the age of 100 years, the frequency of the alpha rhythm remains well above 8.0 cps. ${ }^{6,7}$ Therefore, an average alpha frequency of less than $8.0 \mathrm{cps}$ measured with the patient fully alert must be considered abnormal in elderly patients at all ages.

Another EEG finding is the presence of isolated transients of irregular focal slowing in the theta-delta frequency range over the anterior temporal region, reported in $40 \%$ of healthy elderly subjects. $4,5,8$ They are most frequent over the left temporal area particularly during drowsiness (Fig. 2). Sometimes poorly defined sharp waves are interspersed with focal slow components. The left-sided accentuation of this activity remains unexplained. Such intermittent slow activity, with or without sharp components over the temporal region, has no correlation with intellectual or cognitive functioning or presence of a seizure disorder. More recent investigations suggest that the temporal slowing in the awake tracing may, in fact, not be the inevitable con- 
A1-T3

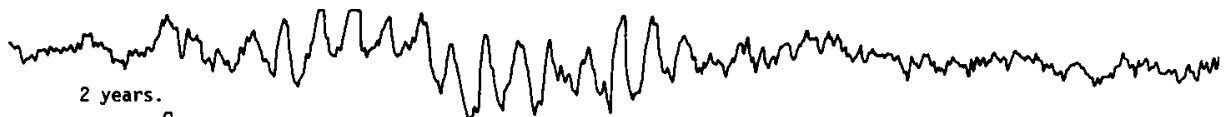

T3-C3

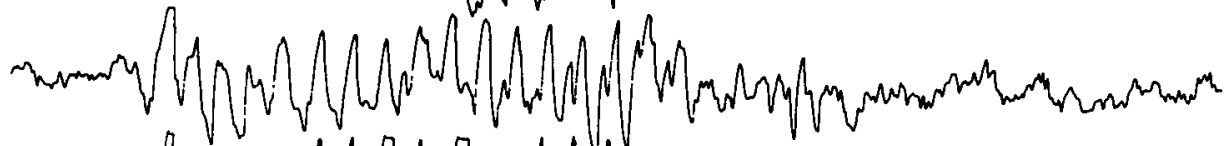

C3-C

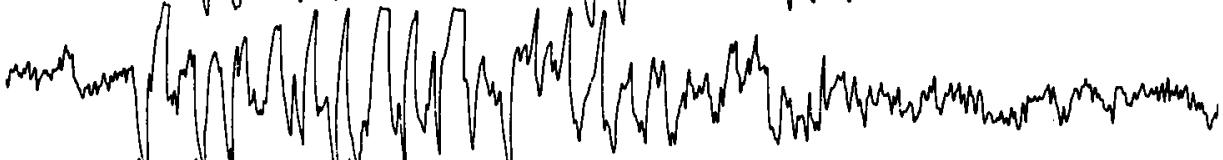

C4-T4

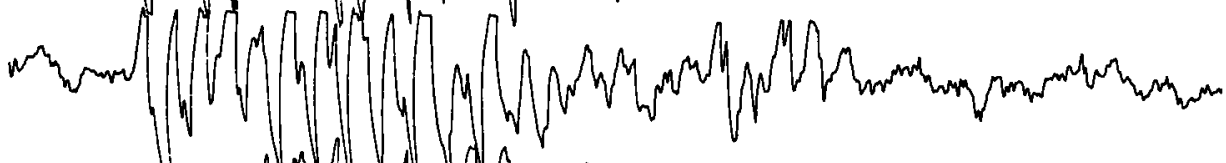

T4-A2

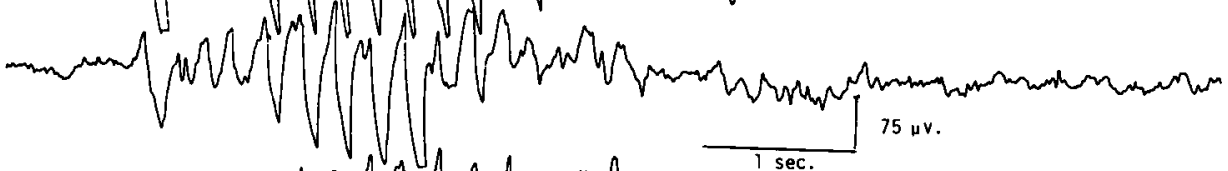

A2-A1

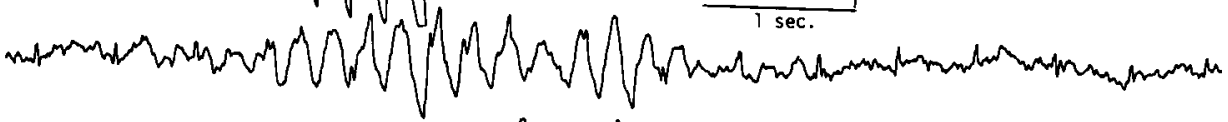

O1-A1

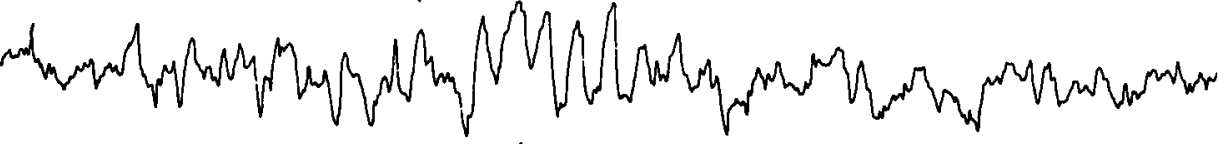

O2-A2

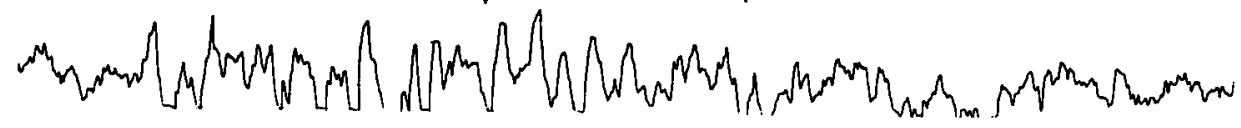

Figure 1 EEG of a 2-year-old child with very prominent spikelike vertex sharp transients.

sequence of advancing age. In neurologically and psychologically normal septuagenarians, Katz and Horowitz ${ }^{9}$ found that the focal slow activity was seen in only $17 \%$ of records and when present occupied less than $1 \%$ of the tracing. Hence, intermittent temporal theta-delta activity occupying only a small proportion of the wake tracing should be considered as a normal aging phenomena. When the temporal slow activity comprises more delta than theta slow waves, which either recur frequently or occur in long runs and are widespread in distribution, a dementing process or focal lesion has to be seriously considered. Diffuse theta-delta

$61 \mathrm{yr}$.

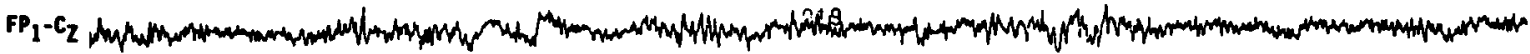
$F_{2}-C_{2} M_{2} H_{1}$

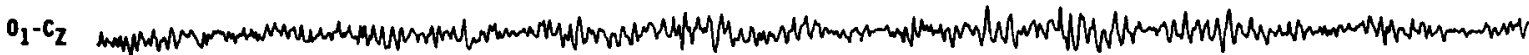

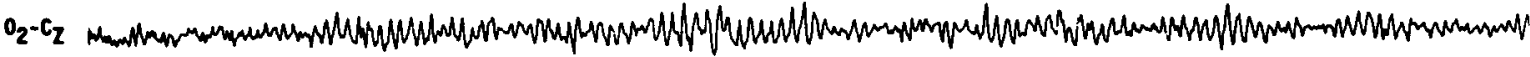

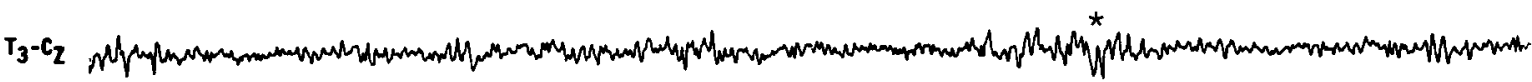

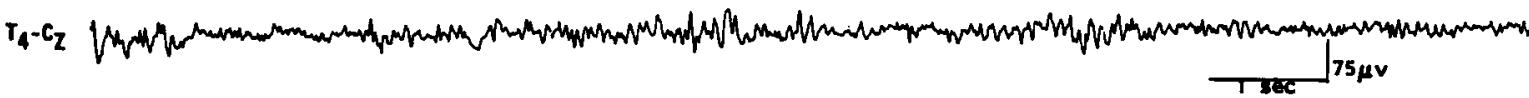

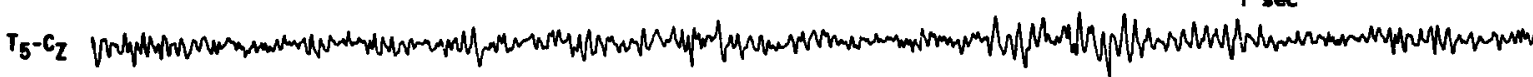

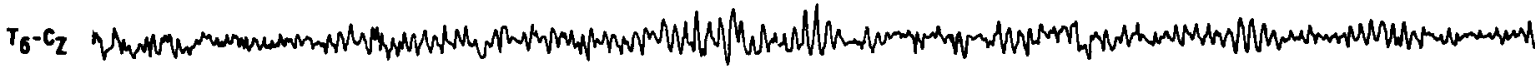

Figure 2 EEG showing intermittent slow wave transients $\left({ }^{*}\right)$ in a 61-year-old subject. 
activity in elderly subjects are likely to occur in those with intellectual impairment. ${ }^{5}$

\section{EEG IN PATIENTS WITH ALTERED MENTAL STATUS OR DIFFUSE ENCEPHALOPATHIES}

The term encephalopathy is usually applied to patients displaying altered mental status as a result of a diffuse disturbance of brain function. Common encephalopathies are divided into metabolic, toxic, inflammatory (encephalitis), anoxic, and degenerative types. The EEG in most encephalopathies shows an alteration of background activities and emergence of varying degrees of theta-delta slowing. Remember that the EEG findings are generally nonspecific from a differential standpoint. The EEG is unable to distinguish between different etiologies. The main contribution of the EEG is in providing an objective measure of severity of encephalopathy, prognosis, and effectiveness of therapy. ${ }^{10}$

There is a good correlation between the severity of the EEG changes, the severity of the encephalopathy, and the clinical state of the patient. In mild encephalopathy associated with mild clouding of consciousness and confusion, there is at first slowing of the posterior dominant rhythm, which decreases from a higher to a lower alpha frequency and then into the theta frequency range. More severe encephalopathy is associated with deeper levels of coma, and the background consists mainly of high-amplitude irregular delta activity. With further deterioration in the encephalopathy, the amplitude of all activities drop below $20 \mu \mathrm{V}$ and the EEG may consist of relatively low-amplitude, invariant delta activity. Some tracings reveal suppression-burst pattern where there is regular alternation of very-low-amplitude EEG with relatively higher-amplitude EEG segments. The most extreme type of abnormality is, of course, lack of any cerebral activity (i.e., electrocerebral inactivity). Presence of the later three types of EEG patterns (invariant low-amplitude delta, suppression-burst, and electrocerebral inactivity) carry a grave prognosis, if drug intoxication can be excluded as the cause of encephalopathy. If due to drug intoxication, these severely abnormal patterns are quite reversible with treatment, with a high potential for complete recovery of neurologic functioning.

Besides the degree of background slowing, there are two other features in the EEG that must be evaluated to determine the severity of encephalopathy. These are spontaneous variability of the EEG over several seconds to minutes, and reactivity to painful stimulation. In milder encephalopathies, the EEG shows spontaneous variability during the recording period and evidence of EEG reactivity to painful stimulation. When the EEG shows reactivity, painful stimulation commonly results in reduction of the amplitude, increase in frequency of the background activity, and reduction in the slow activity. There is often a "paradoxical activation," which is a period of more severe delta slowing following painful stimulation (Fig. 3). The presence of any type of reactivity (reduction in slow activity or increase in the degree of slowing) on painful stimulation suggests a lower grade of encephalopathy, whereas the EEG lacking spontaneous variability (invariant EEG) and total lack of any reactivity to intense and prolonged stimulation suggests a severe degree of encephalopathy.

A grading system of EEG abnormalities in adults is shown in Table 1 , similar to other rating systems. ${ }^{11,12}$ It is helpful in prognosis, evaluation of effectiveness of therapy, and comparing serial EEG studies. The slow activities associated with an encephalopathy are usually widespread and symmetrical over the two hemispheres. In children, the slowing may predominate over the posterior hemisphere, and in adults, usually over the frontal areas. These are simply maturation-related spatial EEG features, which do not signify that the encephalopathy is more severe posteriorly in children and anteriorly in adults.

It is unusual to see prominent focal or lateralized EEG findings with a diffuse encephalopathy unless there is an associated focal process, such as an old infarct or tumor. An exception is nonketotic hyperosmolar coma, a form of metabolic encephalopathy, which is very often associated with focal clinical (e.g., focal seizures) and focal EEG findings. Herpes simplex encephalitis and Creutzfeldt-Jakob disease (in the early stages) may also produce lateralized EEG slowing related to unilateral emphasis of the associated pathologic process (see below).

Another EEG pattern associated with a mild form of encephalopathy is the presence of bursts of intermittent rhythmic delta activity (IRDA) superimposed on a more or less normal background activity. Depending on the area of predominance, the IRDA is further divided into frontal or occipital types. IRDA has been traditionally considered a "projected rhythm" and a hallmark of EEG findings in patients with deep midline lesions of diencephalic, upper brain stem, or posterior fossa locations. ${ }^{13}$ Critical evaluations subsequently have cast serious doubts on this classic concept because this EEG pattern has been found in a large variety of pathological conditions and is often absent in deep midline lesions. As a matter of fact, the most common etiology of IRDA is a mild to moderate encephalopathy associated with some disturbance in consciousness (Fig. 4). ${ }^{14}$

Are there any unique or specific EEG features that help narrow the differential diagnosis of diffuse encephalopathy and point toward a more specific etiology? There are a few EEG patterns (e.g., triphasic waves, positive spikes, and periodic complexes) that, although not commonly encountered in encephalopathic $\mathrm{pa}^{-}$ tients, when present suggest a specific etiology for the encephalopathy. Periodic patterns are specifically encountered in anoxic encephalopathy and certain en- 


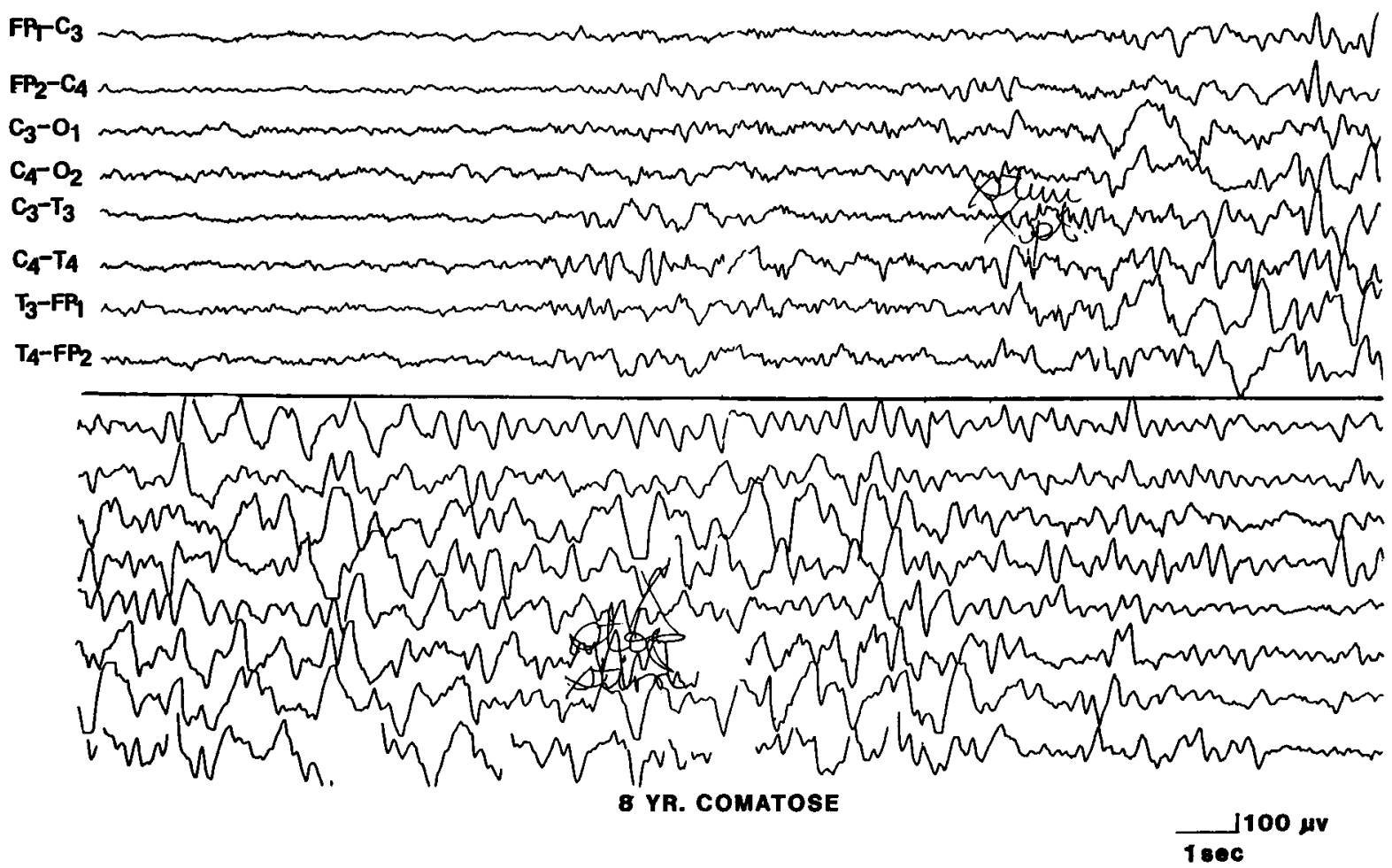

Figure 3 EEG of an 8-year-old child with hemolytic anemia and uremia, showing paradoxical activation characterized by increased delta slowing induced by painful stimulation. (Reprinted from Markand ON. Electroencephalogram in metabolic encephalopathies. Electroencephalogr Clin Neurophysiol Suppl 1999;50:301-310; with permission from Elsevier.)

cephalitides, whereas triphasic waves and positive spikes characteristically occur in metabolic encephalopathies.

\section{Metabolic Encephalopathy}

An EEG showing diffuse slowing of the background and presence of triphasic waves is highly suggestive of a metabolic encephalopathy. Triphasic waves are high amplitude (200 to $300 \mu \mathrm{V}$ ), usually bilaterally synchronous, symmetrical, and maximum in amplitude over the frontocentral regions (Fig. 5). The most prominent component is a positive sharp wave that is preceded by a short-duration negative sharp wave and followed by a long-duration negative slow wave. ${ }^{15}$ However, variations are quite common and the waveform may be monophasic or biphasic.

Although earlier authors ${ }^{15}$ emphasized that the triphasic waves were highly specific for hepatic encephalopathy, this EEG pattern has been found to correlate best with any metabolic type of encephalopathy; hepatic, renal, and anoxic etiologies account for over $75 \%$ of EEGs with triphasic waves. ${ }^{16-18}$ A feature of triphasic waves often stressed is the progressive time lag (25 to 140 milliseconds) of the positive component of the triphasic wave from the anterior to the posterior region. This feature was considered to be most specific for hepatic etiology. ${ }^{17,19}$ Recent studies ${ }^{18}$ demonstrated that the time lag is neither a consistent feature of triphasic waves, nor has any specificity with regard to the type of metabolic encephalopathy. The "peril" is that no single feature or group of features regarding triphasic waves distinguish hepatic from nonhepatic cases.

There are a few other "pearls" regarding triphasic waves. Patients with metabolic encephalopathies showing prominent triphasic wave activity in their EEG have an overall poor prognosis; in one series, over two thirds died in a matter of a few months. ${ }^{20}$ Furthermore, triphasic waves occur essentially in adults; this pattern has been rarely reported below the age of 20 years. ${ }^{21}$

Table 1 Grading of EEG Abnormalities in Diffuse Encephalopathy

\begin{tabular}{ll}
\hline Grade I (almost normal) & Dominant activity is alpha rhythm with minimal theta activity \\
Grade II (mildly abnormal) & Dominant theta background with some alpha and delta activities \\
Grade III (moderately abnormal) & Continuous delta activity predominates, little activity of faster frequencies \\
Grade IV (severely abnormal) & Low-amplitude delta activity or suppression-burst pattern \\
Grade V (extremely abnormal) & Nearly "flat" tracing or electrocerebral inactivity \\
\hline
\end{tabular}


$82 \mathrm{yr}$,

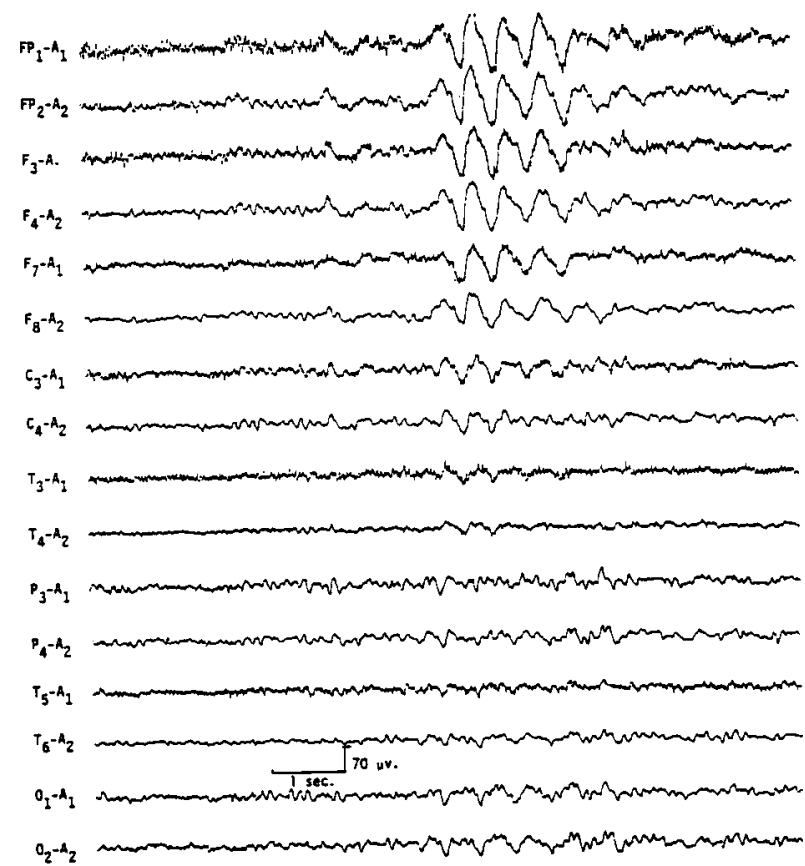

Figure 4 EEG of an 82-year-old patient with recent history of lethargy and confusion, showing frontal intermittent rhythmic delta activity. (Reprinted from Markand, ${ }^{10}$ with permission from Lippincott Williams \& Wilkins.)
This is particularly true with Reyes disease, an acute childhood encephalopathy with hepatic fatty infiltration, where triphasic waves are absent. ${ }^{22}$ The EEG pattern of 14 to 6 per second, positive spikes are a wellknown maturational EEG pattern normally seen in children in adolescence during NREM sleep. The presence of positive spike bursts in comatose patients with continuous delta activity is a unique, albeit rare, EEG pattern associated with hepatic or anoxic encephalopathy in children (Fig. 6).23,24

\section{Toxic Encephalopathy}

Overdose of hypnotic-sedative drugs is a common cause of coma encountered in the emergency room; excessive beta activity is a prominent feature in the EEG over the anterior head regions. What is less well recognized is that with more severe intoxication, the fast activity assumes a slower frequency (usually 10 to $13 \mathrm{~Hz}$ ), which is widespread but with anterior predominance. The presence of generalized theta-delta activity with superimposed alpha frequency activity is a unique encephalographic pattern highly characteristic of sedative drug intoxication (Fig. 7). In the absence of prominent slow activity, the anterior dominant generalized fast activity

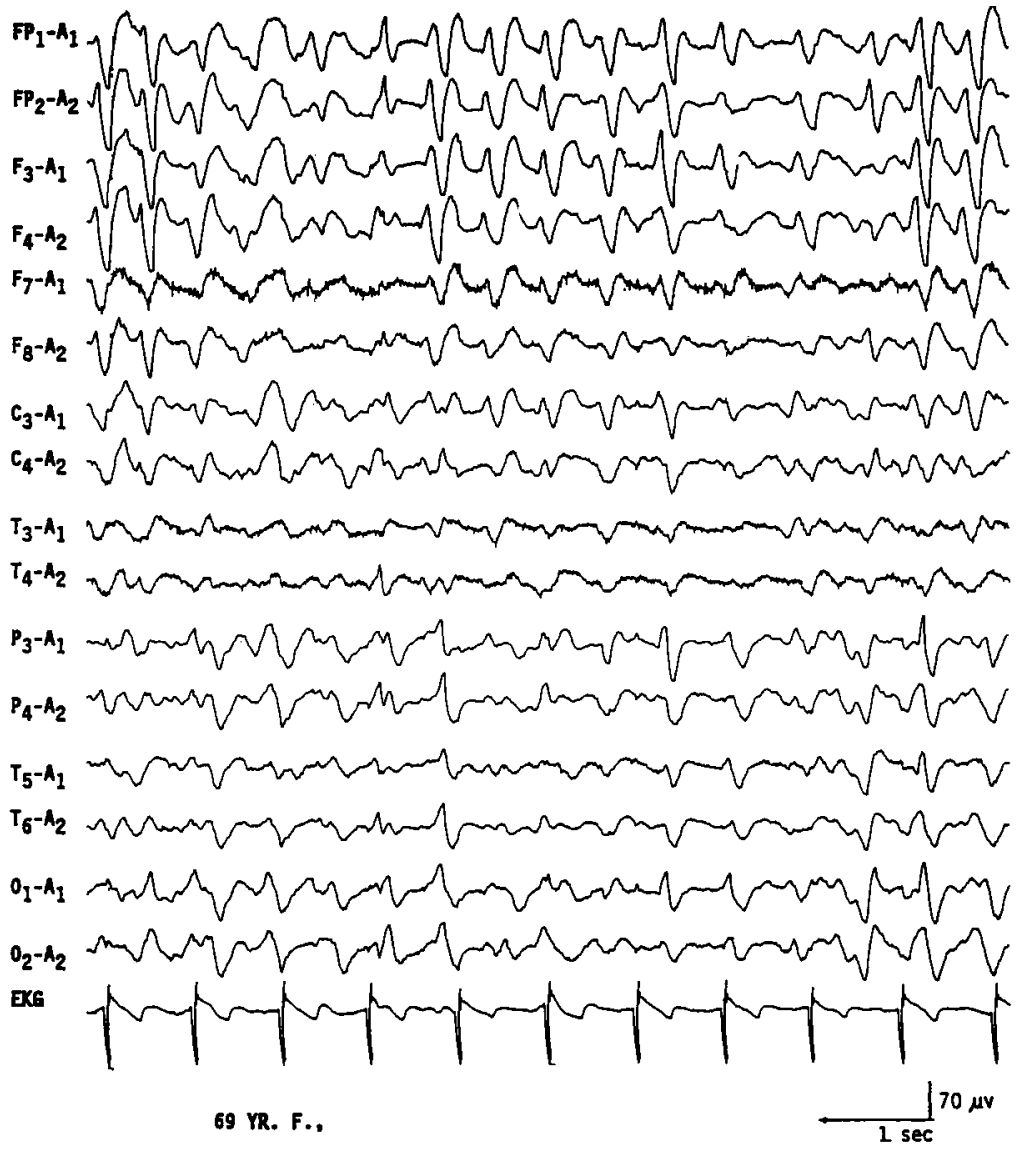

Figure 5 EEG of a 69-year-old patient with hepatic encephalopathy, showing triphasic waves. (Reprinted from Markand ON. Electroencephalogram in metabolic encephalopathies. Electroencephalogr Clin Neurophysiol Suppl 1999;50:301-310; with permission from Elsevier.) 


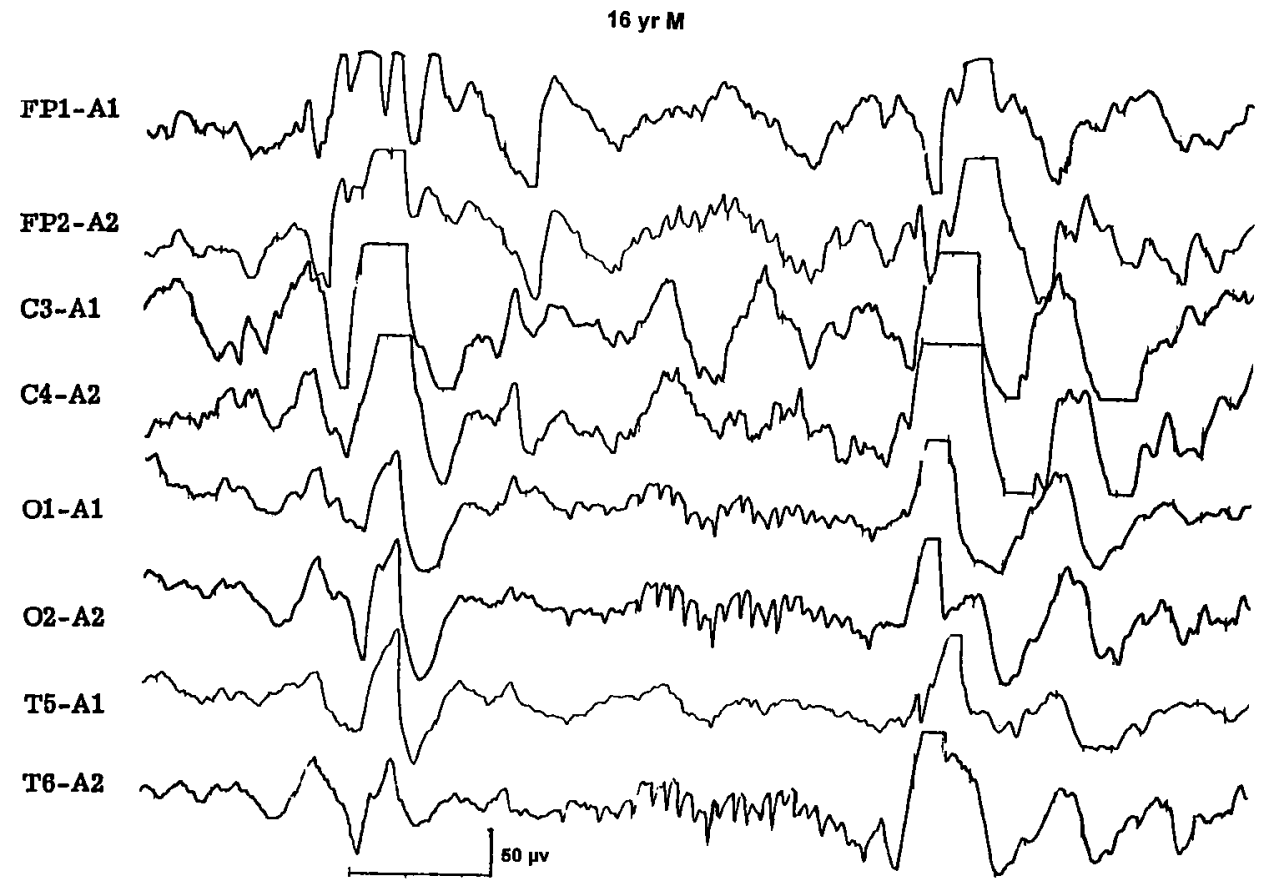

Figure 6 EEG of a 16-year-old comatose patient with Reye's syndrome, showing $14 \mathrm{cps}$ positive spikes. (Reprinted from Markand ON. Electroencephalogram in metabolic encephalopathies. Electroencephalogr Clin Neurophysiol Suppl 1999;50:301-310; with permission from Elsevier.)

produces alpha or spindle coma pattern in the EEG indistinguishable from that seen with severe anoxic encephalopathy. 25,26

Very severe drug intoxication results in suppression-burst pattern or electrocerebral inactivity. Even though these patterns signify advanced intoxication, they do not carry as ominous a prognosis as when they occur in the setting of cardiopulmonary arrest. It has been repeatedly demonstrated that patients with druginduced coma may have electrocerebral inactivity lasting over a day and may still make a full neurologic recovery. Phencyclidine hydrochloride ("angel dust," "PCP pills") is associated with a distinctive EEG pattern similar to that of subacute sclerosing panencephalitis (SSPE). The EEG shows generalized sinusoidal $6.0 \mathrm{cps}$ theta activity that is interrupted approximately every 4 seconds by generalized slow wave discharges. ${ }^{27} \mathrm{~A}$ similar periodic EEG pattern is described transiently during ketamine (a phencyclidine derivative) anesthesia.

\section{Anoxic Encephalopathy}

EEG is commonly performed in patients with anoxic encephalopathy due to cardiopulmonary arrest for assessing the severity of cerebral insult and for prognosis. Patients with normal or almost normal EEG tracings (grade I encephalopathy) following an episode of cerebral anoxia have an excellent prognosis for full neurologic recovery. On the other hand, patients with grade IV or V EEG abnormalities have a uniformly fatal prognosis; most of these patients die without regaining consciousness. An EEG should be obtained at least 5 or 6 hours after successful resuscitation since it takes an hour or more for the EEG to stabilize after an anoxic episode. ${ }^{12}$

Besides electrocerebral inactivity, there are three other unique EEG patterns, encountered in association with anoxic encephalopathy, that carry a poor prognosis for neurologic recovery.

1. Periodic discharges in anoxic encephalopathy may be either bilaterally synchronous periodic epileptiform discharges (BiPLEDs) ${ }^{28}$ or independently occurring periodic lateralized epileptiform discharges (bilateral PLEDs). ${ }^{29}$ Both periodic EEG patterns are often associated with myoclonic seizures (or even myoclonic status) and carry an extremely poor prognosis and uniform mortality (Fig. 8). Vigorous antiepileptic medication treatment of myoclonic seizures related to the two EEG patterns do not affect the ultimate prognosis.

2. Suppression-burst EEG pattern due to anoxic encephalopathy is at times associated with interesting clinical phenomena; during periods of activity both eyes may open or there are other brief body movements (Fig. 9). ${ }^{30,31}$ Whether this is an epileptic event (a brief myoclonic seizure) or a brain stem release phenomena remains unknown. At times these movements may cause confusion in the minds of relatives and even treating physicians about the patient's state 
A

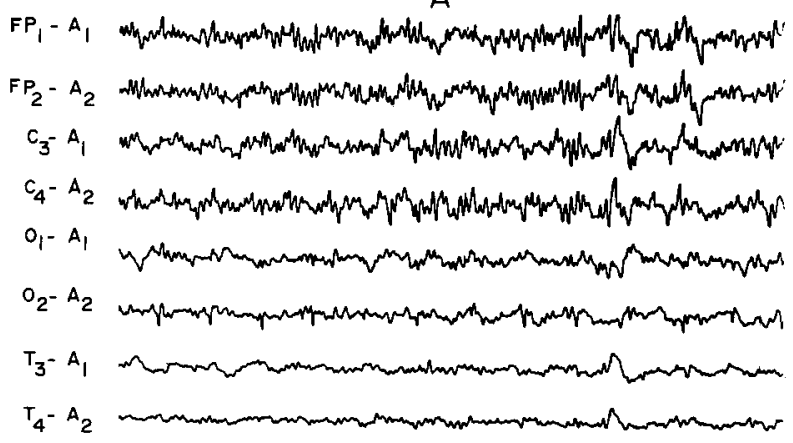

$B$

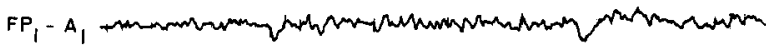

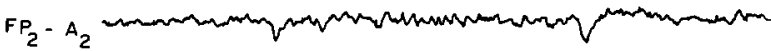

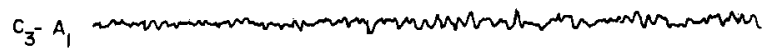

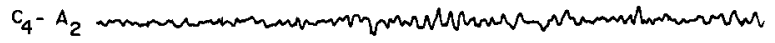

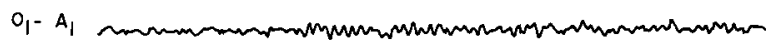

$\mathrm{O}_{2} \mathrm{~A}_{2}$ -

$T_{3} \cdot A_{1}$

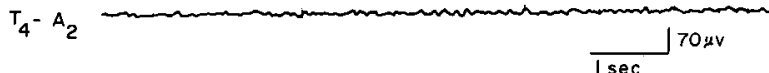

Figure 7 EEG of an 18-year-old patient with phenobarbital intoxication, showing generalized theta-delta activity with superimposed beta frequencies (A) followed in 3 days by normalization of the EEG (B). (Reprinted from Markand, ${ }^{10}$ with permission from Lippincott Williams \& Wilkins.)

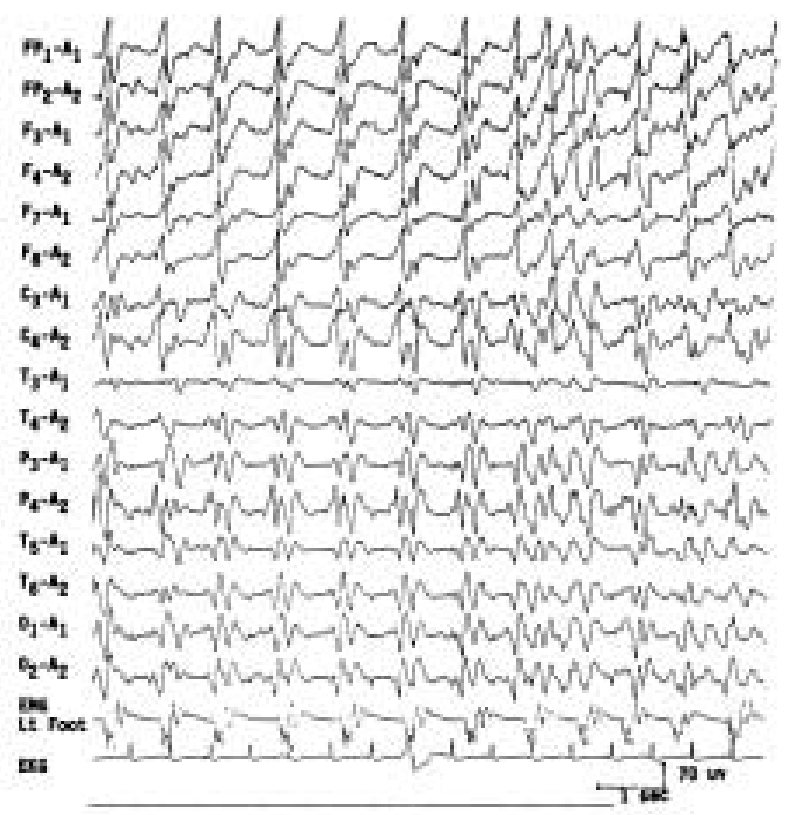

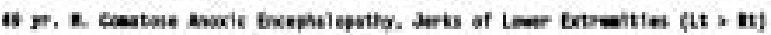

Figure 8 EEG of a 49-year-old comatose patient following severe anoxic encephalopathy, showing bisynchronous periodic epileptiform discharges synchronous with jerks of the left lower extremity monitored on a separate channel. of consciousness, as they may mimic volitional motor activity.

3. A rare EEG pattern seen in severe anoxic encephalopathy is the alpha coma pattern, denoting the conjunction of clinical coma associated with alpha frequency activity. ${ }^{32-34}$ Because in such tracings the dominant frequency is alpha frequency activity without significant slower frequencies, the EEG superficially resembles that of an "awake" person, but there are major differences. The alpha frequency activity in alpha pattern coma is widespread in distribution and is often prominent over the anterior head regions (Fig. 10). Reactivity to any type of sensory stimulation is usually absent. The prognosis of alpha pattern coma is extremely poor; all patients have either died or survived in chronic vegetative state.

Remember that EEG findings of alpha pattern coma are also seen in the setting of sedative/hypnotic drug intoxication ${ }^{25,35}$ and in association with intrinsic brain stem lesions ${ }^{36}$ with a much more favorable prognosis.

\section{Cerebral Death}

The EEG is being employed with increasing frequency for the determination of cerebral death in patients with irreversible coma, particularly when organs have to be salvaged for transplantation. It cannot be overemphasized that the absence of cerebral activity on the EEG is only one of the criteria, and should always be considered along with the clinical findings and blood flow studies for brain death. To properly identify very-low-voltage cerebral activity, to distinguish physiological or instrumental artifacts, and to eliminate the possibility of errors through malfunctioning equipment or inadequate techniques, the American EEG Society ${ }^{37}$ has a number of recommendations that must be followed during EEG recordings in all cases of suspected brain death. In such "flat" tracings, EEG activity may be obscured by verylow-amplitude fast activity due to sustained contraction of scalp muscles, which can be eliminated by giving a short-acting neuromuscular blocking agent (succinylcholine, 20 to $40 \mathrm{mg}$ IV). This step, which is very easy to undertake, is often overlooked to obtain a satisfactory recording in such patients.

A single EEG and a 6- to 12-hour clinical observation after an unequivocal acute cerebral insult are minimum requirements for brain death evaluation in an adult. In young children, the guidelines are slightly different because of the more difficult task of confirming brain death in this age group. A special task force ${ }^{38}$ rec- $^{-}$ ommended the following:

1. Brain death should not be determined until at least 7 days of age. 


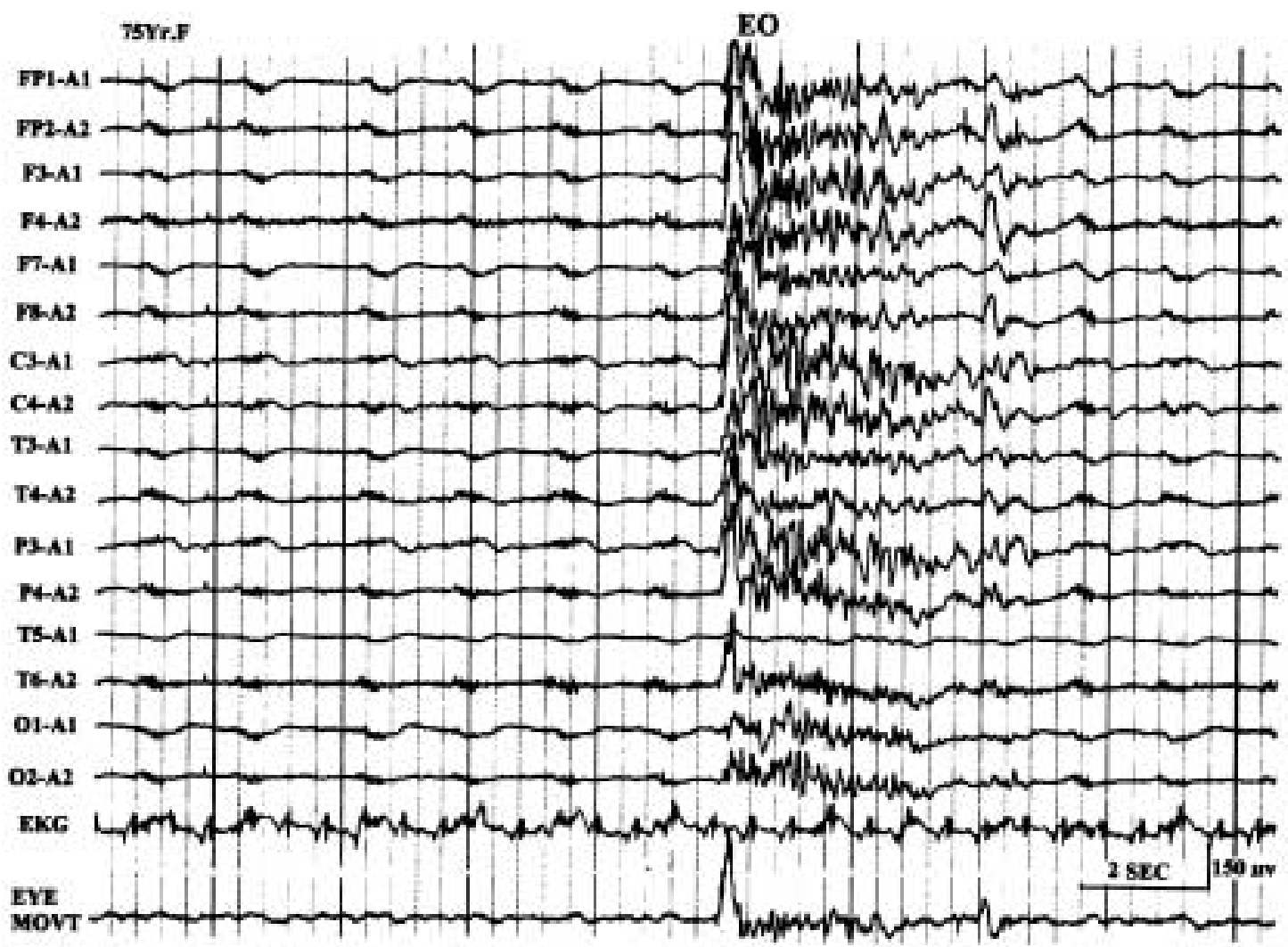

Figure 9 EEG of a 75-year-old patient with severe anoxic encephalopathy, showing suppression-burst pattern. During the burst activity there is opening of the eyes; eye movements monitored in the last channel.

2. Seven days to 2 months: two examinations and two EEGs separated by at least 48 hours are required.

3. Two months to 1 year: two examinations and two EEGs separated by at least 24 hours are required.

4. Older than 1 year: similar criteria as an adult (i.e., one EEG and at least 12 hours of observation).

\section{Encephalitides}

In viral encephalitis the severity of the EEG abnormalities generally parallel the clinical picture, but at times the EEG may be more disorganized and slow than the mental state of the patient may suggest. With a few exceptions, the EEG changes in different viral encephalitides are generally nonspecific and not helpful to distinguish one etiologic agent from another. ${ }^{39}$

The EEG pattern and its evolution in herpes simplex encephalitis are rather characteristic so that the diagnosis can often be suspected by EEG findings when considered in the proper clinical setting. The EEG in herpes simplex encephalitis may show a prominent focal abnormality, usually a focus of polymorphic delta activity over a temporal region, corresponding to the initial localization of pathology to the temporal lobe of the brain. ${ }^{40-42}$ The most characteristic EEG feature of herpes simplex encephalitis is the occurrence of pseudo-pe- riodic, focal or unilateral, large amplitude, sharp wave complexes that repeat at regular intervals of 1 to $3 \mathrm{sec}^{-}$ onds. ${ }^{40-43}$ These periodic lateralized epileptiform discharges are usually expressed maximally over the involved temporal lobe (Fig. 11). This characteristic periodic pattern is usually seen between 2 and 15 days after the onset of illness. As the disease progresses and the other hemisphere becomes involved, the periodic complexes may disappear on the side of initial involvement before appearing on the side more recently involved. With bilateral involvement of the brain, periodic complexes may occur over both hemispheres; they may then occur either synchronously or independently over the two sides.

The presence of unilateral or focal periodic complexes (PLEDs) is not unique for herpes simplex encephalitis. PLEDs may occur with acute focal cerebral hemispheric processes (e.g. infarction, brain abscess, or neoplasm). ${ }^{44}$ Nevertheless, the presence of unilateral periodic complexes in association with an acute febrile illness, focal seizures, and spinal fluid pleocytosis is strongly suggestive of herpes simplex encephalitis.

SSPE, a childhood disorder that is a slow virus infection of the central nervous system due to measles, has virtually disappeared from the United States since the introduction of measles vaccination. The EEG in 


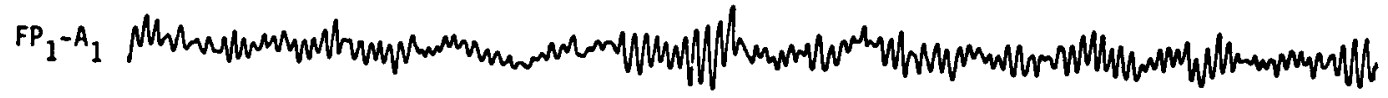

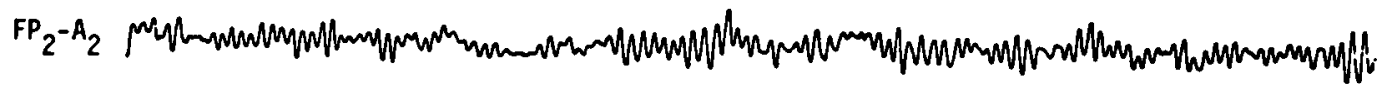

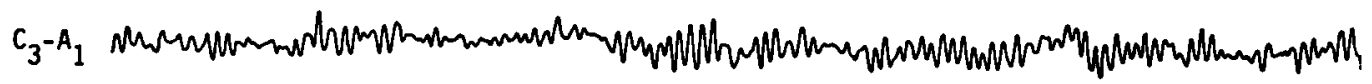

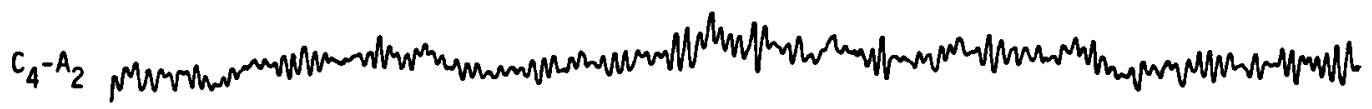

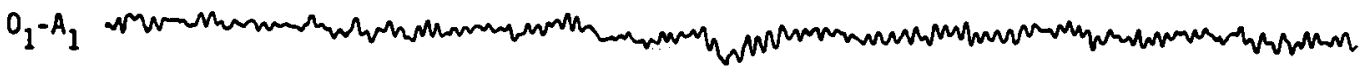

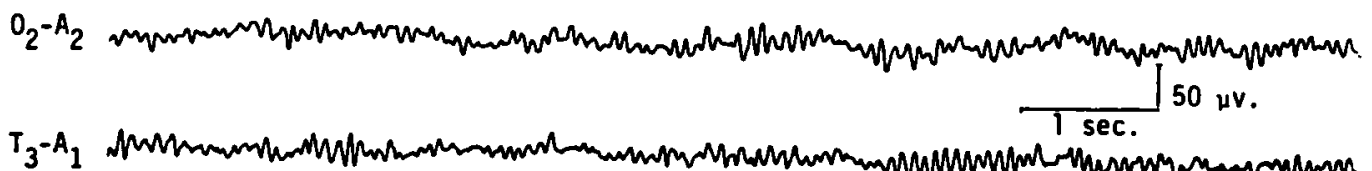

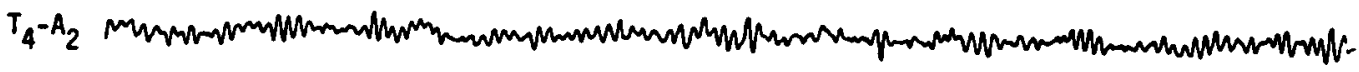

Figure 10 EEG of a 77-year-old comatose patient following cardiopulmonary arrest 4 days previously, showing "alpha coma pattern." Patient died after 2 days. (Reprinted from Markand, 10 with permission from Lippincott Williams \& Wilkins.)

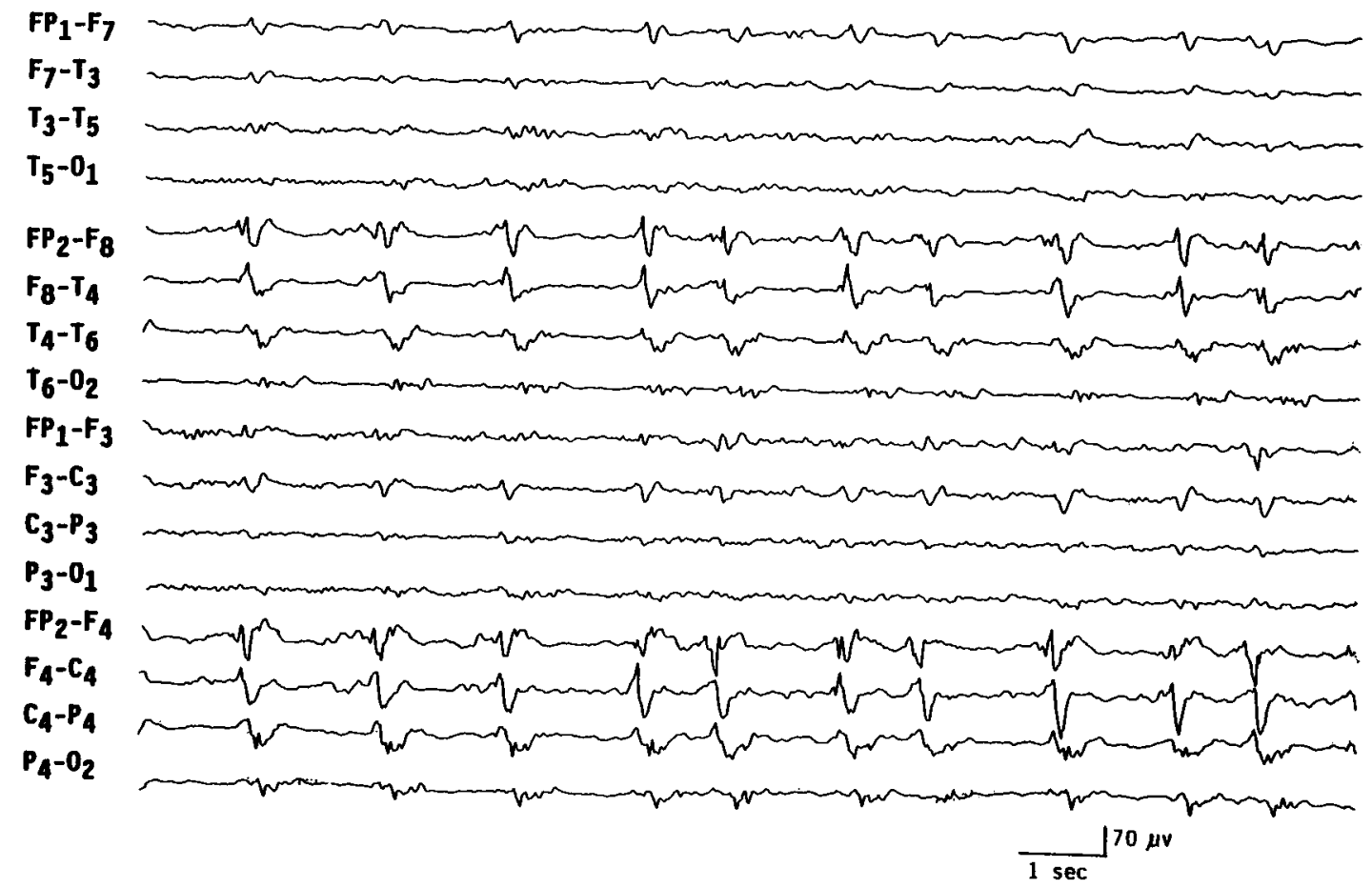

Figure 11 EEG of a 65-year-old patient with Herpes simplex encephalitis, showing periodic epileptiform discharges occurring over the right temporal region every 1 to 2 seconds. 


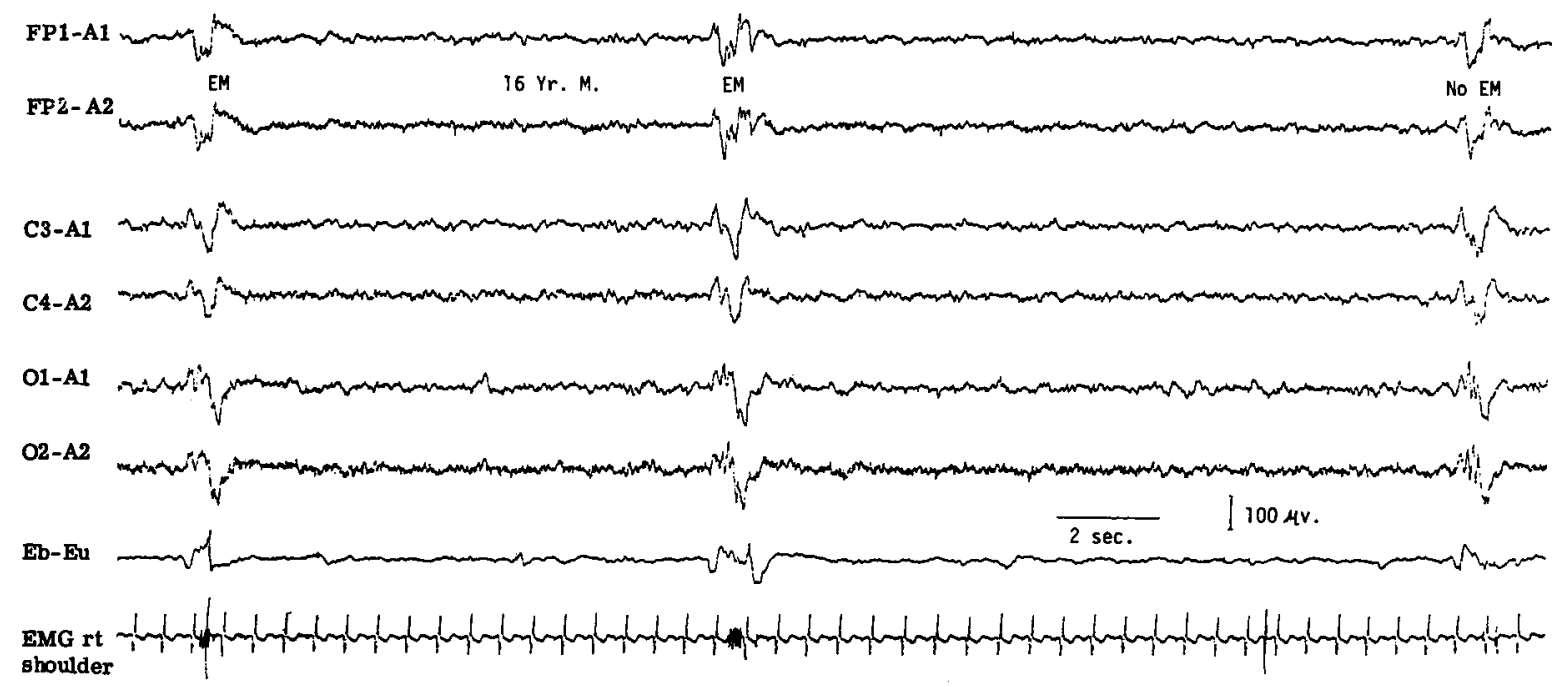

Figure 12 EEG of a 16-year-old patient with subacute sclerosing panencephalitis, showing high-amplitude generalized periodic complexes repeating at intervals of 8 to 10 seconds and accompanied by eye jerks and myoclonic jerks of the upper extremities monitored on the last two channels. (Reprinted from Markand, ${ }^{10}$ with permission from Lippincott Williams \& Wilkins.)

SSPE is highly specific and characterized by the presence of high-amplitude periodic complexes that are bilateral, usually synchronous, and symmetrical. ${ }^{45-47}$ They are remarkably stereotyped and consist of two or more delta waves with or without sharp wave components intermixed with them. The periodic complexes repeat with a fair regularity every 4 to 10 seconds and there is a 1:1 relationship of the EEG periodic complexes to the clinical myoclonic jerks, when present (Fig. 12). In the early stages of the disease, the periodic complexes may occur at irregular and long intervals, and sleep may activate them. A sleep recording, therefore, is recommended in a suspected case of SSPE in which the awake tracing has failed to reveal periodic complexes. ${ }^{48}$ Also, in the early stages there is asymmetry of the periodic complexes, which may be associated with asymmetry of the myoclonic jerks that occur contralateral to the periodic complexes. ${ }^{47}$ Later in the disease, the periodic complexes are bilaterally symmetrical and synchronous.

Creutzfeldt-Jakob disease, a prion disorder of the central nervous system (CNS), is also characterized by a very specific EEG pattern, which consists of periodic, bilaterally synchronous wave forms. ${ }^{49}$ The periodic discharges take the form of diphasic or triphasic sharp waves, which repeat regularly at a frequency close to one per second. There is a fairly close relationship between the periodic complexes and myoclonic jerks; the latter may occur a few milliseconds before or after the electrical event.

What is less well known is the fact that in the early stages of Creutzfeldt-Jakob disease, focal or lateralized periodic sharp waves (PLEDs) may occur,50,51 which later evolve into bilaterally symmetrical and synchronous periodic discharges superimposed on a "flat" background (Fig. 13). Although the periodic EEG pattern is not pathognomonic, the presence of periodic sharp waves occurring regularly around one per second, in association with clinical findings of progressive dementia and myoclonus in elderly individuals, provides strong support to the diagnosis of Creutzfeldt-Jakob disease. This characteristic periodic pattern is reported in more than $75 \%$ of patients with histologically verified Creutzfeldt-Jakob disease, and the pattern becomes fully established within the first 3 months of the onset of symptoms. . $^{52,53}$

\section{Degenerative Encephalopathies}

In degenerative encephalopathies, a common denominator is a disturbance in the regulation and frequency of the background activity, but the EEG features do differ with regard to whether the pathologic process involves predominantly cortical and/or subcortical gray matter or cerebral white matter. ${ }^{54}$ In disorders that primarily involve the cerebral white matter, the EEG is characterized predominantly by the presence of high-amplitude continuous generalized polymorphic delta activity associated with a markedly disordered background and virtual absence of epileptiform activity or paroxysmal discharges. Such changes are characteristically seen in all types of leukodystrophies, Schilder's disease, and multifocal leukoencephalopathy. In diffuse cortical gray matter encephalopathies, the EEG is characterized by abnormal background activity that is slow, irregular, and low in amplitude. There is minimal continuous generalized polymorphic delta activity, and paroxysmal findings are usually absent or minimal. Examples include 

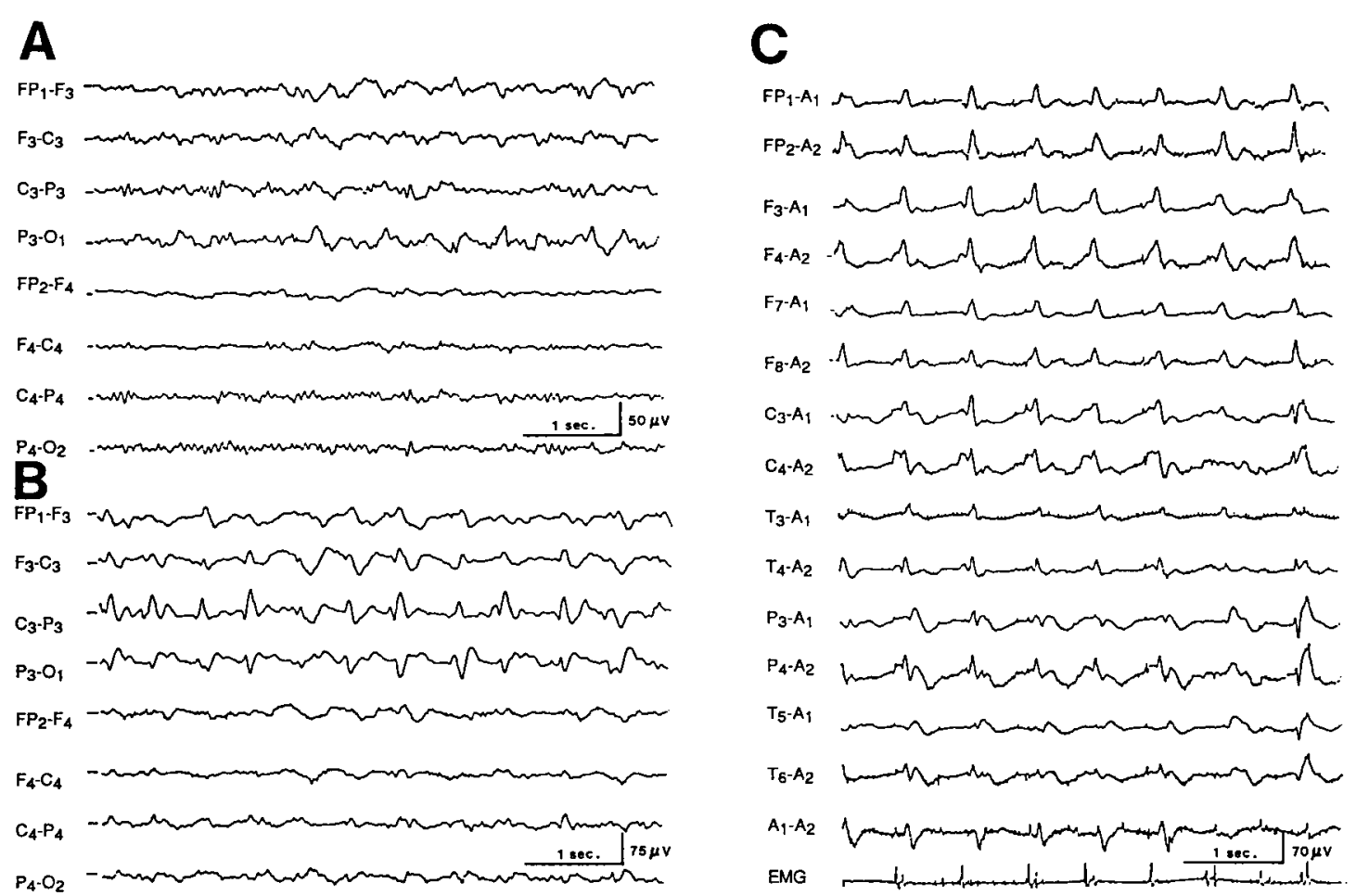

Figure 13 Serial EEGs of a 62-year-old patient with Creutzfeldt-Jakob disease. The first EEG (A), obtained 2 months after the onset of dementia and progressive right hemiparesis, shows left-sided delta activity. EEG 2 weeks later (B) shows periodic lateralized epileptiform discharges over the left hemisphere, and an EEG taken 5 months after the onset of illness (C) shows typical bisynchronous high-amplitude periodic complexes superimposed on "flat" background. Myoclonic jerks monitored on the last channel are synchronous to the periodic complexes. (Reprinted from Markand, ${ }^{10}$ with permission from Lippincott Williams \& Wilkins.)

Alzheimer's or Pick's disease. In diffuse cortical and subcortical gray matter encephalopathies, the EEG shows generalized bilaterally synchronous paroxysmal discharges in the form of bursts of monorhythmic delta waves or paroxysms of slow spike wave activity superimposed on an abnormal background. Pathological conditions include cerebromacular degeneration (e.g., Batten's disease).

Degenerative disorders with lesions predominantly below the cerebrum produce only minimal alterations in the EEG. This is usually the case in spinocerebellar degeneration, Parkinson's disease, progressive supranuclear palsy, and so on, where the EEG either remains normal or shows mild nonspecific slowing of the background activity. There are virtually no other EEG features associated with degenerative encephalopathies that have a high correlation to a specific etiologic process. The exception is the occurrence of large-amplitude spikes in response to single flashes or at flickering rates below three per second, which is a highly characteristic feature of Batten's disease..$^{55}$ These large potentials may reach 50 to $500 \mu \mathrm{V}$, maximum over the occipital region, and sometimes associated with myoclonic jerks of the limbs and face (Fig. 14). With advancing retinal disease and blindness, the characteristic photic response is lost.

In patients with senile or presenile dementia, the EEG background shows varying degrees of slowing and disorganization ${ }^{56-58}$ but may remain within normal limits in individuals with obvious intellectual impairment. A slowing of the alpha rhythm from 11 to $12 \mathrm{~Hz}$ to 8 to $9 \mathrm{~Hz}$ may represent a significant deterioration of the EEG, but in the absence of serial studies this would remain unrecognized. Furthermore, the rate of progression of dementia is important because patients with very slowly progressing dementia are likely to show minimal EEG changes. Epileptiform discharges are rare in the Alzheimer's type of presenile or senile dementia except in very advanced disease. Sharp or triphasic waves over the posterior head regions in severely demented patients have been reported. ${ }^{59}$ At times these EEG waveforms may raise a suspicion of CreutzfeldtJakob disease; however, unlike Creutzfeldt-Jakob disease, these discharges occur irregularly with little or no tendency toward periodic occurrence.

Huntington's disease has a very characteristic clinical picture, and the diagnosis is confirmed by genetic testing. There is a high incidence of abnormal EEG tracing in Huntington's disease; the characteristic feature is the presence of a "flat tracing" with virtual absence of rhythmic activity. Such features are reported in as high as two thirds of the patients with Huntington's disease. ${ }^{60}$ There is absence of any EEG activity in excess of $10 \mu \mathrm{V}$. In addition, the EEG is practically devoid of any rhythmic activity, not merely a paucity of recogniz- 


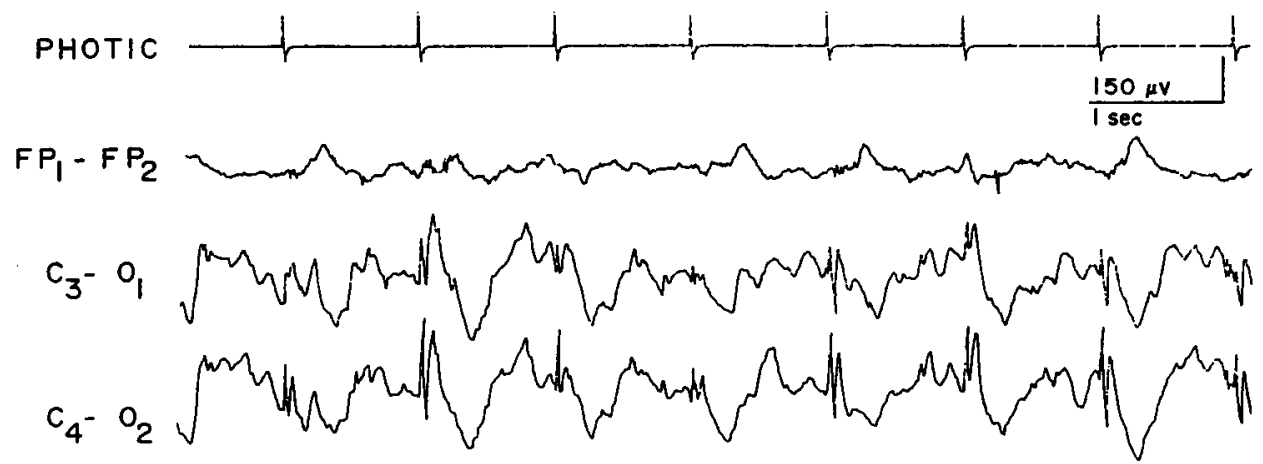

Figure 14 EEG of a 6-year-old patient with Batten's disease, showing high-amplitude spikes induced by photic stimulation at one per second. (Reprinted from Markand, ${ }^{10}$ with permission from Lippincott Williams \& Wilkins.)

able rhythms. Such "flat" EEGs in Huntington's disease need to be differentiated from a normal variant, lowamplitude tracings in adults, which have activity less than $20 \mu \mathrm{V}$ and a paucity of recognizable rhythms. Hyperventilation would increase the amount and amplitude of rhythmic activity with normal variant, whereas in patients with Huntington's disease hyperventilation remains ineffective. ${ }^{2}$

\section{EEG IN FOCAL OR LATERALIZED CEREBRAL HEMISPHERIC LESIONS}

Since the advent of computerized tomography and MRI, the EEG has been utilized less for localizing focal cerebral lesions, including brain tumors. Nevertheless, the EEG is still extensively used to evaluate the epileptogenic potential of a focal cerebral process demonstrated on imaging studies. The EEG shows focal or lateralizing findings in localized lesions that involve a superficial assessable portion of a cerebral hemisphere. ${ }^{61}$ There is slowing and decreased amplitude of the alpha rhythm on the side of the focal cerebral lesion. With extensive processes, the alpha rhythm disappears and is replaced by slower-frequency activity (theta/delta). Comparable changes can occur in the anterior beta activity and can be spontaneous or drug-induced. During NREM sleep, spindles may be less persistent and of lower amplitude as may vertex sharp transients. In massive or rapidly progressive hemispheric lesions such as a major hemispheric stroke or large glioblastoma, there may be severe depression of all EEG activities in that cerebral hemisphere.

Since Walters' observation ${ }^{62}$ a focus (localized activity) of delta activity has become the sign "par excellence" of focal structural lesions. The delta activity is called polymorphic or arrhythmic (PDA) because it consists of waves of irregular shape that change in duration, shape, and amplitude (Fig. 15) and fall in the frequency range of 0.5 to $3.0 \mathrm{~Hz}$. Focal PDA indicates a lesion that involves subcortical white matter. Greater variability in the waveform (irregularity), longer duration of waves (slower frequency), and greater persistence indicate a more severe and acute focal process. A fact less often appreciated is that in a large area of PDA the focal process is best localized to the area showing the lowest-amplitude or "flat" PDA, rather than the area showing high-amplitude PDA.63 Destructive lesions most frequently associated with focal PDA include neoplasm, abscess, infarct, hematoma, and contusion. However, focal PDA can appear transiently after a complex migraine attack or focal epileptic seizure. Hence, in a patient with prominent focal PDA with a history of a recent epileptic seizure, a repeat recording in a few days is indicated to assess the persistence or transient occurrence of this focal abnormality. Rapid disappearance of focal PDA would suggest a postictal change but would also lend support to a focal epileptic process. Static lesions such as infantile hemiplegia or Sturge-Weber syndrome $^{64}$ are associated with marked attenuation and often total absence of rhythmic activities (alpha or beta activity) over the entire affected hemisphere (Fig. 16). In contrast to progressive hemispheric lesions, such as cerebral tumor, there is very little, if any, slow activity over the involved hemisphere in such lateralized static focal processes.

Certainly, attenuation, disorganization, and slowing of the background activity on the side of the focal cerebral lesion and presence of PDA are EEG hallmarks of a focal cerebral process. Less often, the amplitude of the background activity may be higher on the side of the focal cerebral lesion, ${ }^{65}$ which may lead to an erroneous interpretation of the side of the lesion. Such increase in the amplitude of the background activity is encountered with cerebral infarcts that have "healed," with skull defect related to previous craniotomy or in patients with slowly progressive tumors (Fig. 17). Often the enhanced background activity (such as alpha rhythm) over the side of the focal cerebral process is slightly slower in frequency as well as less reactive to eye opening, ${ }^{63}$ which should alert the interpreter to the abnormality. Breach rhythms ${ }^{66}$ associated with skull defects are focal "mu-like" rhythms in Rolandic or temporal region with sporadic slow waves 


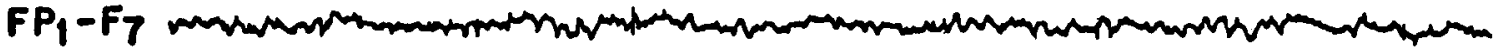

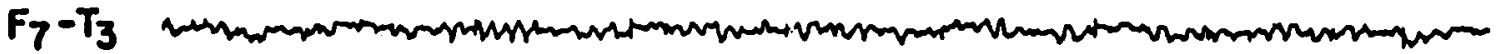

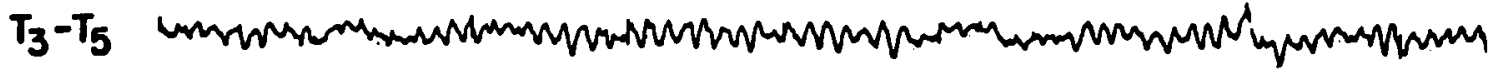

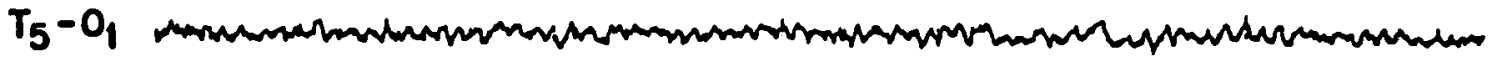

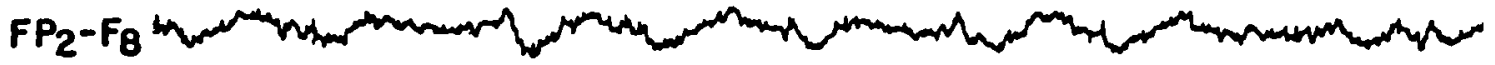

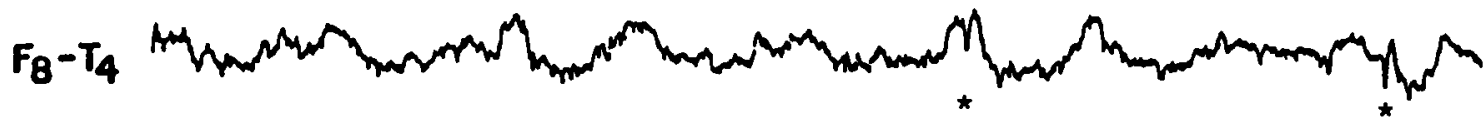

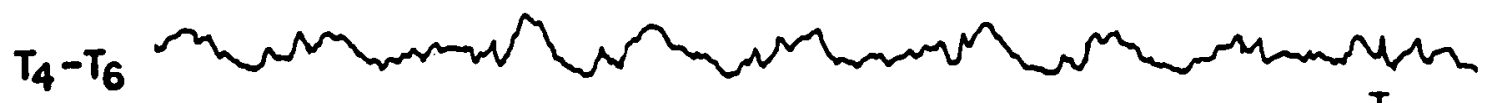
$1 \mathrm{sec}] 75 \mu \mathrm{v}$

$\mathrm{T}_{6}-\mathrm{O}_{2}$ 2

Figure 15 EEG of a 43-year-old patient with right temporal glioma, showing polymorphic delta activity and low-amplitude spike discharges $\left({ }^{*}\right)$ over the right temporal region. (Reprinted from Daly and Markand, 61 with permission from Lippincott Williams \& Wilkins.)

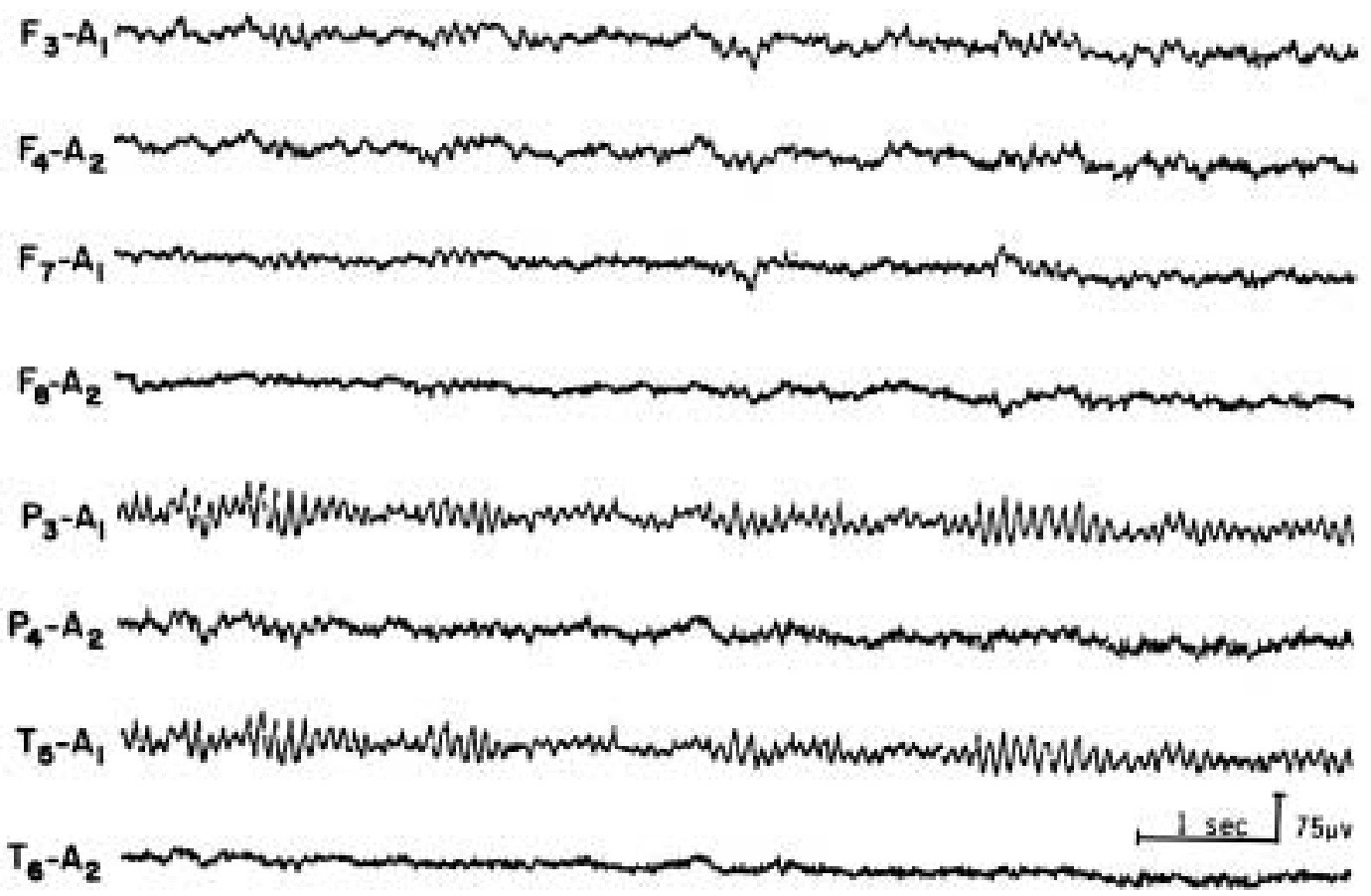

Figure 16 EEG of a 16-year-old patient with Sturge-Weber syndrome of the right hemisphere, showing total absence of rhythmic activities over the entire affected hemisphere. (Reprinted from Daly and Markand, 61 with permission from Lippincott Williams \& Wilkins.) 


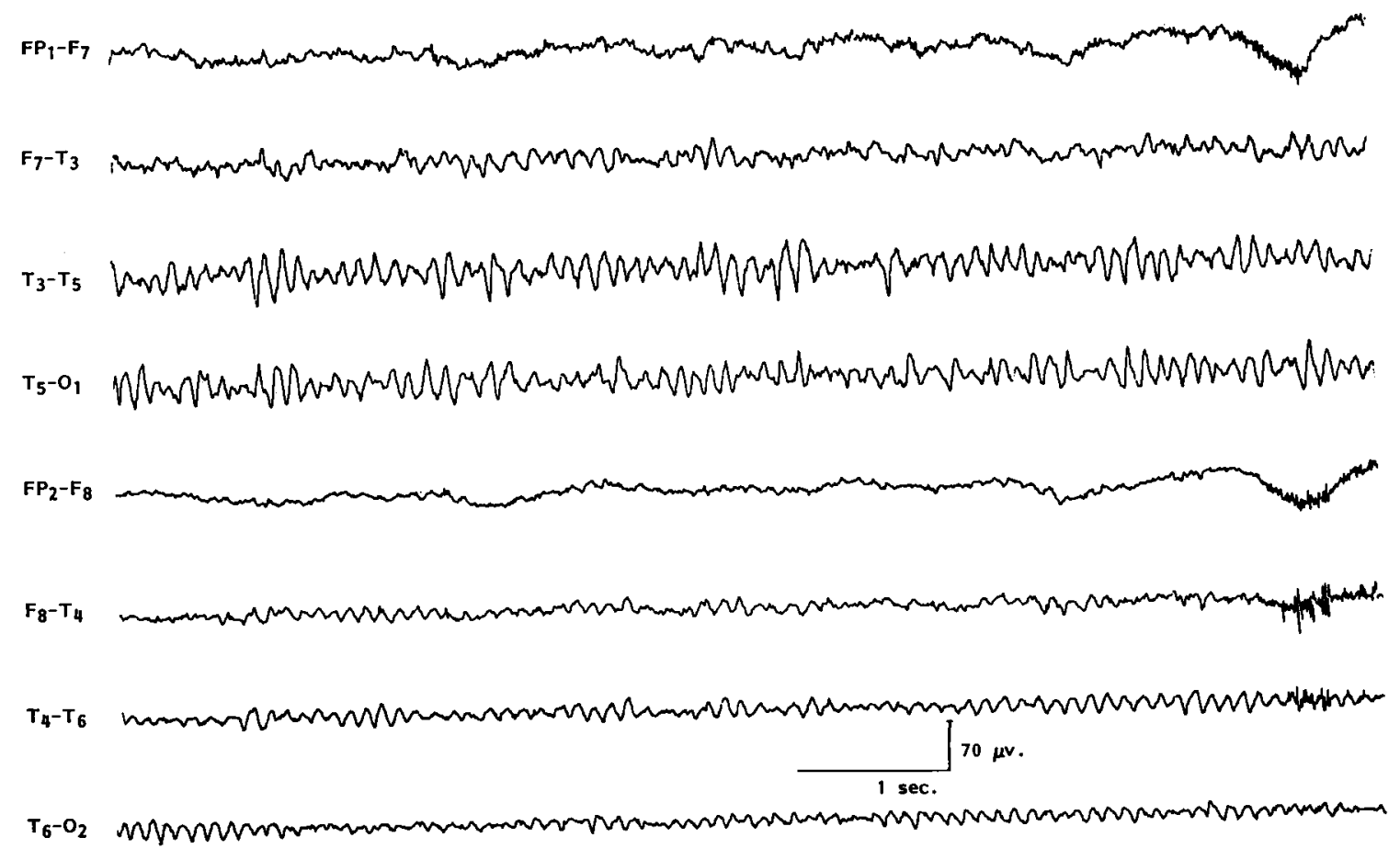

Figure 17 EEG of a 47-year-old patient with a low-grade glioma of the left temporal lobe, showing slightly slow but higher amplitude alpha on the left side.

and spiky or sharp transients (Fig. 18). These rhythms are unrelated to epilepsy and do not indicate recurrence of a tumor. The "spiky" grapho-elements should not be overinterpreted as epileptogenic discharges. For proper assessment of EEG asymmetries, it is therefore essential to know if the patient has had a craniotomy or skull defect, which may enhance background activities on the side of the breach of the skull.

Epileptiform activity, such as focal spikes, sharp waves, or spike wave discharges, also occur in localized hemispheric lesions usually of an indolent or static nature. With acute hemispheric lesions, epileptiform discharges are less common but when seen often have a periodic character. PLEDs consist of sharp waves, repeating more or less regularly at one per second over a relatively large area of the hemicranium during most of the EEG study (Fig. 19). This distinctive focal periodic pattern usually occurs in patients with acute hemispheric strokes, brain abscess, primary (usually glioblastoma) or metastatic neoplasms, and herpes simplex encephalitis. ${ }^{44,67}$

\section{EEG IN PAROXYSMAL DISORDERS}

An EEG is the most common and most useful test performed in evaluating patients suspected of epilepsy. There are many areas where an EEG has unique contributions. The value of an EEG lies in the fact that it not only shows specific ictal discharges during a clinical seizure but also characteristic epileptiform abnormalities in a high proportion of epileptic patients even in the interictal period. Furthermore, an EEG may be the only test demonstrating focal abnormalities responsible for the patient's epileptic seizures. Specific patterns in the EEG make it possible to classify the seizure type, which is an essential prerequisite to institute proper antiepileptic medication. An EEG is indispensable for the diagnosis of nonconvulsive epileptic status presenting as prolonged "twilight" state or a prolonged episode of abnormal behavior. In a patient with bizarre motor activity, the recording of an EEG during such an episode may be the only way to establish whether the abnormal behavior is due to an epileptic seizure or a nonepileptic event, physiologic or nonphysiologic. Finally, the EEG is indispensable to localize the epileptogenic (seizure producing) zone before resective surgery (excision of the epileptogenic zone) is undertaken in a patient with medically refractory focal epilepsy.

\section{Epileptiform Abnormalities}

Paroxysmal EEG activities, whether focal or generalized, are often termed "epileptiform activities." They are the EEG hallmark of epilepsy as they are highly correlated with the occurrence of clinical seizures. Epileptiform abnormalities are usually divided into "interictal" discharges, which appear in the interval between clinical seizures, and "ictal" discharges, which accompany 


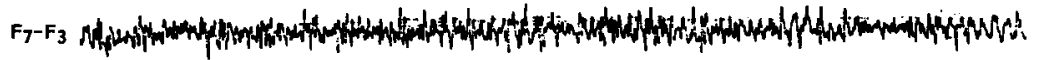

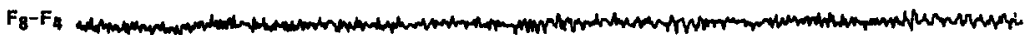

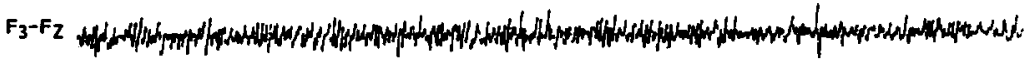
$F_{\mathbf{4}}-F_{\mathbf{Z}}$

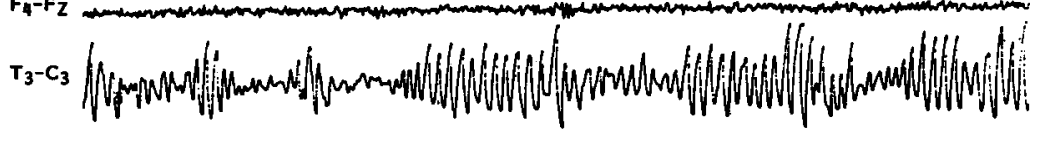

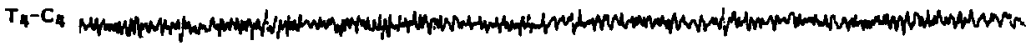

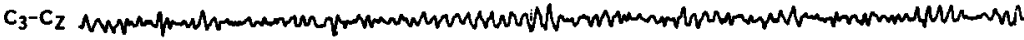

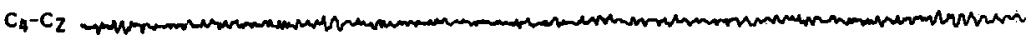

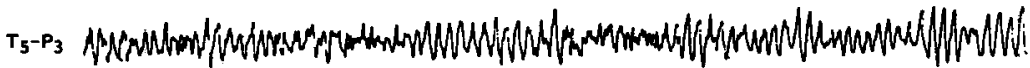

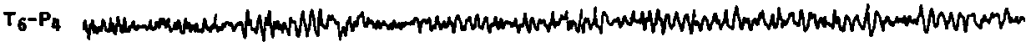

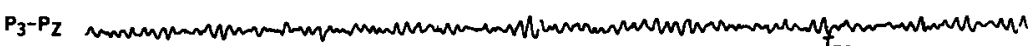
1 sec. $70 \mu \mathrm{v}$

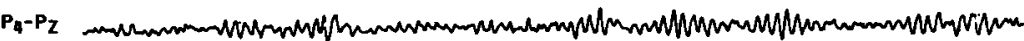

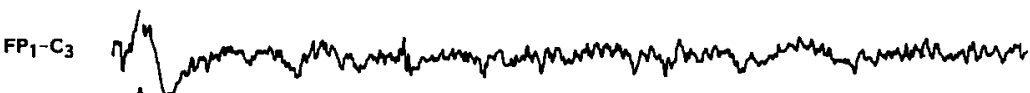

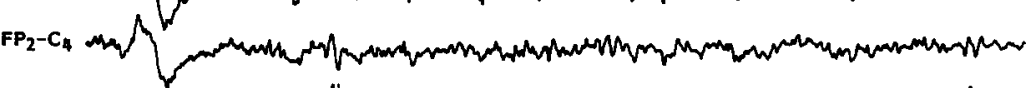

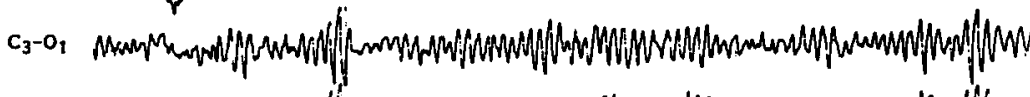

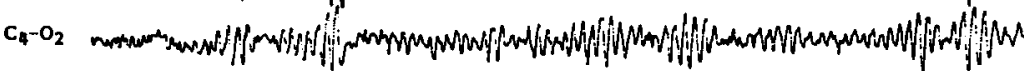

Figure 18 EEG of a 47-year-old patient with history of previous left craniotomy, showing breach rhythm in the left temporocentral region.

62 Yr.

FP1-F7

F7-T3

T3-T5

T501

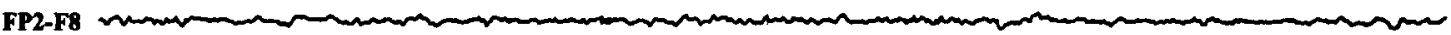

F8-T4

T4-T6

T6-02

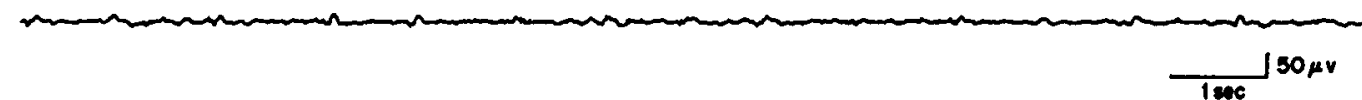

Figure 19 EEG of a 62-year-old patient with acute onset of right hemiparesis, aphasia, and right focal motor seizures, showing periodic lateralized epileptiform discharges over the right posterior temporal region. 
clinical seizures. The distinction is arbitrary because the designation "ictal" or "interictal" often depends on how closely the patient was clinically observed because minimal behavioral alterations associated with the EEG paroxysms can be easily missed. Morphologically, interictal epileptiform abnormalities consist of spikes and polyspikes, sharp waves, spike-slow wave complexes, multiple (poly) spike wave complexes, and sharp-slow wave complexes. Spike is defined as an EEG transient clearly distinguished from the background, with a pointed peak at conventional paper speed and a duration of 20 to 70 milliseconds. Sharp waves are transients of similar character as spikes but have a duration of longer than 70 milliseconds and less than 200 milliseconds. Spike-slow waves and sharp-slow wave complexes are constituted by spikes or sharp waves followed by a high-amplitude slow wave.

Morphologic characteristics of epileptiform discharges have little correlation with different types of epileptic seizures. The topographic distribution of these discharges are more important in the classification of epilepsies. Generalized discharges that are bilaterally synchronous and symmetrical are associated with generalized epilepsies, whereas focal or lateralized discharges constitute the EEG "signature" of partial (focal) epilepsies. Most patients do not have their epileptic seizures during the brief period of routine EEG; hence, the interictal epileptiform abnormalities are the ones heavily relied on for the diagnosis of epilepsy. Although the interictal epileptiform abnormalities have a high correlation with the occurrence of clinical seizures, they do not themselves mean that the patient has epilepsy. The irrefutable evidence of epileptic seizure is a clinical seizure associated simultaneously with ictal discharges in the EEG, although such evidence is often difficult to obtain except during prolonged video EEG monitoring.

"Ictal" or an electrographic seizure pattern is characterized by repetitive EEG discharges with relatively abrupt onset and termination, and characteristic pattern of evolution lasting at least several seconds. The commonest waves or complexes vary in form, frequency, and topography. The ictal pattern is generally rhythmic and frequently displays increasing amplitude, decreasing frequency, and spatial spread during the seizure. The three EEG characteristics of a focal "ictal" pattern, therefore, consist of sudden onset/termination, occurrence of a rhythmic pattern of activity during the epileptic seizure, and its characteristic evolution with respect to amplitude, frequency, and spatial distribution.

\section{Proper Identification of Diagnostic Epileptiform Abnormalities in the EEG}

In the evaluation of abnormalities in the EEG, one needs to be constantly aware that there are many EEG transients that morphologically resemble epi- leptiform discharges and that need to be distinguished from diagnostically crucial epileptiform abnormalities to avoid overdiagnosis or misdiagnosis. These include:

1. Artifacts: for example, electrode pop, muscle potentials, eye movements, electrocardiogram (EKG), etc.

2. Normal components of ongoing background activity: for example, vertex sharp transients of sleep, POSTs, mu rhythm, lambda waves, drowsy activity during sleep in children that may often be associated with sharp components, etc.

3. Epileptiform variants of dubious clinical significance: there are a large number of benign epileptiform variants that must be recognized, lest they be misinterpreted. Although morphologically similar, they are nonepileptogenic as they have no established relationship with the process responsible for generating epileptic seizures. Such sharp transients include 14 to 6 per second positive spikes, small sharp spikes or benign epileptiform transients of sleep, $6 \mathrm{~Hz}$ spike wave or phantom spike wave, wicket spikes, psychomotor variant pattern or rhythmic midtemporal discharges, breach rhythm, etc. Sleep not only activates diagnostically useful epileptiform EEG patterns, but also unmasks several types of nonepileptogenic sharp transients.

It is critical that the EEG interpreter has clear criteria for distinguishing diagnostically relevant epileptiform discharges from sharply contoured background activity or benign variants. Useful criteria have been formulated for identification of epileptiform events $^{68,69}$ :

1. Epileptiform discharges (spikes, sharp waves, and spike wave complexes) should be unarguably discrete events, not just accentuation of part of an ongoing sequence of waves. They should be clearly separable from ongoing background activity, not only by their higher amplitude but also by their morphology and duration.

2. Most epileptiform discharges have a bi- or triphasic waveform and they have a more complex morphology than even high-voltage background rhythms.

3. The epileptiform events are not sinusoidal but rather show asymmetric, rising and falling phases.

4. Most spikes and sharp waves are followed by a slow wave.

5. Finally, they should have a physiological potential field involving more than one electrode that helps to distinguish them from electrode-related artifacts or muscle potentials. 


\section{Specificity of Interictal Epileptiform Abnormalities}

Are "hard-core" epileptiform abnormalities encountered in normal children and adults who do not have a history of epileptic seizures? Different studies, some in children ${ }^{70,71}$ and others in all age groups, ${ }^{72-74}$ found an incidence of less than 2 to $4 \%$ of epileptiform abnormalities in the EEG of nonepileptic subjects. In an interesting study on EEG findings in 13,658 males ages 17 to 25 without a previous history of significant illness who were medically screened for training in the Royal Air Force of England, 69 (0.5\%) had unequivocal epileptiform discharges. ${ }^{75}$ Hence, the incidence of epileptiform abnormalities in the healthy population was significantly lower than the 2 to $4 \%$ noted in the nonepileptic patients referred to hospital EEG laboratories.

One can certainly conclude that if an individual has a "blackout spell" or episodic loss of consciousness, it is very likely to be an epileptic seizure if there are unequivocal epileptiform discharges recorded in the EEG. To reemphasize, interictal epileptiform discharges in the EEG are never diagnostic of epilepsy by themselves, but in the appropriate clinical setting, they provide important circumstantial evidence for the diagnosis of epilepsy.

\section{Sensitivity of EEG and Techniques to Improve the Yield of Interictal and Ictal EEG Abnormalities in Patients with Epileptic Disorders}

Some patients with unequivocal epilepsy, especially focal epilepsy, may have repeatedly normal or nonspecific EEG studies. A single routine EEG consisting of half an hour recording during wakefulness, hyperventilation, and intermittent photic stimulation (IPS) provides diagnostic findings in approximately half of the patients with epilepsy.76 The following describes a few ways to increase the yield of epileptiform abnormalities in an interictal EEG study.

\section{SERIAL EEG STUDIES}

EEGs recorded on more than one occasion will increase the chance for recording a specific epileptiform abnormality. Research ${ }^{76}$ has demonstrated that serial EEG studies increase the yield for epileptiform abnormalities from $50 \%$ in the first record to $84 \%$ by the third EEG, and in $92 \%$ by the fourth EEG. There was little additional yield to serial EEGs beyond this point. ${ }^{76}$ Thus, four or five EEG studies spread over a few years provide diagnostic abnormalities in over $90 \%$ patients with epilepsy. An opposite corollary is also true; serial nega- tive EEG studies in a patient with continuing paroxysmal events should raise suspicion of nonepileptic episodes. It is also well known that interictal epileptiform discharges markedly increase after a clinical seizure ${ }^{77}$; hence, obtaining an EEG promptly after a clinical seizure will increase the chances of capturing interictal epileptiform discharges.

\section{ACTIVATING PROCEDURES}

Activating procedures (e.g., hyperventilation, IPS), recording during sleep, are very well known to activate epileptiform discharges not recorded in the awake tracing. Hyperventilation and IPS are potent activators of generalized spike wave discharges associated with primary generalized epilepsies. On the other hand, sleep tends to bring out focal epileptiform abnormalities in patients experiencing focal epileptic seizures. Sleep activates virtually all focal epileptiform abnormalities; therefore, every patient suspected of epilepsy should have a sleep recording unless there is an unequivocal and specific abnormality displayed optimally during wakefulness. One of the best ways to ensure a sleep EEG is to instruct the patient to come for the EEG test after remaining awake during the entire or at least a major part of the previous night. Sleep deprivation appears to have a further activating effect that is additive to natural sleep itself, particularly in patients with complex partial seizures and in patients with juvenile myoclonic epilepsy.

Normal response to IPS includes photic driving (photic following) at flash rate or at harmonics. In about $5 \%$ of patients, asymmetric photic driving response ( $>50 \%$ difference in amplitude) may occur, which by itself (without asymmetric awake and/or sleep activities) has no clinical significance. IPS is especially helpful in patients with primary generalized epilepsy in eliciting abnormal paroxysmal discharges. Photoparoxysmal response (PPR) has a high correlation with clinical epilepsy. It is characterized by the occurrence of generalized bilaterally synchronous spike wave or multiple spike wave discharges occurring with IPS. The most effective frequency is around 15 flashes per second but other frequencies may be equally effective. Reilly and Peters distinguished two types of PPR: prolonged (selfsustained), which continues for a short period after the stimulus has been withdrawn (Fig. 20), and self-limited, which cease before the flashes stop. ${ }^{78}$ There is a much higher incidence of epilepsy in patients with prolonged (93\%) compared with the self-limited (52\%) PPR. A 1992 meta-analysis of the studies on PPR concluded: (1) PPR, prolonged or self-limited, had a significantly higher incidence of seizures than controls; (2) a prolonged PPR was associated with a much higher incidence of seizures (85\%) than the self-limited group 


\section{Yr. M.}

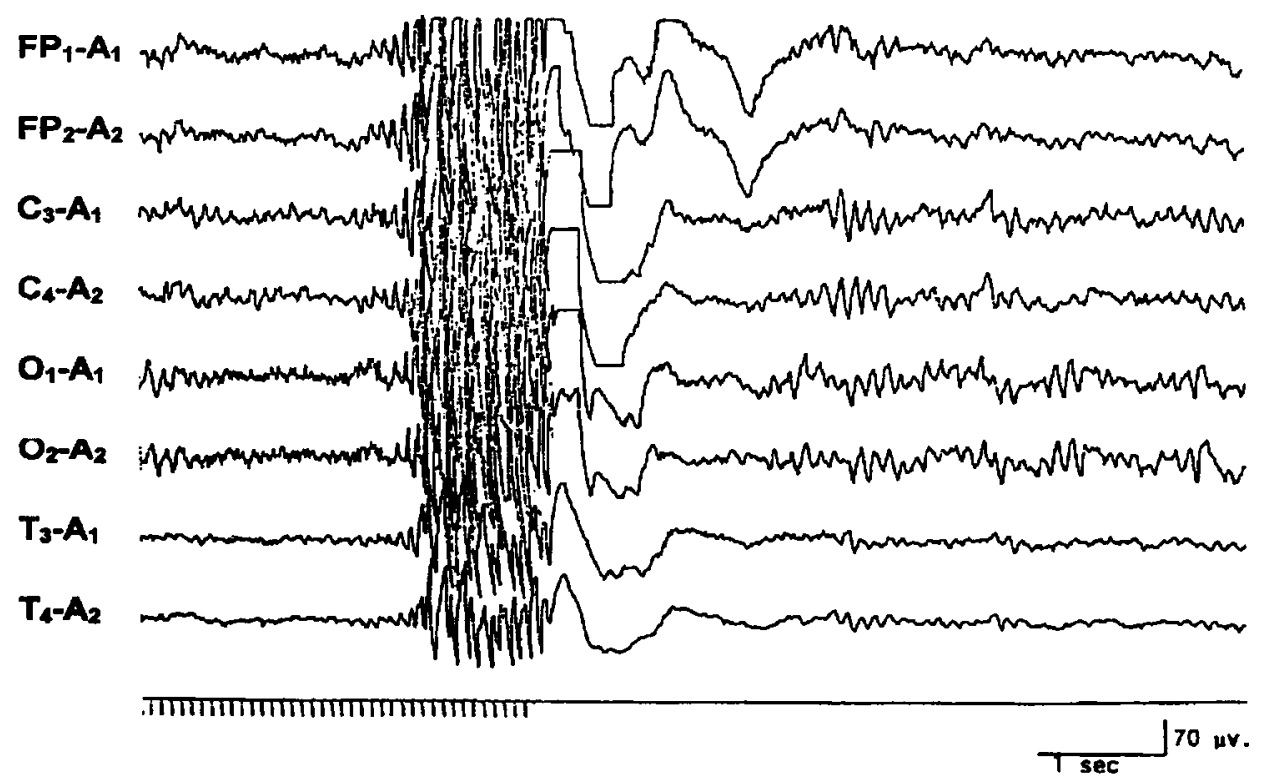

Figure 20 An EEG of a 15-year-old patient with primary generalized epilepsy, showing prolonged (self-sustained) photoparoxysmal response.

(50\%); (3) patients with prolonged PPR more often had other epileptiform abnormalities in their resting EEG than the self-limited group; (4) the risk of epilepsy increased if the PPR was associated with epileptiform abnormalities in the resting EEG; (5) the seizure incidence associated with self-limited PPR without other epileptiform abnormalities was lower (30\%) but still significantly higher than patients without PPR. ${ }^{79}$

Another photic-induced response that may superficially resemble PPR but has no significant correlation with epilepsy is a photo-myoclonic response (PMR). It consists of frontally dominant polyspikes synchronous with the flash rate and accompanied by rhythmic jerking of the muscles of the forehead, eyelids, and face. The response is blocked when the eyes are opened and it promptly stops with withdrawal of photic stimulation. PMR is generally considered to be a muscular response without cerebral participation but some regard it to be an expression of cortical response within the spectrum of photic cortical reflex myoclonus. It is seen in some nervous or tense individuals or in patients with psychiatric troubles or elderly subjects. Its presence in an individual case has no diagnostic significance.

Besides photic driving, PPR, and PMR, other less common IPS-induced EEG responses include:

1. Posterior hemispheric stimulus-dependent response: an anomalous steady state flash visual evoked potential (VEP) of unusually sharp waveform or high am- plitude. This has no clinical correlation except when it represents very-high-amplitude and spiky VEPs in association with Batten's disease or neuronal ceroid lipofuscinosis.

2. Rarely, IPS may activate a focal epileptiform discharge, usually an occipital spike focus.

3. Less often IPS may induce a frank seizure with clinical correlates (e.g., an absence, absence with eyelid myoclonus, single random generalized myoclonic jerks, repeated myoclonic jerks, occipital onset focal seizure, and rarely even a generalized tonic-clonic seizure).

Remember than $10 \%$ of patients with all forms of primary generalized epilepsy show PPR, with the highest incidence (30 to $35 \%$ ) in juvenile myoclonic epilepsy. ${ }^{80}$ The incidence of PPR is about $15 \%$ in childhood absence epilepsy, $<10 \%$ in juvenile absence epilepsy, and 10 to $15 \%$ in epilepsy with generalized tonic-clonic seizures on awakening. PPR is also common in infantile and childhood epilepsies with myoclonic seizures such as benign and severe myoclonic epilepsies of infancy and myoclonic-astatic epilepsy of childhood. PPR is rare in focal epilepsies and secondary generalized epilepsies (e.g., Lennox-Gastaut syndrome).

It must be emphasized that PPR can be detected, although rarely, among individuals with headaches or other complaints, during evaluation for aviation jobs, or as a genetic marker in a susceptible individual with a family history of idiopathic generalized epilepsy. PPR 
in nonepileptic subjects has a prevalence of 1 to $4 \%$; the response then is usually brief and less prominent.

IPS is not a totally benign activating procedure. One can induce a generalized tonic-clonic convulsion (often the first one) if photic stimulation is continued over a long duration in a patient who shows prominent PPR. It is recommended that the photic stimulation be limited to short periods ( 1 to 5 seconds) and terminated promptly as soon as generalized spike wave activity is recorded (Fig. 20).

\section{SPECIAL ELECTRODES}

Although the standard 10 to 20 international system of electrode placement provides reasonable coverage of the whole head, certain areas that have high epileptogenicity, such as the mesial temporal lobes in patients with mesial temporal sclerosis, are not fully explored by conventional placement and may require additional electrodes. Nasopharyngeal electrodes have been widely used in the past in patients suspected to have with temporal lobe epilepsy. They are associated with variable degrees of discomfort and may prevent the patient from attaining sleep during the EEG recording. They have now been largely replaced by the use of anterior temporal electrodes, which are placed $1 \mathrm{~cm}$ above and one third the distance along the line from the external auditory meatus to the external canthus of the eye. ${ }^{81}$

In a comparison of the percentages of spikes detected by standard scalp electrodes, anterior temporal, mini-sphenoidal, surface sphenoidal, and nasopharyngeal electrodes in patients with suspected complex partial seizures, the anterior temporal electrodes provided significant improvement in detecting epileptiform abnormalities. ${ }^{82}$ Recordings from standard scalp electrodes detected $58 \%$ of the discharges. Anterior temporal electrodes were the best; they detected $70 \%$ of all the discharges by themselves, and $81 \%$ in combination with standard scalp electrodes. It can be concluded that recordings from anterior temporal electrodes must be done to improve the detection of interictal epileptiform abnormalities in patients suspected of having temporal lobe epilepsy. Sphenoidal electrodes are almost invariably employed during video EEG monitoring as a part of the presurgical evaluation of patients with medically intractable complex partial seizures. The yield of abnormality from sphenoidal recordings is certainly greater than that with nasopharyngeal or anterior temporal electrodes, but it is difficult to justify the use of invasive electrode placement in routine EEG study for paroxysmal disorders.

\section{ACTIVATION OF AN ACTUAL SEIZURE DURING ROUTINE EEG STUDY}

All efforts must be made to capture the patient's habitual episode during a routine EEG. If precipitating factors are known, these are appropriately exploited. $\mathrm{Hy}^{-}$ perventilation, a potent precipitator of an absence seizure in a child with primary generalized epilepsy, must always be utilized for at least 5 minutes, once in the beginning and again at the end of a routine EEG study. In rare patients with reflex epilepsy, playing specific music in musicogenic epilepsy, asking a patient to read from a book in reading epilepsy, bathing the patient in bathing epilepsy, asking the patient to eat his meals (eating epilepsy), smelling gasoline, and so on, may all be carried out to promote an ictal event.

In patients with possible pseudoseizures, suggestion protocols are often useful in precipitating episodes and demonstrating EEG changes. ${ }^{83}$ It is important to emphasize that induced seizures must be clinically typical of a patient's habitual episodes before the diagnosis of pseudoseizures is strongly considered.

\section{Some Interpretive Challenges of EEG Findings During Paroxysmal Events}

Some of the pitfalls regarding ictal EEG changes during an actual seizure need to be stressed. All epileptic seizures are not associated with distinctive concomitant surface EEG changes. Seizures that remain very localized, including epilepsia partialis continua and simple partial seizures (focal seizures with preserved consciousness), may not have changes in the scalp EEG because the diagnostic discharge may be deepseated or involve only a small pool of neuronal tissue. However, epileptic seizures manifested by loss of consciousness, on the other hand, are accompanied by demonstrable changes in the scalp EEG. Therefore, absence of such changes during a clinical episode of "unconsciousness" or bilateral widespread motor activity (resembling grand mal seizure) can be particularly important in making the diagnosis of nonepileptic events or pseudoseizures. Ten to $20 \%$ of patients with pseudoseizures do have epileptic seizures as well. The most one can say is that at least some of the clinical episodes appear to be functional, and this must be considered within the context of the entire clinical picture.

In evaluating patients with muscle jerks or other brief motor events, it needs to be established whether these represent epileptic phenomena. Simultaneous recording by placing two electrodes over the involved muscle may be very helpful in establishing the relationship of, or lack of, the EEG and the motor recordings. In patients with myoclonic seizures, it is not always easy to establish whether an electrical event synchronous with the motor jerk is indeed a cerebral discharge or simply a movement artifact. The presence of morphologically similar EEG discharges in other portions of the tracing but unassociated with obvious motor activity will establish that they represent a genuine cortical dis- 
charge rather than an artifact generated by sudden movement.

\section{EEG IN GENERALIZED EPILEPSIES}

The epileptic process in generalized epilepsies involves large areas of the brain at the outset of the seizure, and the EEG is characterized by bilaterally synchronous generalized paroxysms of spikes and spike wave discharges. Generalized epilepsies are subcategorized as primary (idiopathic) and secondary (symptomatic).

A patient with primary generalized epilepsy (PGE) has no identifiable etiology, normal brain imaging, and normal neurocognitive functioning. The epilepsy has a strong genetic basis and is highly responsive to antiepileptic medication. The patient may suffer from absence (petit mal), myoclonic, and tonic-clonic seizures, among other generalized seizures. Many different syndromes of PGE have been recognized depending upon the predominant seizure type and the age of onset. Classically, the presence of rhythmic, anteriordominant generalized bisynchronous $3 \mathrm{~Hz}$ spike wave discharges superimposed on a normal background are considered to be the EEG hallmark of PGE.

However, the most common EEG abnormality associated with PGE is the so-called "irregular" or "atypical" or "rapid spike" wave activity. This is characterized by generalized paroxysms of spikes or spike wave complexes occurring with an irregular frequency of about 3 to $5 \mathrm{~Hz}$. Although some spike wave complexes will approximate $3 \mathrm{~Hz}$, the overall impression is that the EEG abnormality is much less regular than the classic 3 $\mathrm{Hz}$ spike wave discharges (Fig. 21). Transient asymme- try of the bisynchronous spike wave activity and isolated "focal" spikes are common. Atypical generalized spike waves are not only seen in PGE but also in secondary generalized epilepsies such as progressive myoclonus epilepsies of different etiologies.

Besides the presence of brief (1 to 3 seconds) generalized spike wave discharges, there are no interictal epileptiform abnormalities that are specific for individual syndromes included under PGE (childhood absence epilepsy, juvenile absence epilepsy, juvenile myoclonic epilepsy, epilepsy with myoclonic absences, and generalized tonic-clonic seizures on awakening). There are a few EEG features that are more common with certain syndromes: (1) polyspike wave discharges are more common with myoclonic epilepsies; (2) paroxysms of occipital-dominant rhythmic delta activity in the EEG is a feature most commonly encountered with childhood absence epilepsy; (3) short paroxysms of spike wave discharges of higher frequency (4.0 to $4.5 \mathrm{~Hz}$ ) are more common with PGE manifesting primarily with generalized tonic-clonic seizures; and (4) PPR is most common with juvenile myoclonic epilepsy.

In patients with $\mathrm{PGE}$, a routine EEG may capture one or more absence seizures or epileptic myoclonic jerks. In children an absence seizure may be induced during the EEG study by hyperventilation with characteristic generalized $3 \mathrm{~Hz}$ spike wave discharge, which is sustained for more than 3 seconds in duration. Epileptic myoclonic jerks are associated in the EEG with highamplitude generalized polyspike wave discharges in association with myoclonic jerks. Not well recognized is the fact that the EEG in patients with PGE may record

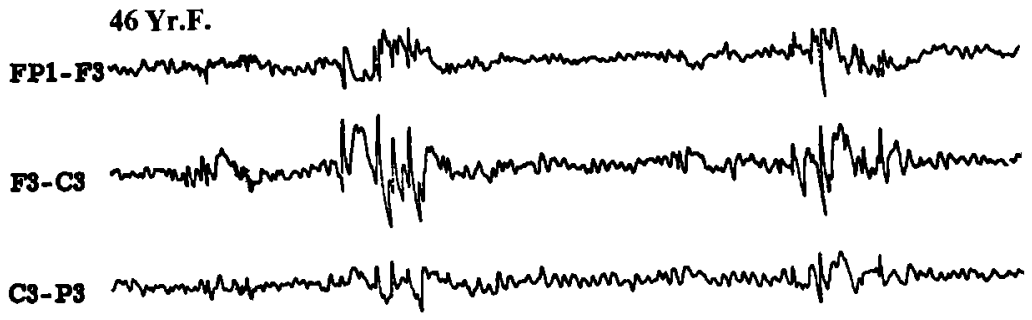

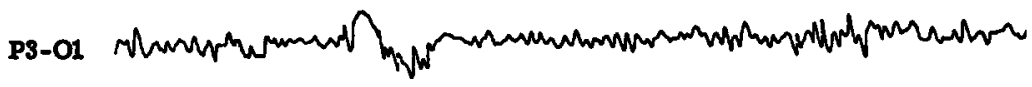

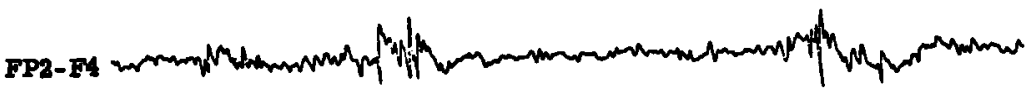

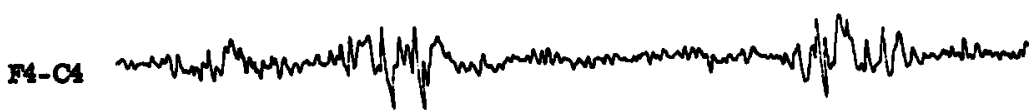

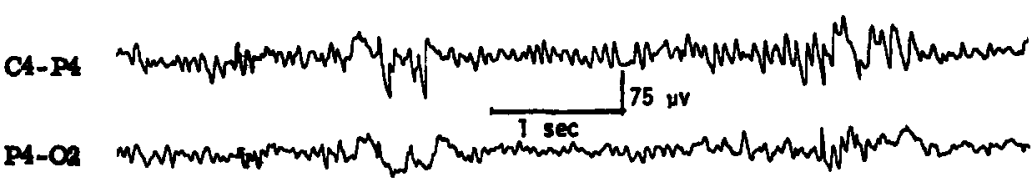

Figure 21 EEG of a 46-year-old patient with primary generalized epilepsy, showing atypical generalized bisynchronous spike wave activity. 
focal or lateralized spikes in addition to the overwhelming generalized bisynchronous spike wave activity. ${ }^{84,85}$ Similarly, spike or spike wave activity occurring bilaterally but restricted to the frontal areas (where the generalized paroxysms are usually maximum) is also common. Such discharges are often called "abortive" spike wave complexes. Roughly one quarter of patients with 3 $\mathrm{Hz}$ spike wave activity in their EEG may show such focal or lateralized discharges, ${ }^{84}$ which should generally be viewed as isolated fragments or limited expression of what is fundamentally a generalized epileptic abnormality. Such focal epileptiform discharges often shift from one electrode to the other and from one side to the other.

Secondary generalized epilepsy (SGE) is a more serious disorder, secondary to known diffuse cerebral hemispheric insult. Patients are children who have frequent seizures of generalized type, usually medically refractory. Many have significant developmental delay and neurocognitive deficits. In contrast to PGE, the background activity of the EEG in SGE syndrome is disorganized and there are variable degrees of slowing. In addition, there are several paroxysmal EEG patterns associated with SGE syndrome: (1) irregular bisynchronous spike wave activity described above, which can occur both with PGE or SGE; (2) slow spike wave (2.5 $\mathrm{Hz}$ or slower in frequency) discharges; (3) hypsarrhythmia; (4) independent multifocal spike discharges (IMSD); and (4) generalized paroxysmal fast activity (GPFA). These EEG patterns are largely nonspecific for etiology but are expressions of severe neocortical insult. Many of these EEG patterns are also age-dependent.

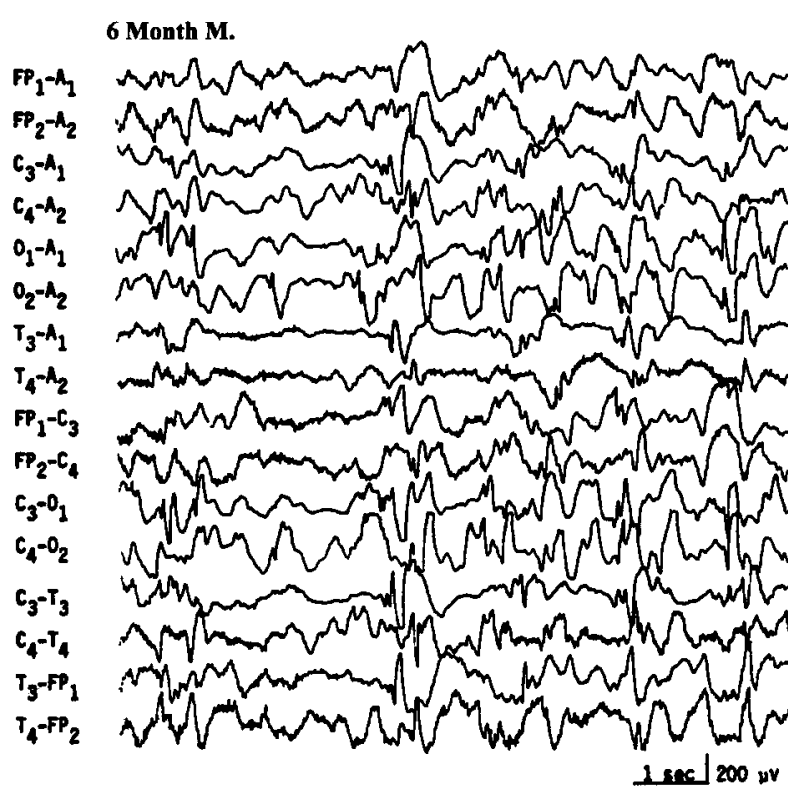

Figure 22 EEG of a 6-month-old infant with developmental delay and infantile spasms, showing typical hypsarrhythmic pattern.
West's syndrome (infantile epileptic encephalopathy) is characterized clinically by infantile spasms (jackknife seizures). The EEG usually shows a distinctive interictal pattern called hypsarrhythmia. It consists of very-high-amplitude, asynchronous slow activity superimposed on frequent multifocal spikes, polyspikes, or sharp waves or generalized spike wave complexes. The abundance of epileptiform activity, the entire absence of any organization ("chaotic" appearance), and absence of normal activities (e.g., alpha rhythm or sleep spindles) are constant features of a typical hypsarrhythmic pattern (Fig. 22). When some of the characteristic features are lacking or are less prominent, some would interpret the EEG as showing "modified hypsarrhythmia." Often the classical hypsarrhythmic pattern occurs only during NREM sleep, and the awake tracing showing diffuse slowing with minimal epileptiform activity. During an actual infantile spasm, there is an abrupt generalized attenuation of the background (i.e., an electrodecremental response) (Fig. 23). This may be preceded by a highvoltage, usually generalized biphasic slow wave complex. During the electrodecrement there may be lowamplitude beta activity with varying spatial distribution. These electrodecremental events occur often during sleep but without behavioral accompaniment.

Hypsarrhythmia, which is an EEG pattern, and infantile spasms do not have an absolutely constant relationship and are not interchangeable terms. Typical and modified hypsarrhythmia occurs in two thirds of the EEGs of infants with infantile spasms, whereas the remaining one third show generalized slow spike wave discharges (described below). ${ }^{86}$ Besides various pathologic conditions associated with a severe cortical insult, hypsarrhythmic pattern is often encountered in infants suffering from tuberous sclerosis or genetically determined metabolic conditions such as non-ketotic hyperglycenemia. ${ }^{87}$ Children with Aicardi's syndrome (agenesis of corpus callosum, mental retardation, infantile spasms, choreoretinal lesions) show a markedly asymmetric hypsarrhythmic pattern with virtually complete interhemispheric asynchrony of a suppression-burstlike background. ${ }^{88}$

The hypsarrhythmic pattern is a maturational pattern most commonly expressed between the ages of 4 and 12 months. As the infant grows older, beyond the age of 2 years, it is rare to encounter typical hypsarrhythmia, although infantile spasms may still continue. Hypsarrhythmia is replaced by different EEG patterns such as a diffusely slow tracing, slow spike wave discharges as seen with Lennox-Gastaut syndrome, IMSD, and, rarely, a normal tracing.

Adrenocorticotrophic hormone therapy often has a dramatic effect on infantile spasms as well as the hypsarrhythmic EEG pattern, which may virtually disappear in a matter of a few days to a few weeks after initiation of therapy. However, despite these clinical and 
5 Month.M.

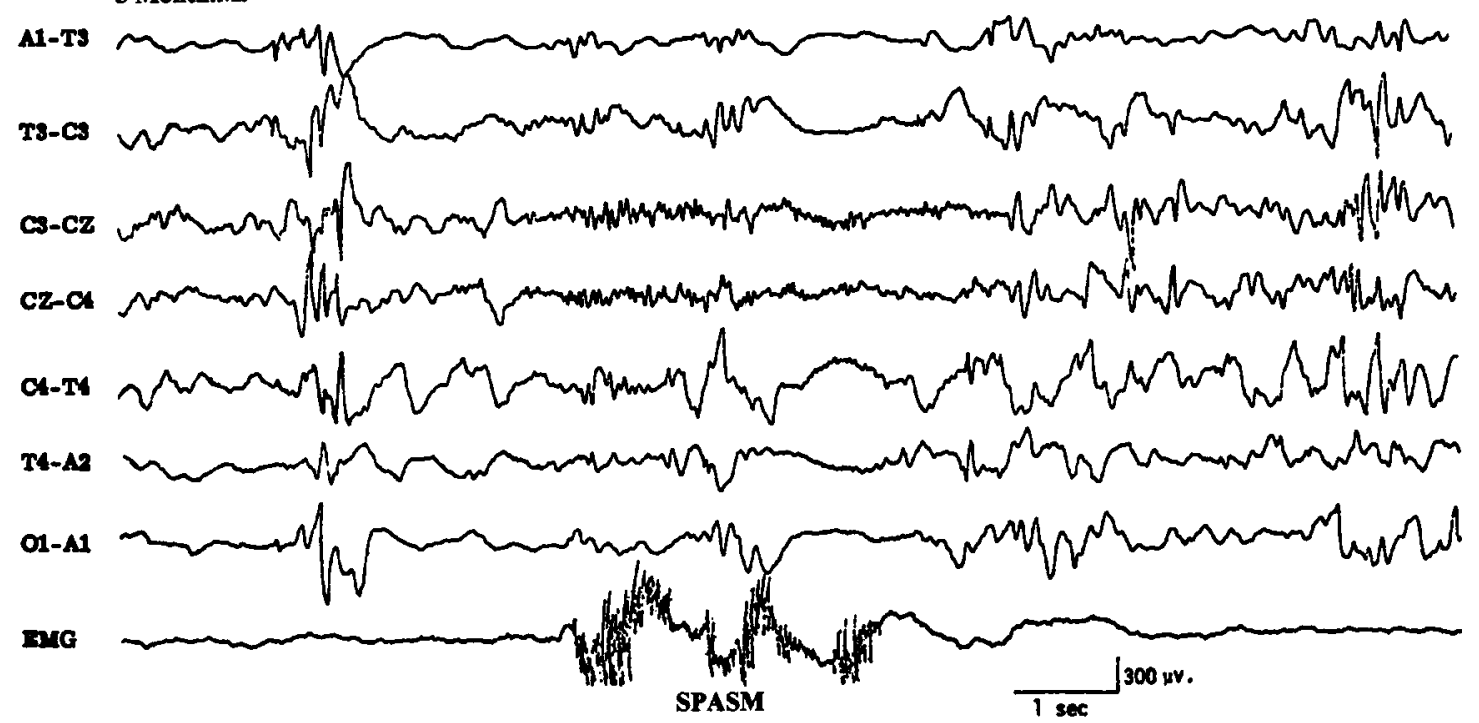

Figure 23 EEG of a 5-month-old infant, showing electrodecremental response during an infantile spasm monitored on the last channel.

EEG improvements, the long-term neurocognitive development remains subnormal.

Lennox-Gastaut syndrome (childhood epileptic encephalopathy) is another common form of SGE manifesting in early childhood with developmental delay, neurocognitive deficits, and frequent generalized seizures including tonic seizures. The EEG shows generalized, slow spike wave discharges (1.5 to $2.5 \mathrm{~Hz}$ ) su- perimposed on abnormally slow background activity (Fig. 24). ${ }^{89,90} \mathrm{It}$ is important to distinguish these EEG findings from those seen with primary generalized epilepsy where the background activity is normal for age and the generalized spike wave discharges are usually of faster frequency ( 3 to $5 \mathrm{~Hz}$ ). Although appearing widespread and bilaterally synchronous, the slow spike wave activity is usually higher in amplitude over the anterior

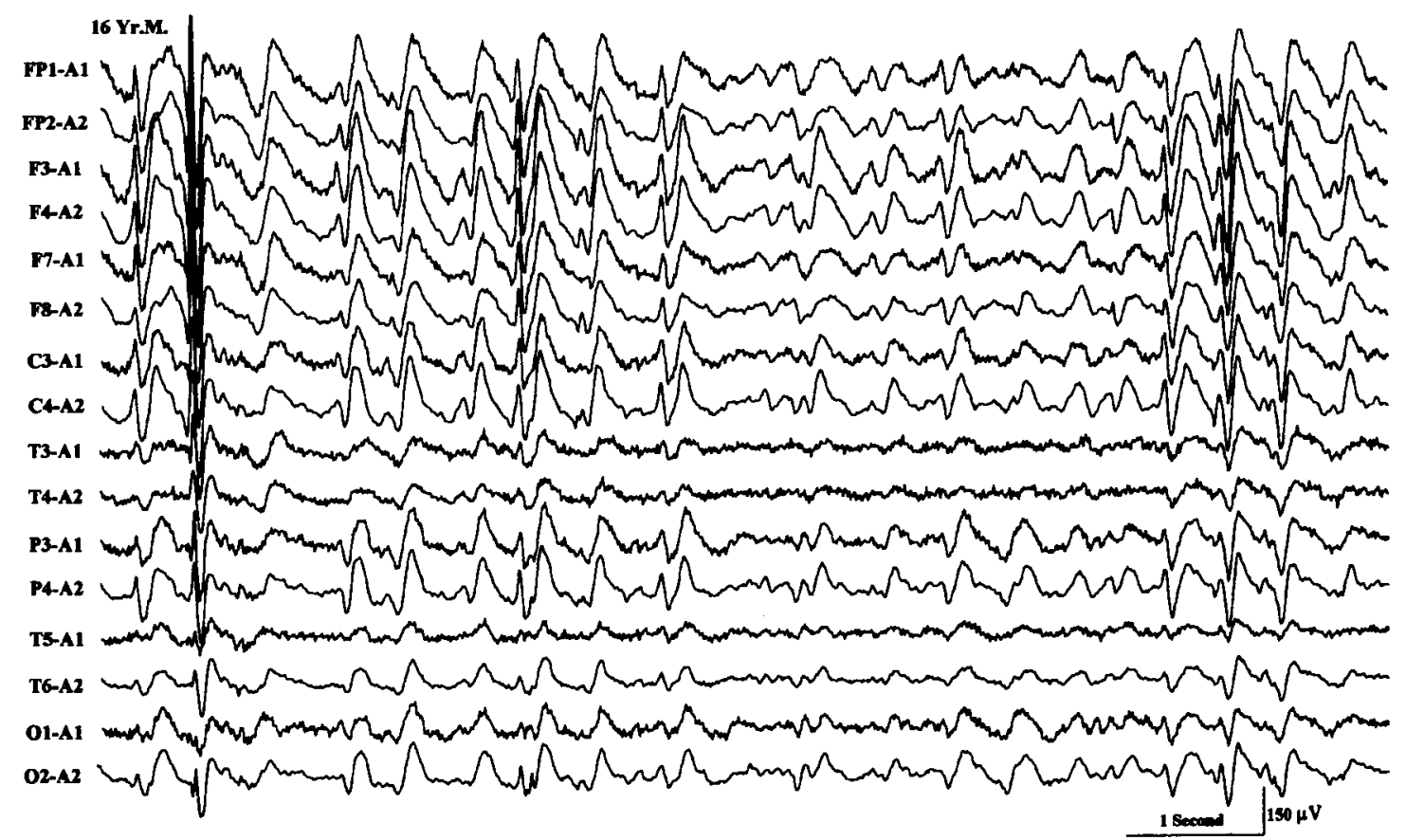

Figure 24 EEG of a 16-year-old child with mental retardation and tonic seizures, showing slow spike wave activity superimposed on a slow background. 
head regions (in $90 \%$ of patients); less commonly the amplitude is highest over the occipital areas. The duration of the paroxysms varies widely from isolated complexes to almost continuous slow spike wave activity, commonly without an identified behavioral or awareness change. Hence, the slow spike wave activity in Lennox-Gastaut syndrome is considered an interictal pattern, although it must be understood that subtle changes of behavior in retarded and uncooperative children are hard to recognize.

When one encounters prolonged episodes of slow spike wave activity lasting several seconds to minutes, the interpretative challenge is to decide if these electrographic events represent an ictal pattern (atypical absences or nonconvulsive status) or they simply represent more pronounced interictal pattern. A history of similar long episodes of slow spike wave activity in one or more previous EEGs would support an interictal finding. Also, giving a small dose of lorazepam intravenously will have no affect on an interictal pattern but will usually abort an ictal pattern, at least temporarily.

If a tonic seizure is recorded during the EEG of a patient with Lennox-Gastaut syndrome, the characteristic finding is an electrodecrement or "flattening" lasting several seconds. In addition, high-frequency rhythmic activity in the alpha-beta frequency range commonly occurs during the electrodecrement.

Another distinctive EEG pattern of a symptomatic generalized epilepsy syndrome is IMSD characterized by the presence of three or more independent and noncontiguous foci of spike or spike wave activity with at least one focus in one hemisphere (Fig. 25). ${ }^{91,92}$ As expected, the background activity is invariably disorganized and slow in frequency.

There is a close correlation between the three EEG patterns of hypsarrhythmia, slow spike wave, and IMSD associated with SGE. All of them are associated with diffuse or multifocal cerebral abnormalities and have similar clinical correlates of mental retardation, multiple and medically intractable seizure types, and a high incidence of neurologic deficits. ${ }^{91}$ Furthermore, serial studies over time may show a change of one pattern to the other in the same patient. Also, in the same EEG study, more than one of these patterns may coexist (e.g., IMSD during wakefulness and slow spike wave activity during sleep). It is very well known that at least $20 \%$ of infants with hypsarrhythmia may show slow spike wave usually by the second to fourth year of life. Both of these patterns may further change to IMSD in early childhood. Thus, these three EEG patterns have a common physiopathologic basis and are probably dependent more on cerebral maturation than on a particular kind of cerebral pathologic process. ${ }^{91}$ Hypsarrhythmia is usually seen in the later half of the first year of life in response to a cerebral insult prenatally, perinatally, or in the immediate postnatal period. It rarely results from cerebral insults after the second year of life. The slow spike wave pattern associated with Lennox-Gastaut syndrome is commonly observed between the ages of 2 and 5 years. The IMSD pattern is seen commonly throughout the first decade of life.

$7 \mathrm{~m}$. old

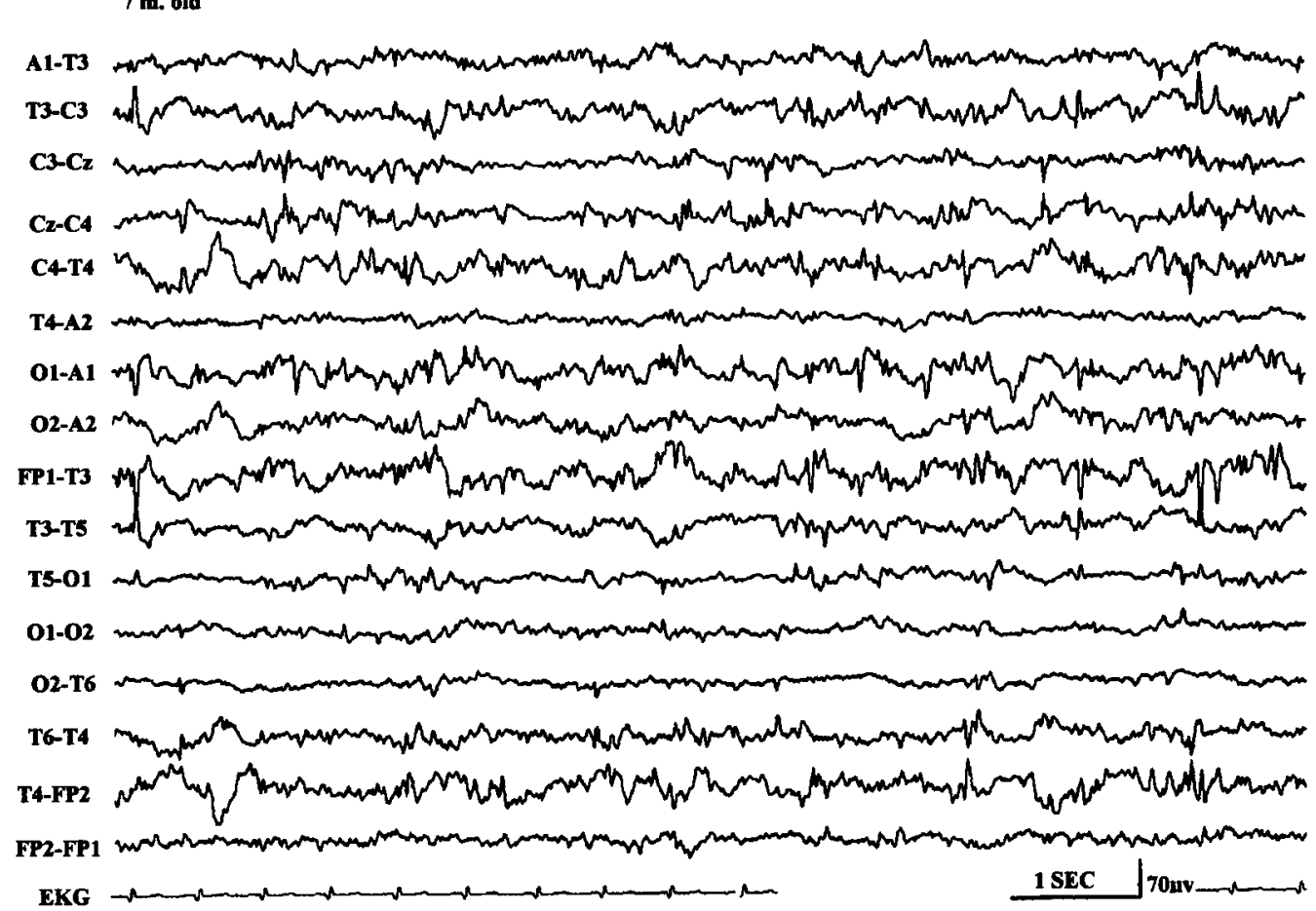

Figure 25 EEG of a 7-month-old child, showing independent multifocal spike discharges. 
$\mathbf{A}$

\section{1 yr. M.}

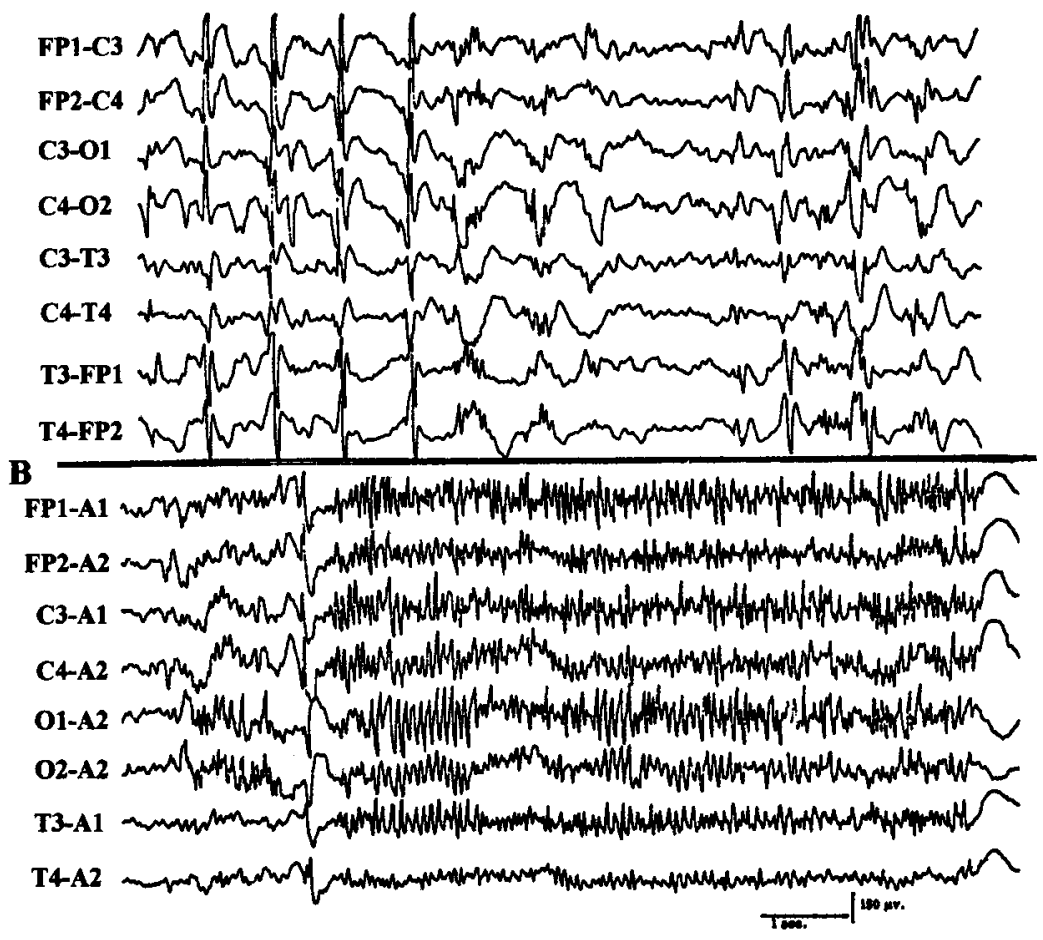

Figure 26 EEG of an 11-year-old patient with Lennox-Gastaut syndrome, showing generalized paroxysmal fast activity (B).
A unique EEG pattern of GPFA is seen predominantly during sleep consisting of high-frequency, 12 to $25 \mathrm{~Hz}$ repetitive spike discharges occurring synchronously over both hemispheres (Fig. 26). ${ }^{93} \mathrm{It}$ is associated most commonly with SGE (usually LennoxGastaut syndrome) but it may rarely occur also with PGE or in patients with focal seizures, particularly with a frontal lobe focus. This EEG pattern is usually not associated with an obvious clinical change, although subtle tonic seizures (opening of eyes and jaw, eye deviation upward) may be missed. In rare patients with PGE and $3 \mathrm{~Hz}$ generalized spike wave, the awake EEG may appear rather benign but the presence of GPFA during sleep is a warning that more severe encephalopathy may be present. In such patients, motor seizures are common and the disorder is likely to persist in adulthood. ${ }^{94}$

\section{EEG in Focal or Localization-Related Epilepsies}

Focal epilepsies are usually categorized into two subgroups: (1) asymptomatic (secondary) localizationrelated epilepsies due to acquired focal cortical processes; and (2) idiopathic (primary) localization-related epilepsies, which are largely age-dependent and genetically based disorders, the best-known being benign Rolandic epilepsy (BRE).

The hallmark of focal epilepsy in the interictal EEG is the presence of a focus of epileptiform activity (i.e., spikes, spike waves, or spike wave complexes). The interictal paroxysmal activity is characteristically ran- dom, occurring at inconstant intervals. Intuitively, the positive correlation of an abundance of epileptiform discharges with a more frequent occurrence of clinical seizures would be expected. This is, however, not true. Abundance and rate of repetition of epileptiform discharges correlate very poorly with the frequency of clinical seizures. It is not uncommon to see rare interictal spikes in a patient who has frequent complex partial seizures. The reverse is also true, as in BRE where the child may have only rare clinical seizures but very abundant epileptiform discharges, particularly during sleep. An important fact regarding interictal epileptiform discharges is that they become much more frequent in the EEG immediately after a clinical focal seizure. ${ }^{77}$

Another characteristic of the interictal EEG is the presence of a focal abnormality of the background activity. This may take the form of intermittent low-voltage slow waves and attenuation of faster rhythm with the same localization as that of the epileptiform discharges. In general, when the focal epileptiform activity occurs along with abnormal focal organization, the possibility of a structural lesion is more likely and the focal epilepsy is most likely to be symptomatic. On the other hand, occurrence of random focal spikes without any accompanying features of focal disorganization of the background activity is usual with idiopathic or primary localizationrelated epilepsies such as BRE or benign occipital epilepsy.

The majority $(85 \%)$ of patients with complex partial seizures have temporal lobe onset, whereas a 


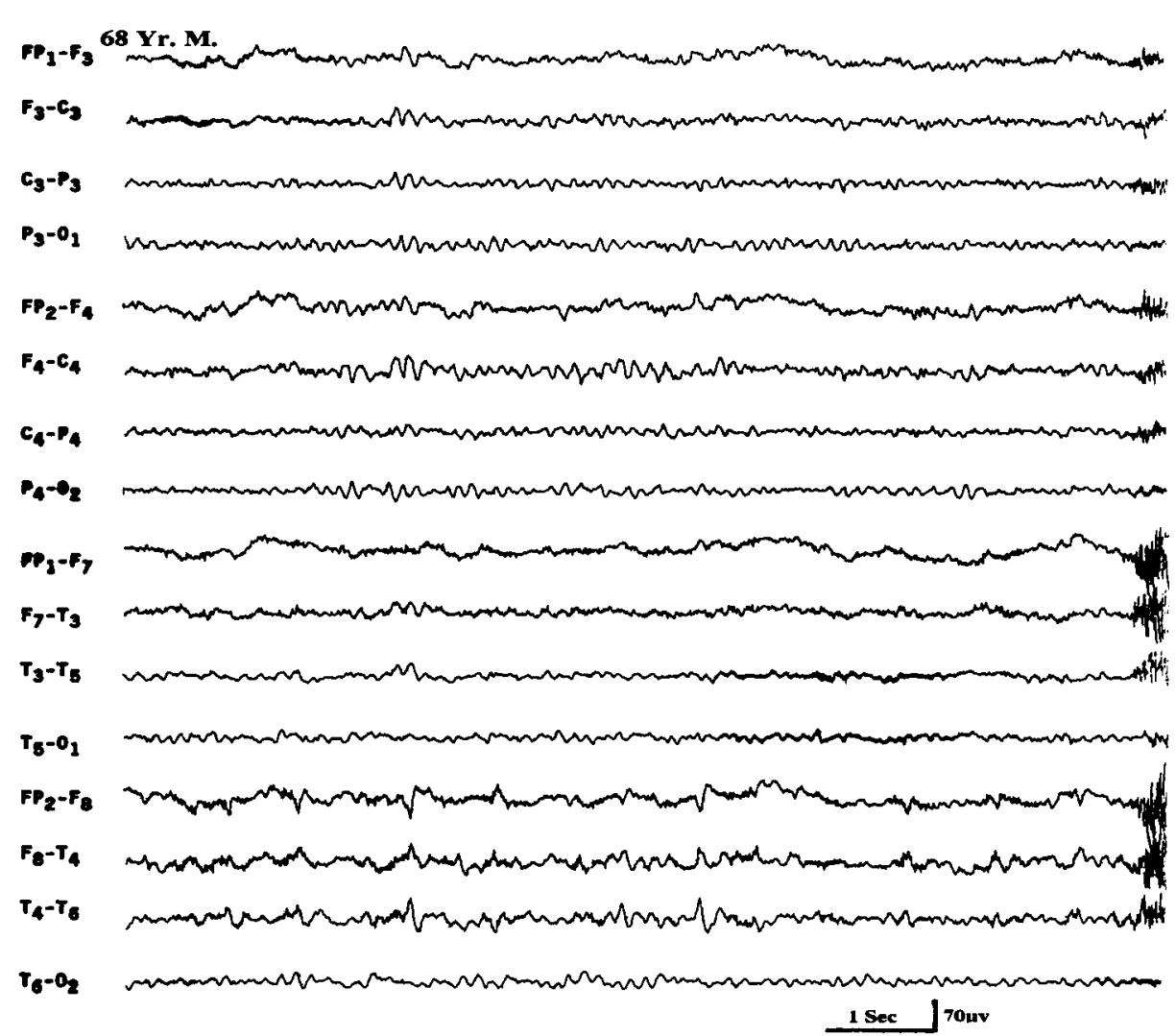

Figure 27 EEG of a 68-year-old patient with a long history of complex partial seizures, showing a focus of sharp waves and low-amplitude slow activity over the right anterior temporal region.

small proportion $(15 \%)$ have frontal lobe onset partial epilepsy. Temporal lobe epilepsy, which is the most common type of focal epilepsy in adults, is associated with interictal epileptiform discharges over one or both anterior temporal regions (Fig. 27). The peak of the potential field involves inferior frontal (F7 or F8), midtemporal (T3 or T4), and the ear lobe electrodes (A1 or A2). The F7 and F8 electrodes of the 10 to 20 electrode placement system more commonly record activity originating in the anterior temporal lobe rather than in the frontal areas. In suspected patients with temporal lobe epilepsy, recording from additional TI and T2 electrodes must be performed to optimally elicit epileptiform abnormalities of temporal lobe origin. In patients undergoing presurgical evaluation, anterior sphenoidal electrodes are commonly inserted, which markedly increase the yield of demonstrating temporal epileptiform abnormalities. In frontal lobe epilepsy, interictal epileptiform discharges are recorded over the frontal region but often the EEGs, even on several occasions, may remain normal. ${ }^{95,96}$ Special attention must be paid to the midline electrodes (FZ and CZ), which can demonstrate lowamplitude epileptiform discharges. Also, additional supraorbital and midfrontopolar (FPZ) electrodes may be helpful in demonstrating epileptiform abnormalities in frontal lobe epilepsy.
BRE constitutes the most common primary (idiopathic) localization-related partial epilepsy with onset between the ages of 4 and 10 years. Virtually all recover by the age of 15 or 16 years when the patients become seizure-free and their EEGs revert to normal. The background EEG activity in wakefulness and sleep is normal. There are epileptiform discharges over the central or centrotemporal region, which are markedly activated during sleep (Fig. 28). ${ }^{97}$ In some children, epileptiform discharges are restricted to sleep recording only.

A horizontal dipole field is a highly characteristic feature of the centrotemporal epileptiform discharges associated with BRE. ${ }^{98}$ The negative end of the dipole is located at the centrotemporal area, and the positive end toward the frontal regions bilaterally (see right half of Fig. 28). In 10 to $15 \%$ of patients with BRE, additional EEG abnormalities are seen. Independent spike foci may occur over the occipital and less commonly over the frontal regions. Interestingly, bisynchronous spike wave discharges also occur in approximately 10\% of patients with BRE during routine EEG recordings. ${ }^{99}$

Centrotemporal or Rolandic spikes are not specific for BRE. Children with acquired brain insults involving fronto-centro-temporal regions (who may also have neurologic deficits, e.g., cerebral palsy) may show epileptiform activity over one or both central areas. In contrast to BRE such "lesional" patients usually have 

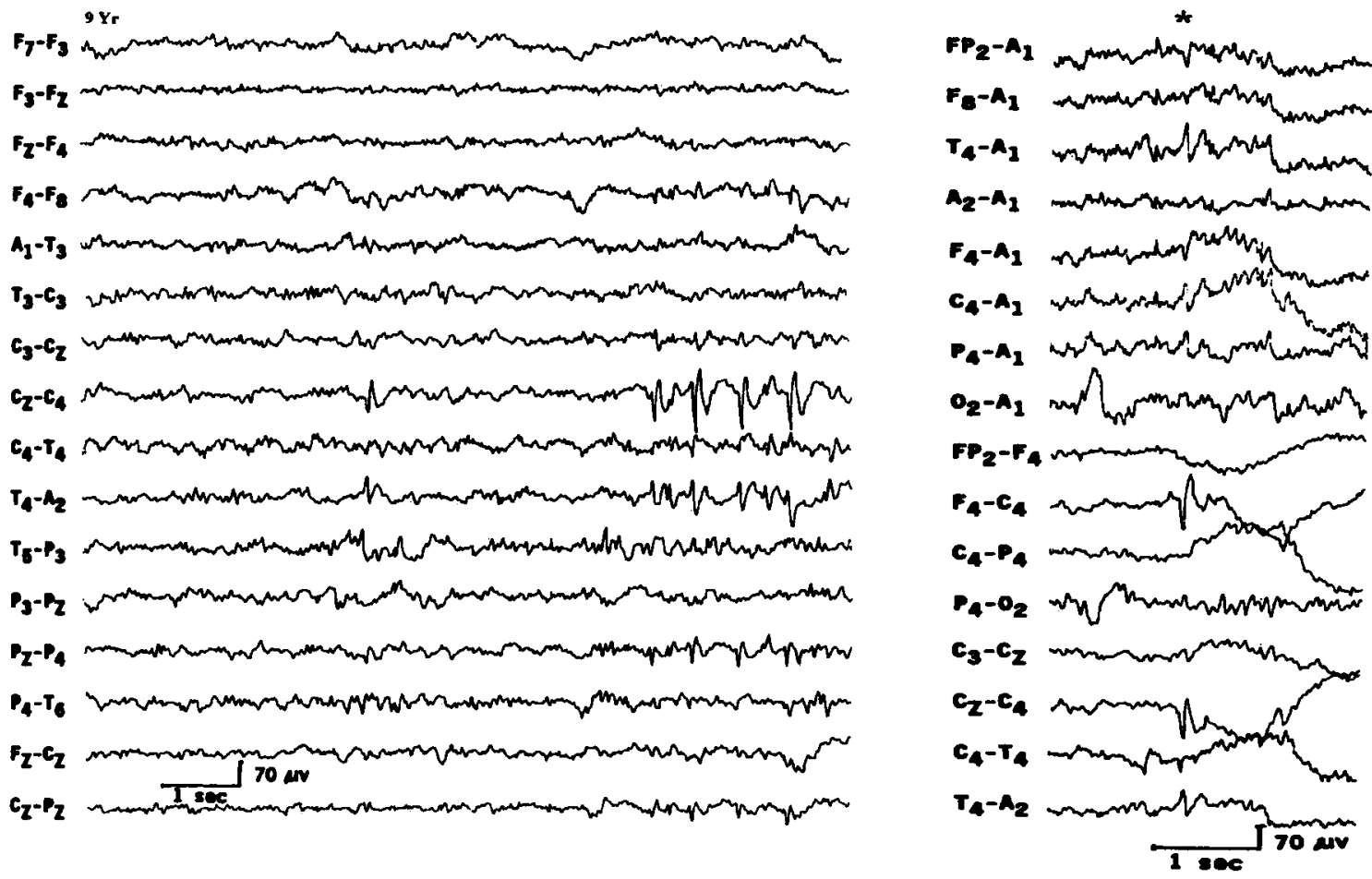

Figure 28 EEG of a 9-year-old child with benign Rolandic epilepsy, showing a focus of right centrotemporal spike discharges. The right half of the figure shows a spike discharge with horizontal dipole distribution.

abnormal background activity, either focally or diffusely, and have secondary (symptomatic) partial epilepsy.

Many studies ${ }^{100-102}$ have stressed that a small proportion of epileptics have midline spike foci; the epileptiform abnormalities are localized at the midline electrodes with some spread to parasagittal electrodes on both sides. They are best demonstrated in the coronal montage, which includes midline (FZ, CZ, and PZ) electrodes; the amplitude may be somewhat asymmetric in parasagittal electrodes. The midline spike foci have highest amplitude at the $\mathrm{CZ}$ electrode in most of the patients. Such foci are common in children, and at least half of the patients show these discharges only during sleep. Certainly, caution must be exercised in distinguishing midline $\mathrm{CZ}$ spikes from normal sleep potentials such as vertex sharp transients. Midline spike foci have a high correlation with clinical seizures of focal or generalized type, and a significant proportion show evidence of cognitive or neurologic deficits.

Rarely, a focal seizure is recorded in a routine EEG in a patient with focal epilepsy. As mentioned before, the hallmark of an ictal EEG pattern associated with a focal seizure is the sudden appearance of focal rhythmic activity that evolves during a short period with a progressive change in the amplitude and frequency with a topographic spread. Postictally, there may be a period of "flattening" followed by variable degrees of slow wave activity, which may be generalized initially but usually becomes lateralized or focal later in the pos- tictal period before the preictal EEG pattern becomes reestablished. A transient delta focus after the seizure is a very reliable sign of a localized lesion or at least a focal origin of the previous epileptic seizure. The postictal focal slowing may persist for a few seconds to a few hours or even a few days, depending upon such factors as the age of the patient, size of the lesion, duration, and number of seizures.

The ictal EEG pattern of complex partial seizures of temporal lobe onset usually starts with a focal rhythmic 4 to $7 \mathrm{~Hz}$ theta activity over one temporal region, which then spreads bilaterally, becoming higher in amplitude, slower in frequency, and often interspersed with sharp wave components.

Complex partial seizures of frontal lobe onset have a unique semiology compared with the complex partial seizures of temporal lobe onset. ${ }^{95,96}$ The former are brief (less than 1 minute), commonly nocturnal, often occurring in clusters with many per day, have complex motor automatisms with kicking and thrashing, sexual automatism, vocalization, and with very short or no postictal period of confusion after the seizure. The frantic and often bizarre motor thrashing and kicking behavior and rapid return of awareness at the end of the seizure frequently leads to an erroneous diagnosis of pseudoseizures. Diagnostic difficulty is further compounded by the fact that routine EEG, even on many occasions, may be nonrevealing in patients with frontal lobe seizures. ${ }^{95,96}$ Interictal abnormalities, when 
present, are often seen near the vertex bifrontally so that localization is not easy. Furthermore, the ictal EEG during a frontal lobe complex partial seizure often fails to provide diagnostic information because of marked contamination with ongoing movement artifacts during the seizure. Careful evaluation of the ictal EEG using coronal montage and high-frequency filtering may reveal rhythmic ictal patterns in frontal/central electrodes near the midline, spreading bilaterally to the parasagittal region. The frontal lobe complex partial seizures not only resemble pseudoseizures clinically but also lack diagnostic interictal and ictal EEG findings. Their brief duration, emergence out of sleep, and stereotypic semiology are highly characteristic features that help distinguish them from pseudoseizures.

\section{Certain EEG Patterns Morphologically Similar to Interictal/ictal Epileptiform Discharges But Unrelated to Epilepsy}

There are some EEG patterns that, by virtue of having spike or sharp wave components or being rhythmic, closely resemble diagnostically important epileptiform discharges. Although they share some of the same electrographic features, their presence in the EEG has no correlation with epileptic seizures. These patterns have been reviewed in detail. ${ }^{103}$

1. The 14 and $6 \mathrm{~Hz}$ positive spikes are brief paroxysms, usually less than 1 second, of positive spikes with a frequency of $6 \mathrm{~Hz}$ at some times and $14 \mathrm{~Hz}$ at others. They are distributed over the posterior hemispheric regions, occurring usually unilaterally. If enough of them are recorded in a tracing, they are expressed on both sides of the head. They are most commonly seen in children and adolescents during drowsiness and light sleep. This pattern has been the subject of many reviews and long-drawn controversy but it is now held to be a normal sleep activity or a normal variant.

2. Small sharp spikes or benign epileptiform transients of sleep are spike potentials that are recorded in adults during drowsiness and light sleep. They are sporadic and shift from one side to the other, and have widespread distribution over the scalp despite low amplitude. The spikes are usually low amplitude (less than $50 \mu \mathrm{V}$ ), very short duration (less than 50 milliseconds), and mono- or diphasic potentials with no or minimal slow wave component following the spikes (Fig. 29). They are never associated with focal slowing or other abnormalities of the background activities. Their special distribution on the scalp may suggest a horizontally oriented dipole generator extending across the sagittal midline with opposite polarity on the two sides of the head. They are especially frequent in the EEG performed after a period of sleep deprivation and are considered to have no correlation with epileptic seizure disorder. ${ }^{104}$

3. The $6 \mathrm{~Hz}$ spike wave or phantom spike wave consists of bilaterally synchronous, short (less than 1 second) paroxysms of 5 to $7 \mathrm{~Hz}$ spike wave activity. ${ }^{105} \mathrm{Al}-$ though widespread in distribution, they are usually

72 Yr. F.

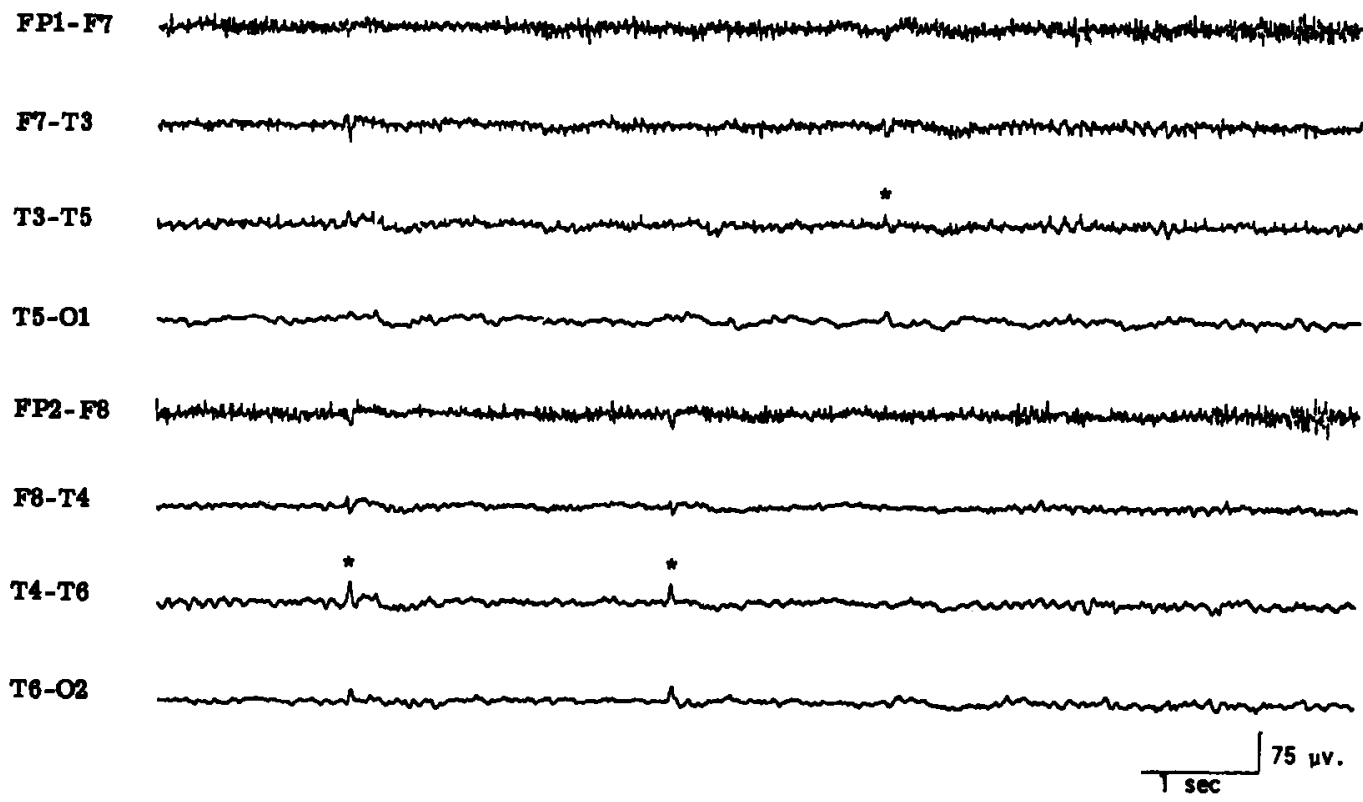

Figure 29 EEG of a 72-year-old patient, showing benign epileptiform transients of sleep (small sharp spikes). 


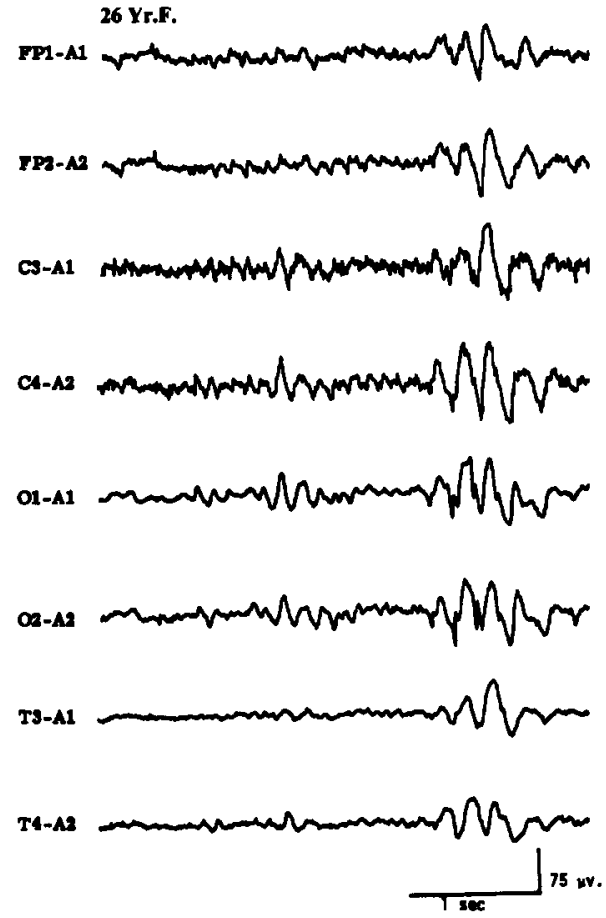

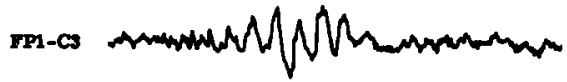

FPa-ce

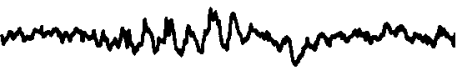

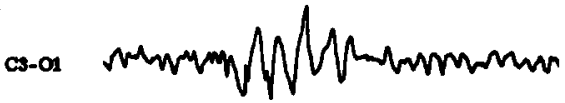

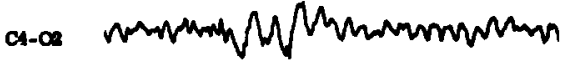

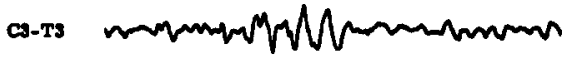

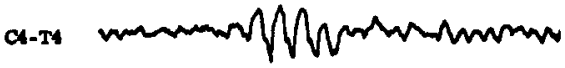

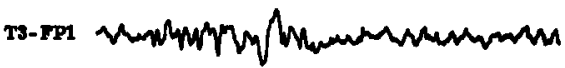

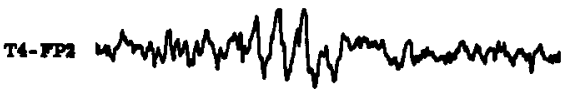

Figure 30 EEG of a 26-year-old patient, showing $6 \mathrm{~Hz}$ spike wave paroxysms (phantom spike wave).

best developed over the posterior hemispheric region (Fig. 30). The spike component is usually low in amplitude, whereas the slow wave following it is more prominent; hence, the term "phantom spike wave." It occurs in young adults and is best expressed during drowsiness. There is still some controversy regarding their clinical significance, especially the ones that are anteriorly dominant. It has been suggested ${ }^{106}$ that the $6 \mathrm{~Hz}$ spike wave activity that occurs with high amplitude and predominance over the anterior hemi- sphere and is recorded during wakefulness may have a high correlation with seizures, whereas predominantly occipital, low-amplitude, spike wave discharges occurring in drowsiness have no correlation with epileptic seizures.

4. "Psychomotor variant" or rhythmic midtemporal discharges is a rare EEG finding observed in less than $1 \%$ of the patient population coming to an EEG lab. It is characterized by long (several seconds to a minute) paroxysms of 5 to $7 \mathrm{~Hz}$ rhythmic activity

11 Yr.F.

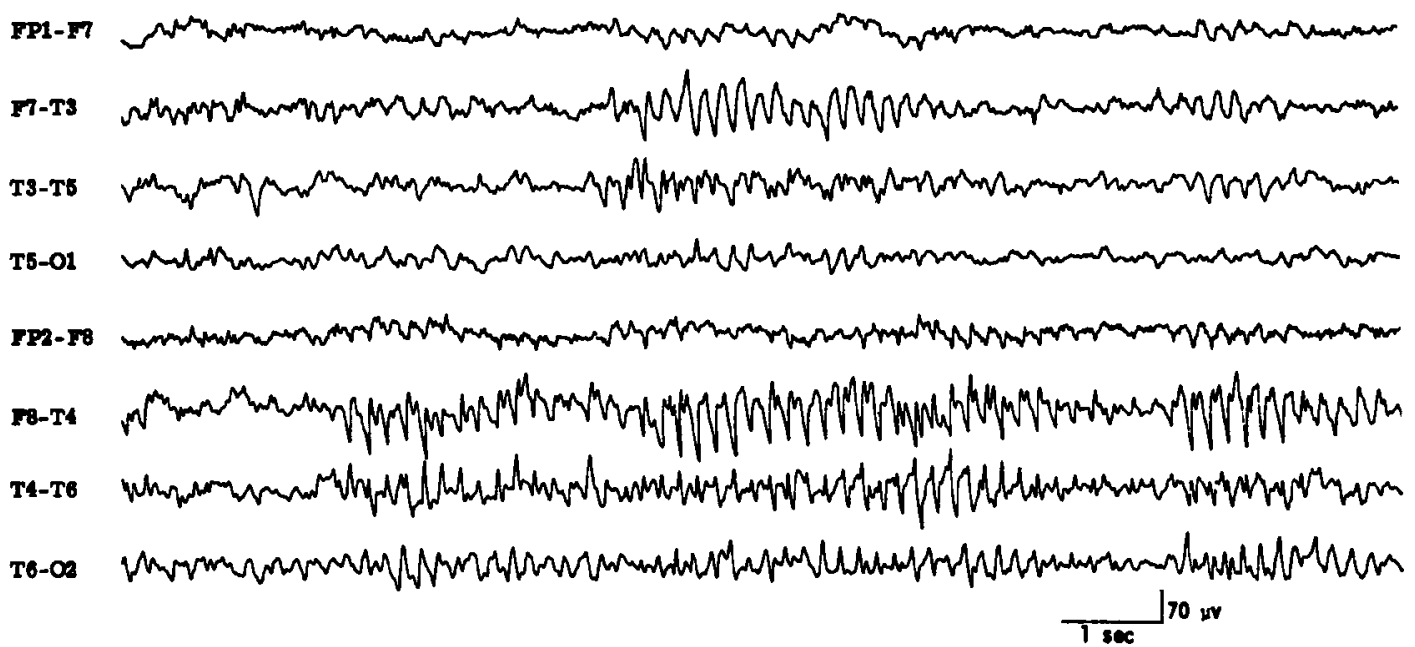

Figure 31 EEG of an 11-year-old patient, showing rhythmic midtemporal discharges during drowsiness. 
with an admixture of sharp components occurring over the midtemporal (T3/T4) regions (Fig. 31).107 The paroxysms are unilateral or bilateral. Bilateral paroxysms may appear independently on the two sides or simultaneously with variable asymmetry. The pattern is invariant and without evolution from beginning to end. This EEG finding occurs during drowsiness or light sleep and has no correlation with epileptic seizure disorder.

5. The wicket temporal pattern is characterized by negative sharp transients that occur in runs and have a wicket shape. The repetition rate varies between 6 and $11 \mathrm{~Hz}$ in different and even in the same bursts. They are best seen in T3/T4 or F7/F8 electrodes with variable spread to A1/A2. They are usually bitemporal, occurring independently on the two sides. The pattern occurs exclusively in adults and is seen in both wakefulness and sleep. ${ }^{108}$

6. Subclinical rhythmic EEG discharges of adults is a rare EEG pattern ${ }^{109,110}$ that closely resembles an ictal pattern, but there is no evidence that it represents an epileptic seizure. It occurs mainly in elderly subjects and may extend beyond 1 minute but without any clinical change. It is predominant over the posterior head regions and usually bilateral but may be asymmetric. In the most characteristic form the pattern begins with a series of rhythmic sharp-contoured waves that gradually merge into a sustained theta frequency. It may subside abruptly or gradually. ${ }^{103}$

7. There are many normal activities, particularly during sleep in children that, being sharp in configuration, are often mistaken for epileptiform discharges. These include vertex sharp transients and positive occipital sharp transients. In children, the vertex sharp transients may be quite high in amplitude and spiky in configuration, very closely resembling epileptiform discharges. One has to be very careful in interpreting sharp waves or spikes in children of Rolandic location expressed only during sleep. Normal mu rhythm in central leads and breach rhythm recorded in patients with iatrogenic or acquired skull defect may also consist of sharp components that may be mistaken for epileptiform discharges. Lambda waves, which are sharply contoured activity in the occipital region seen when a person has eyes open and scans the surrounding environment, need to be differentiated from posterior hemispheric epileptiform discharges.

\section{EEG IN NEONATES}

In recent years there has been much interest in using EEG to evaluate full-term or premature neonates ${ }^{111,112}$ due to the serious limitations in performing an adequate neurologic examination. The neonate may be confined to an isolette, may be intubated, or may be paralyzed for ventilatory control. Under such circumstances, EEG is a very important tool to assess an encephalopathic process or occurrence of epileptic seizures. In addition, the background abnormalities have been classified in neonates and used to predict neurologic outcome.

The EEG of a neonate shows distinctive patterns related to the conceptional age (CA) and the behavioral state (awake, active sleep, quiet sleep). Space does not permit a description of EEG patterns associated with different conceptional ages, but this is summarized in Table 2. It is important to emphasize that the EEG maturation runs parallel in utero and in incubator; only minimal or no differences have been found between babies of the same CA born after different periods in utero.

In a full-term neonate four EEG patterns are observed related to the wakefulness/sleep cycle: (1) lowvoltage irregular (LVI) is characterized by the presence of continuous low-amplitude $(<50 \mu \mathrm{V})$, mainly widespread theta activity; (2) mixed pattern is characterized by continuous moderate amplitude (usually $<100 \mu \mathrm{V}$ ) theta and delta activities; (3) high-voltage slow (HVS) consists of continuous high-amplitude semirhythmic, mostly delta activity ( 0.5 to $3.0 \mathrm{cps}$ ) in all regions with an amplitude of 50 to $150 \mu \mathrm{V}$; and (4) tracé alternant (TA) pattern is characterized by the occurrence of 3 to 5 second bursts of high amplitude $(50$ to $100 \mu \mathrm{V})$ slow activity ( 0.5 to $3.0 \mathrm{~Hz}$ ), which occur at intervals of 3 to 10 seconds when the background is relatively low amplitude $(10$ to $25 \mu \mathrm{V})$ consisting of theta waves. In other words, there is an alteration of bursts of large-amplitude slow activity separated by quiescent or "flat" periods of low-voltage activity.

These four EEG patterns are recorded in different states. The LVI EEG is usually recorded in wakefulness and in active sleep. The mixed pattern can also be recorded in active sleep and relaxed wakefulness. The TA and HVS patterns are characteristic of quiet sleep. A unique characteristic of neonate sleep is that as the neonate falls asleep, he usually enters a period of active (REM) sleep. This differs from older infants, children, and adults who never begin their sleep with a period of active (REM) sleep. It is only by 10 to 12 weeks postterm that sleep onset changes from active to quiet (NREM) sleep.

A couple of perils and pitfalls need emphasized in interpreting the neonatal EEG. The TA pattern of quiet sleep in a normal full-term neonate and tracé discontinua pattern in a normal premature neonate during quiet sleep have a superficial resemblance to the suppression-burst pattern that carries a poor prognosis. Differentiation between normal and abnormal discontinuous patterns becomes an important challenge in interpreting the neonatal EEG. The suppression-burst pattern is invariant and nonreactive to stimulation. It usually signifies a severe encephalopathy (usually is- 
Table 2 EEG Maturation in Preterm Neonates

\begin{tabular}{|c|c|c|c|c|}
\hline EEG Features & 24-27 Weeks CA & 28-31 Weeks CA & 32-35 Weeks CA & 36-38 Weeks CA \\
\hline Continuity & $\begin{array}{l}\text { Discontinuous, long } \\
\text { flat periods or tracé } \\
\text { discontinua }\end{array}$ & $\begin{array}{l}\text { Discontinuous or } \\
\text { tracé discontinua }\end{array}$ & $\begin{array}{l}\text { Discontinuous } 50 \% \text { of } \\
\text { time, more or less } \\
\text { continuous } 50 \%\end{array}$ & $\begin{array}{l}\text { Continuous during } \\
\text { awake and AS; TA } \\
\text { during OS }\end{array}$ \\
\hline $\begin{array}{l}\text { Differentiation of } \\
\text { awake and sleep }\end{array}$ & No differentiation & No differentiation & $\begin{array}{l}\text { Wake/sleep diff. seen; } \\
\text { also AS/QS diff. } \\
\text { later in this period }\end{array}$ & Good \\
\hline Temporal theta & Present & Prominent & Decreasing & Absent \\
\hline Occipital theta & Prominent & Decreasing & Decreasing & Absent \\
\hline Delta brushes & None or minimal & $\begin{array}{c}\text { Prominent, } \\
\text { AS > OS }\end{array}$ & $\begin{array}{c}\text { Present, mainly in } \mathrm{QS} \text {, } \\
\text { less or none in AS }\end{array}$ & Rare in $\mathrm{OS}$ \\
\hline Tracé alternant (TA) & None & None & $\begin{array}{c}\text { Prominent during QS } \\
\text { (tracé discontinua) }\end{array}$ & Present in OS \\
\hline $\begin{array}{l}\text { Interhemispheric } \\
\text { synchrony }\end{array}$ & $\begin{array}{c}\text { Short bursts of } \\
\text { synchronous } \\
\text { EEG activity }\end{array}$ & $\begin{array}{l}\text { Prominent } \\
\text { asynchrony }\end{array}$ & $\begin{array}{l}\text { Progressively } \\
\text { more synchrony }\end{array}$ & $\begin{array}{r}\text { Minor asynchrony } \\
\text { may still persist }\end{array}$ \\
\hline $\begin{array}{l}\text { Sharp wave } \\
\text { transients }\end{array}$ & $\begin{array}{c}\text { Some (temporal) } \\
\text { sharp activity } \\
\text { during bursts }\end{array}$ & $\begin{array}{c}\text { Some scattered } \\
\text { sharp activity }\end{array}$ & $\begin{array}{l}\text { Often prominent } \\
\text { multifocal sharp } \\
\text { waves }\end{array}$ & $\begin{array}{l}\text { Sharp waves less } \\
\text { prominent, seen } \\
\text { over frontal/ } \\
\text { temporal during OS }\end{array}$ \\
\hline
\end{tabular}

AS, active sleep; $\mathrm{QS}$, quiet sleep; CA, conceptional age; diff., difference.

chemic/hypoxic), although it may occur transiently due to recent intravenous sedative/hypnotic medication. On the other hand, the TA and tracé discontinua pattern associated with quiet sleep in full-term and premature neonates, respectively, are state dependent and react to stimulation. In addition, long recordings would demonstrate activities characteristic of wakefulness and active sleep in normal full-term and premature (over 32 weeks $\mathrm{CA})$ neonates. The TA pattern gradually disappears over 6 weeks postterm when the HVS pattern becomes the sole EEG accompaniment of quiet sleep. ${ }^{113}$

Another striking feature of the neonatal EEG is the frequent occurrence of multifocal sharp transients during indeterminate and quiet sleep. These start appearing at 35 weeks $\mathrm{CA}$ and constitute a normal finding in full-term neonates. The clinical significance of these sharp transients remains controversial. It is difficult to clearly separate abnormal (pathologic) sharp waves from normal sharp transients of the neonate. In general, the normal sharp transients are infrequent in occurrence, usually blunt in morphology (rather than assuming spiky configuration), arise from any scalp location but are common over frontal and temporal regions, are truly random in occurrence, without persistent focality, and largely confined to the burst phase of the TA pattern. However, no universal criteria have been established to separate normal sharp transients of the newborn from the abnormal sharp wave activity. Unless the sharp wave discharges are repetitive, periodic, or localized over one region, an epileptogenic significance must not be assigned to them. When the multifocal sharp transients are very frequent and occur even during active sleep and wakefulness, the EEG is considered to be abnormal but suggestive merely of a nonspecific encephalopathic process.

A unique EEG pattern of pathologic significance is the presence of positive Rolandic sharp waves (PRS). ${ }^{114}$ PRS may be confined to one hemisphere; if they are bilateral they may be consistently more abundant over one hemisphere. They are usually maximum at $\mathrm{CZ}$ but may be lateralized to $\mathrm{C} 3$ or $\mathrm{C} 4$ electrodes (Fig. 32). Their positive polarity is a distinctive feature in addition to their localization. Although initially described as the EEG correlates of the intraventricular hemorrhage, PRS waves may be seen in other conditions, including periventricular leukomalacia, parenchymal hemorrhage, hydrocephalus, hypoxic/ischemic insult, and other conditions. ${ }^{112,115-117}$ Presently, the view is that PRS waves represent a marker of different white matter lesions rather than being specific for periventricular/intraventricular hemorrhage. Positive polarity sharp waves at other locations (especially in the temporal region) have no distinctive significance and may just be a part of multifocal sharp transients in the neonates.

Abnormalities of the background activity in fullterm neonates are usually classified as either severe or mild ${ }^{118-120}$ and are summarized in Table 3 . The severe EEG abnormalities indicate severe impairment of brain function and carry a poor prognosis for survival and/or neurologic development. Severely abnormal EEG patterns consist of: (1) isoelectric EEG showing activity 


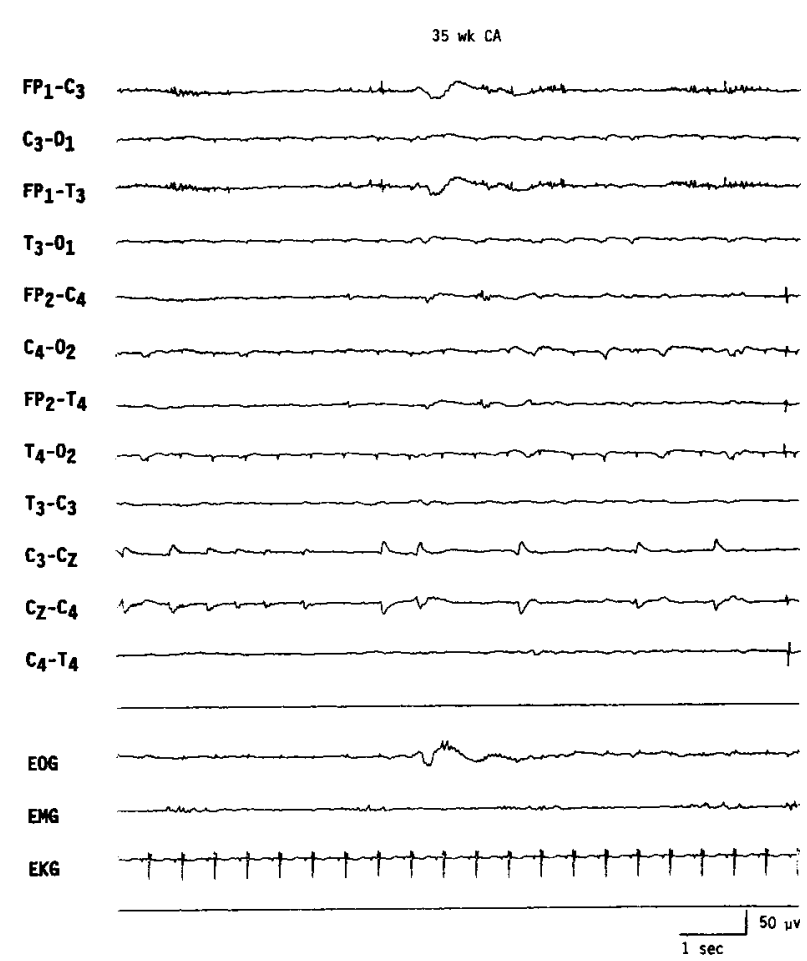

Figure 32 EEG of a neonate of 35 weeks conceptional age, showing low-amplitude positive Rolandic sharp wave discharges at the vertex (CZ) electrode. Patient had grade IV intraventricular hemorrhage and was unresponsive.

consistently below $5 \mu \mathrm{V}$; (2) persistent low-voltage tracing, EEG showing activity between 5 and $15 \mu \mathrm{V}$ with very little variability or sleep/wake differentiation; (3) paroxysmal tracing or suppression-burst pattern (discontinuous, invariant, and nonreactive pattern characterized by 1 to 10 second paroxysms of polymorphic activities such as sharp waves, spikes, and theta-delta

\section{Table 3 Classification of EEG Abnormalities}

\section{in Neonates}

1. Grossly abnormal background activities
a. Inactive (isoelectric) EEG
b. Low-voltage EEG
c. Tracé paroxystique (suppression-burst)
d. Invariant delta activity
e. Gross interhemispheric asynchrony/asymmetry

2. Mild abnormalities of background activities
a. Immature for CA
b. Excessive asynchrony/asymmetry
c. Lack of sleep states
d. Excessive discontinuity
e. Focal abnormalities
f. Miscellaneous, e.g., excessive frontal slowing, excessive frontal or multifocal sharp for $\mathrm{CA}$, excessive rhythmic activities, etc.

3. Positive Rolandic sharp waves

4. Ictal EEG patterns associated with neonatal seizures activities interspersed with long quiescent periods as long as > 20 seconds); (4) invariant high-amplitude delta activity (persistent and nonchanging high-amplitude 0.5 to $3.0 \mathrm{~Hz}$ generalized activity); and (5) the presence of gross asynchrony and asymmetry of the EEG activity over the two sides of the head. Studies have established that the presence of these EEG abnormalities in a full-term neonate, particularly if the abnormalities have been persistent in serial EEGs, carry a very poor prognosis for survival or future neurologic development; over $90 \%$ of neonates with such severe abnormalities have an unfavorable outcome. ${ }^{118,119}$

Mild abnormalities of the background activities include more than the usual asynchrony and/or asymmetry; EEG being immature for the conceptional age; lack of recognizable sleep states; excessive discontinuity ("flat" periods longer than 30 seconds); abnormal monorhythmic activities; and excessive multifocal sharp transients. The presence of more than one mild abnormality may suggest an underlying encephalopathic process of varying severity, particularly if the EEG shows persistent abnormality on serial studies. Several of the above mild abnormalities do occur in neonates who are heavily sedated; hence, iatrogenic causes need to be excluded before ascribing them to permanent neurologic insult.

\section{Neonatal Seizures}

One of the major reasons an EEG is performed is if a neonate is suspected of having epileptic seizures. In neonates, epileptic seizures are often characterized clinically by subtle motor behavior such as elevation of a limb, eye deviation, eyelid flutter, tonic posturing, bicycling movements of the legs, apnea, and so on. The EEG is indispensable in establishing the epileptic nature of the motor activity by demonstrating an associated ictal pattern. There are many unique features of neonatal seizures that are different from the seizures encountered in older children and adults. The International Classification of Epileptic Seizures is obviously inappropriate for neonates. The immature brain at this age is unable to initiate and sustain generalized epileptic discharges as in older children; hence, typical tonicclonic seizures do not occur. Many of the neonatal seizures are subtle seizures as described above. At least some of these do not show a close relationship to an EEG change. The significance of such stereotypic motor events with no concomitant EEG changes becomes a controversial issue regarding diagnosis and management. Whether these events represent "epileptic" dysfunction (not "picked up" by scalp electrodes) or whether these stereotypic behaviors signify episodes of brain stem release phenomena has yet to be resolved.

The opposite situation is also common. An electrographic ictal pattern may occur without an obvious 
clinical change. Such "subclinical” seizures are common with a pharmacologic neuromuscular blockade, stupor and coma following severe hypoxic/ischemic encephalopathy, multifocal status epilepticus, and following apparently successful treatment of status epilepticus using antiepileptic drugs.

The EEG ictal pattern is highly variable but consists of rhythmic activity of some sort, which is well localized to a relatively small area of the brain. With rare exception, the ictal pattern in neonates is focal, unifocal, or multifocal. When multifocal, the ictal pattern may occur over two different regions at the same time but the discharges have different waveforms and different repetition rates. Multifocal seizures simultaneously occurring on the two sides is a unique feature of neonatal epileptogenesis.

Interictal epileptiform abnormalities are rarely present to aid in the diagnosis of neonatal seizures. Multifocal sharp transients over the frontal and temporal regions are common even in healthy neonates and, as mentioned above, do not correlate with present or future occurrence of epileptic seizures. It appears that in neonates the epileptic process exhibits "all or none" features: either a seizure manifests overtly with appropriate electrographic features or has no interictal epileptiform markers.

Ictal EEG patterns associated with neonatal seizures are of four basic types ${ }^{111}$ :

1. Focal spikes or spike wave discharges superimposed on a more or less normal background is an ictal pattern most commonly located over the Rolandic re- gion $(\mathrm{C} 3$ or $\mathrm{C} 4)$, and the frequency of the ictal discharge is usually over two per second (Fig. 33). In neonates, focal EEG discharges and clinical seizures do not necessarily imply focal brain lesions. Common etiologies include metabolic disturbances, such as hypocalcemia or hypoglycemia, and subarachnoid hemorrhage. Such an ictal pattern, when associated with normal a EEG background, is prognostically favorable.

2. Another focal ictal pattern consists of slow-frequency sharp waves or complex waveforms repeating approximately one per second, never recruiting at a faster rate (Fig. 34). This pattern is similar to PLEDs. The background activity is almost always low in amplitude. This pattern, called "depressed" brain discharges, ${ }^{121}$ is associated with a severe cerebral insult (e.g., hypoxic/ischemic encephalopathy, encephalitis, cerebrovascular accident, etc.). The accompanying clinical seizures are usually subtle or the EEG discharges are entirely subclinical.

3. Focal monorhythmic pattern in the beta, theta, and delta frequency is a unique ictal pattern in neonates. It may start with low-amplitude focal 8 to $14 \mathrm{~Hz}$ activity that slows down to 4 to $7 \mathrm{~Hz}$ and then to 0.5 to 3.0 $\mathrm{Hz}$ rhythmic pattern. All types of combinations of the frequency band are possible but some ictal discharges may remain essentially monorhythmic ("alpha band" seizures) during a given seizure (Fig. 35). The background is always abnormal and usually low in amplitude. This type of pattern has been referred to as pseudo-beta-alpha-theta-delta ictal pat-

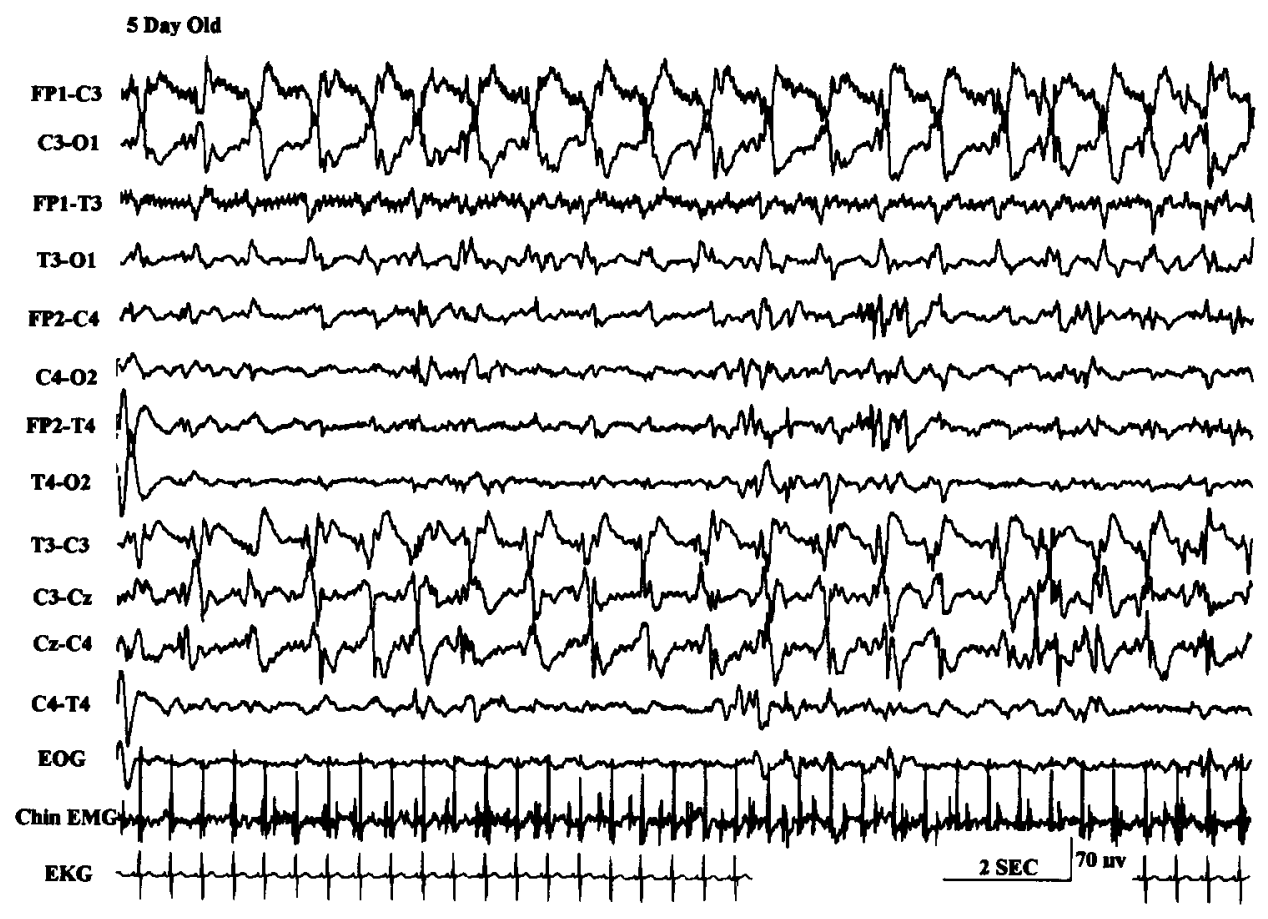

Figure 33 EEG of a 5-day-old neonate, showing focal ictal pattern characterized by rhythmic sharp waves in the left Rolandic region. 


\section{D. Old}

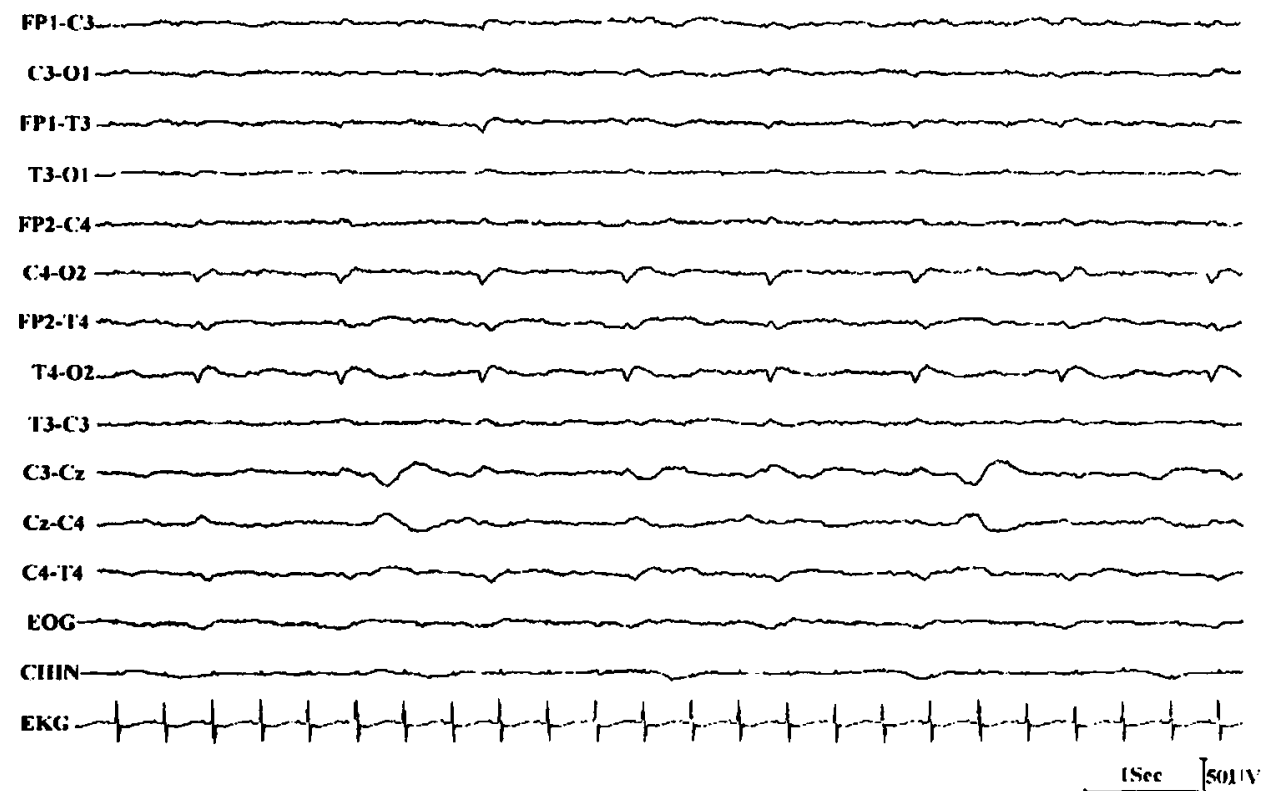

Figure 34 EEG of a 5-day-old neonate on ventilator, showing "depressed brain seizure" characterized by less than one per second, low-amplitude sharp waves over the right hemisphere.

tern ${ }^{111}$ and is usually associated with subtle seizures, tonic or myoclonic seizures, or no behavioral clinical change. This ictal pattern is associated with severe CNS dysfunction and correlates with a poor outcome.

4. Multifocal ictal pattern is characterized by an abnormal background activity and the development of an ictal pattern independently or, rarely, simultaneously over two or more areas of one or both hemispheres. Two or more focal seizures may appear concomitantly in the same or, more commonly, the opposite hemisphere and appear to progress independently. This ictal EEG pattern is usually associated with subtle seizures; the underlying pathology consists of severe encephalopathy due to infection, congenital anom-

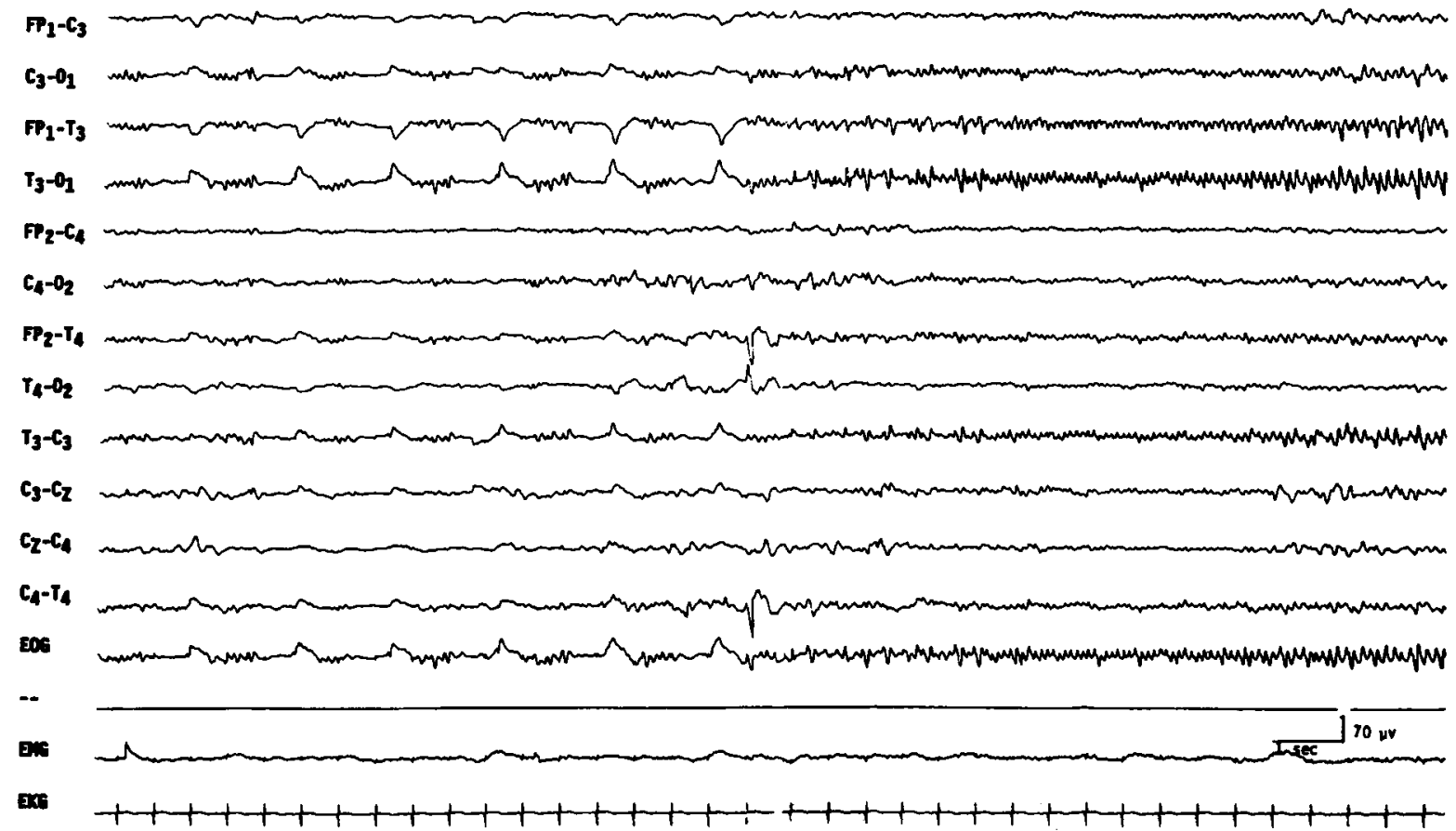

Figure 35 EEG of a 3-day-old comatose neonate with history of seizures, showing an electrographic "alpha band" seizure pattern without clinical accompaniment. 
alies, birth trauma, or anoxia. This pattern carries a poor prognosis for normal neurologic development.

\section{Technical aspects}

Several technical points are of crucial importance to optimize neonatal EEG recording. The study should be long enough to include active and quiet sleep; the total duration of the recording may exceed the usual 30 minutes recommended in adults. In most neonates it may be necessary to record the EEG for 45 to 60 minutes. The presence of sleep differentiation is an important maturational feature for EEG interpretation. Some abnormal patterns such as the degree of discontinuity, asynchrony and asymmetry, presence of multifocal sharp transients, or delta brushes can be evaluated only in quiet sleep. Additionally, polygraphic variables must be routinely recorded in addition to several channels of scalp EEG. These include respiration, extraocular movements, EKG, and chin activity. These non-EEG variables are critical in identifying different states (awake, active sleep, or quiet sleep) and recognition of various artifacts. A neonatal EEG lacking such polygraphic variables is difficult to interpret unless it is grossly abnormal.

\section{EEG IN STATUS EPILEPTICUS}

Status epilepticus (SE) is usually defined as continuous seizure activity persisting for more than 30 minutes or more than one sequential seizure without full recovery of consciousness between seizures. A very common reason for ordering an emergency EEG is for the diagnosis and management of SE. A simplified classification of SE includes: (1) generalized convulsive status, characterized by motor seizures with loss of consciousness; (2) simple partial or focal status, characterized by focal motor seizures repeating frequently or epilepsia partialis continua with the patient remaining fully conscious; and (3) nonconvulsive status (NCSE) characterized by a variable alteration of consciousness with minimal or no motor activity.

NCSE poses many challenging nosologic, diagnostic, and therapeutic problems. NCSE includes: (1) absence status, occurring in the setting of generalized epilepsy (idiopathic or symptomatic) and (2) complex partial status associated with focal or partial epilepsy of frontal or temporal onset. In both types, the patient may present with mental status alteration (e.g., slowness in behavior and mentation, confusion, and, rarely, stupor or coma). Then, there are patients who after treatment of generalized convulsive status continue to be obtunded or comatose and show epileptiform discharges in their EEG. These patients are often designated as having "subtle" SE or lumped under NCSE.
It is relatively easy to diagnose NCSE associated with focal epilepsy when there are frequent electrographic focal seizures with an ictal EEG pattern that evolves over time with change in the amplitude, frequency, and spatial distribution. However, it is quite common for the ictal EEG pattern associated with complex partial status associated with focal epilepsy to be generalized spikes or sharp waves repeating at 1 to $6 \mathrm{~Hz}$ frequency. Such a generalized EEG pattern is similar to that seen in typical absence status associated with idiopathic generalized epilepsy (absence epilepsy) and atypical absence status in children with secondary generalized epilepsy of the Lennox-Gastaut type. To complicate the situation even further, patients with Lennox-Gastaut syndrome interictally have generalized 1.0 to $3.0 \mathrm{cps}$ spike wave discharges that may be very frequent, and one needs to decide if they represent an ictal pattern (hence atypical absence status) or simply represent a prominent interictal pattern. Some waxing or waning of such generalized epileptiform discharges may not help in the distinction because this may be simply related to state changes.

Some helpful criteria are proposed by Young et al ${ }^{122}$ in patients who show almost continuously occurring generalized, nonevolving epileptiform discharges in their EEGs, including repetitive generalized or focal epileptiform discharges (spikes, sharp waves, and spike waves) that repeat at a rate faster than three per second, very likely represent an ictal pattern. Such repetitive discharges at a frequency slower than three per second are likely to be ictal if significant clinical and/or EEG improvement is demonstrated following small doses of intravenous lorazepam or diazepam. Rhythmic sinusoidal waves of any frequency (ranging from $\beta$ to $\delta$ frequency) may represent an ictal pattern if there is an evolving pattern at the onset (increasing amplitude and/or decreasing frequency) or a decrement pattern at the termination (decremental amplitude or frequency) or postdischarge slowing or voltage attenuation.

In a patient with obtundation or mental status change of recent onset, an EEG is indicated to rule out NCSE. If repetitive generalized epileptiform discharges are recorded in the EEG, 1 to $2 \mathrm{mg}$ of lorazepam or 5 to $10 \mathrm{mg}$ of diazepam are injected intravenously while the EEG is running. A marked clinical improvement of obtundation and disappearance of generalized paroxysmal activity in the EEG would strongly support the diagnosis of NCSE (Figs. 36 and 37). Such a rewarding experience is most common in typical absence status and less common in other forms of NCSE.

Reviewing the previous EEG and obtaining follow-up EEG studies also provide a helpful distinction between ictal and interictal basis for the repetitive generalized spike wave discharges seen in children with 


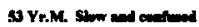

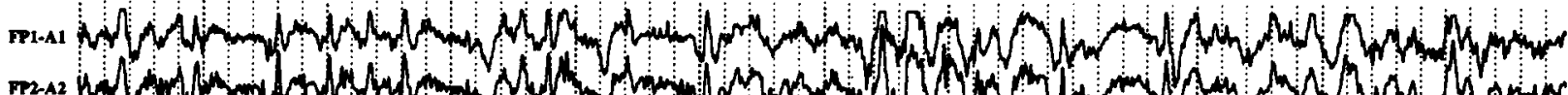

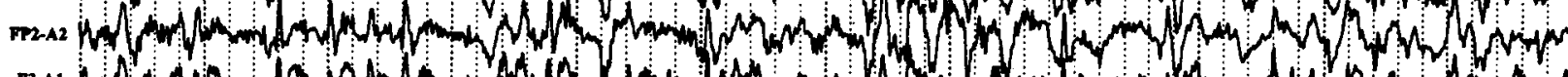

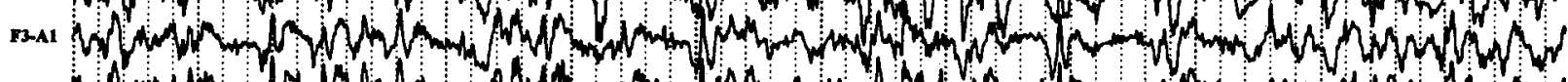

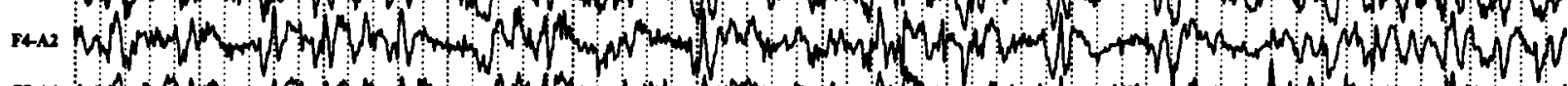

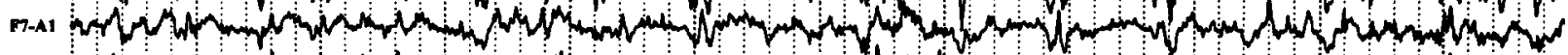

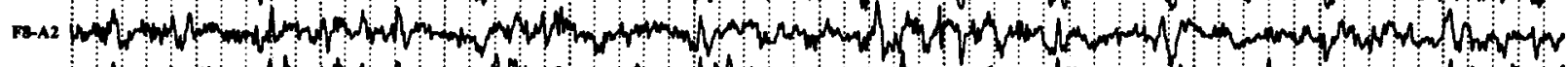

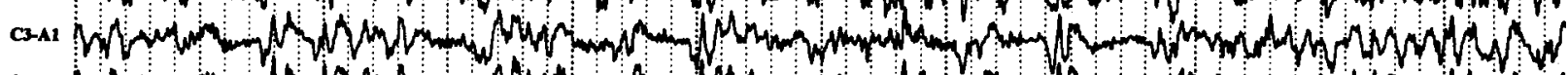

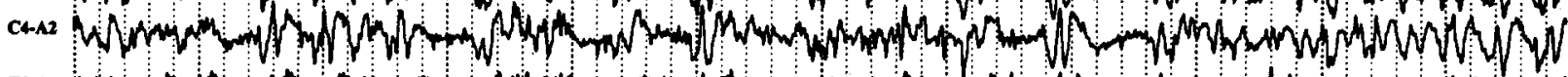

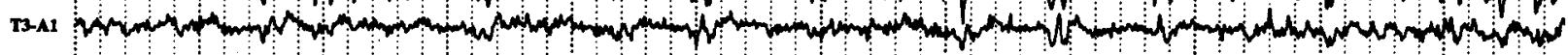

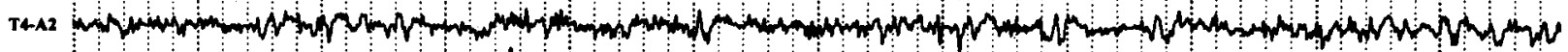

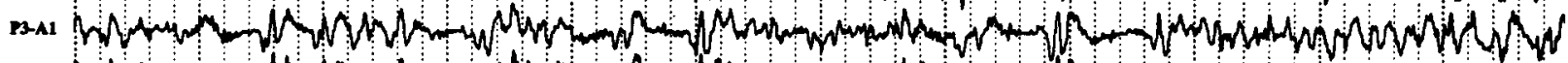

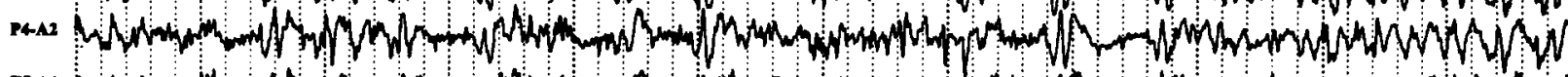

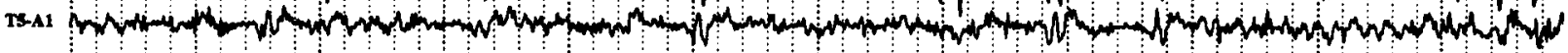

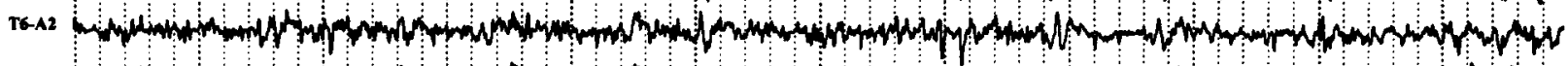

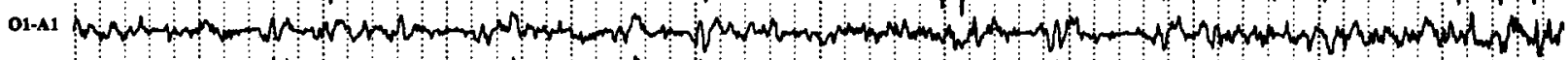

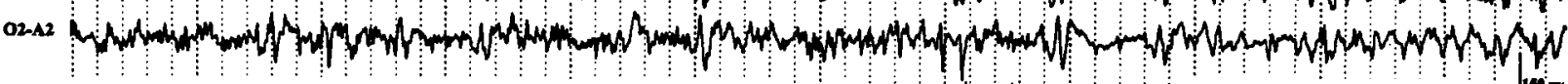

Figure 36 EEG of a 53-year-old man with one day history of acute confusion and slowness of motor responses, showing almost continuous generalized spike wave activity.

TP1-A1 S3Y.M.M. FP2-A2 1thom

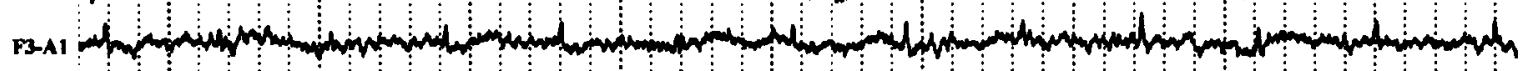

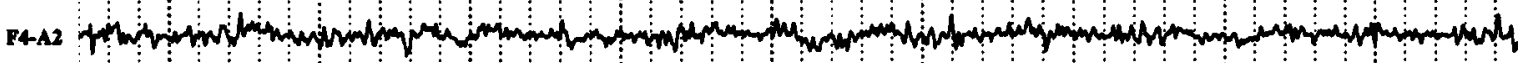
77-A1 Mon

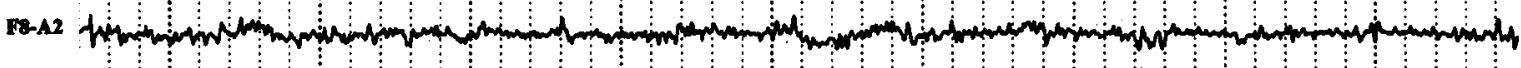

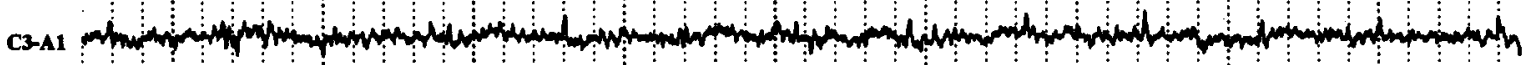

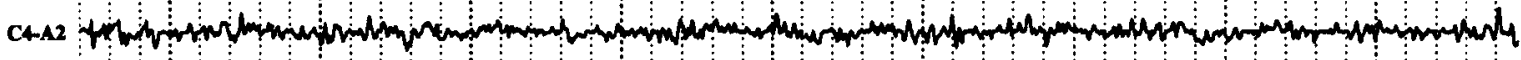
T3-A1 Ath

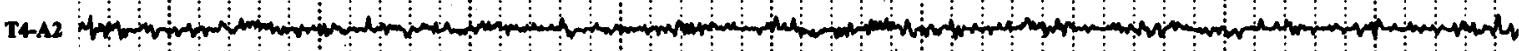

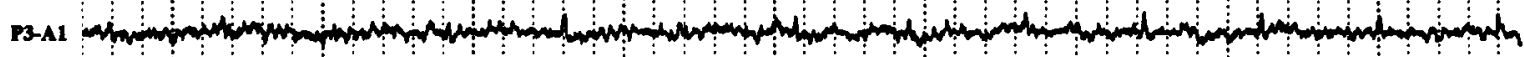

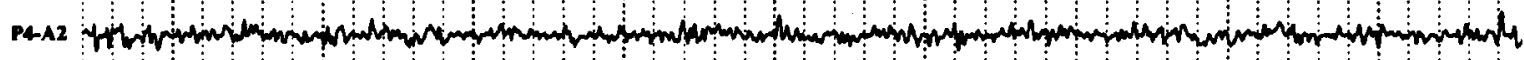
TS-A1

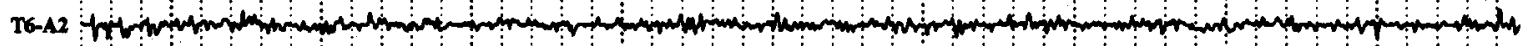
01-A1

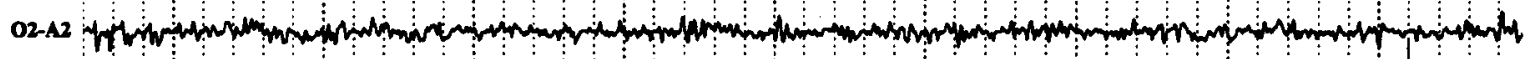
Afwer $4 \mathrm{mg} I V$

185c

Figure 37 EEG of the same patient seen in Figure 36 following $4 \mathrm{mg}$ of intravenous lorazepam, showing disappearance of all paroxysmal activity and mental clearing, highly suggestive of nonconvulsive status epilepticus. 
Lennox-Gastaut syndrome. A period of frequent repetitive generalized spike wave discharges associated with clinical deterioration of mental status is more likely an episode of atypical absence status, particularly if the previous EEGs or follow-up EEGs display dramatically fewer epileptiform abnormalities.

"Subtle" SE commonly includes patients who had a known episode of convulsive or generalized tonicclonic status, brought under control by intravenous antiepileptic therapy (e.g., phenytoin, lorazepam, and barbiturates), but continue to remain obtunded or comatose without significant motor activity. EEG of such patients often show repetitive discharges, which may include lateralized periodic discharges (e.g., PLEDs or BiIPLEDS) or generalized periodic discharges (PEDs). Some epileptologists ${ }^{123}$ are of the opinion that progressive sequential EEG changes occur during generalized convulsive SE with an "intermediary" pattern of PEDs and PLEDs (unilateral or bilateral) before disappearance of all paroxysmal EEG activities, and that the presence of these "intermediary" EEG patterns necessitate further aggressive therapeutic measures (e.g., inducing pentobarbital coma, etc.). Such views are not universal. Many, on the other hand, consider PED and PLED patterns observed during the course of convulsive status not an ictal pattern but suggestive of a severe epileptic encephalopathy reflective of a neuronal dysfunction from underlying brain damage. ${ }^{124}$

Refractory SE is usually treated by continuous intravenous anesthesia maintained by pentobarbital, propofol, or medazolam. The dose is regulated such as to control all clear-cut clinical or electrographic seizures and to maintain a suppression-burst pattern in the EEG. Therefore, continuous bedside EEG is monitored. There is no consensus as to the duration of "burst" and "flat" periods for optimal dosing. Most consider that establishing and maintaining any degree of suppression-burst pattern is adequate.

One final note of caution is that focal motor seizures including epilepsia partialis continua may not show ictal changes in the EEG because of the limited size of neuronal tissue involved during the epileptic seizure or because the ictal pattern in the EEG may be obscured by artifacts. Careful review of the EEG using different montages (especially a transverse bipolar montage going through the midline electrodes) and use of appropriate muscle filters may reveal a low-amplitude ictal pattern.

\section{REFERENCES}

1. Markand ON. Alpha rhythms. J Clin Neurophysiol 1990; 7:163-189

2. Adams A. Studies on the flat electroencephalogram in man. Electroencephalogr Clin Neurophysiol 1959;11:35-41

3. Obrist WD. The electroencephalogram of normal aged adults. Electroencephalogr Clin Neurophysiol 1954;6:235244
4. Busse EM, Obrist WD. Significance of focal electroencephalographic changes in the elderly. Postgrad Med 1963;34: 179-182

5. Orbrist WD. Problems of aging. In: Remond A, ed. Handbook of Electroencephalography and Clinical Neurophysiology. Vol. 6A. Amsterdam: Elsevier; 1976:275-292

6. Hubbard O, Sunde D, Goldenshon ES. The EEG changes in centenarians. Electroencephalogr Clin Neurophysiol 1976;40:407-417

7. Torres F, Faovo A, Loewenson R, et al. The electroencephalogram of elderly subjects revisited. Electroencephalogr Clin Neurophysiol 1983;56:391-398

8. Kooi KA, Gurener AM, Tupper CJ, et al. Electroencephalographic patterns of the temporal region in normal adults. Neurology 1964;14:1029-1035

9. Katz RI, Horowitz GR. Electroencephalogram in septuagenarian: studies in a normal geriatric population. J Am Geriatr Soc 1982;3:273-275

10. Markand ON. Electroencephalography in diffuse encephalopathies. J Clin Neurophysiol 1984;1:357-407

11. Hockaday JM, Potts F, Epstein E, et al. Electroencephalographic changes in acute cerebral anoxia from cardiac or respiratory arrest. Electroencephalogr Clin Neurophysiol 1965; 18:575-586

12. Prior PF. The EEG in Acute Cerebral Anoxia. Amsterdam: Excerpta Medica; 1973

13. Cordeau JP. Monorhythmic frontal delta activity in human electroencephalogram: a study of 100 cases. Electroencephalogr Clin Neurophysiol 1959;11:733-746

14. Schaul N, Lüders H, Sachdev K. Generalized, bilaterally synchronous bursts of slow waves in the EEG. Arch Neurol 1981;38:690-692

15. Bickford RG, Butt HR. Hepatic coma: the electroencephalographic pattern. J Clin Invest 1955;34:790-799

16. Simsarian JP, Harner RN. Diagnosis of metabolic encephalopathy: significance of triphasic waves in the electroencephalogram [abstract]. Neurology 1972;22:456

17. Karnze DS, Bickford RG. Triphasic waves: a reassessment of their significance. Electroencephalogr Clin Neurophysiol 1984;57:193-198

18. Fisch BJ, Klass DW. The diagnostic specificity of triphasic wave patterns. Electroencephalogr Clin Neurophysiol 1988; $70: 1-8$

19. Reiher J. The electroencephalogram in the investigation of metabolic comas. Electroencephalogr Clin Neurophysiol 1970;28:104

20. Bahmon-Dussan JE, Celesia GG, Grigg-Damberger MM. Prognostic significance of EEG triphasic waves in patients with altered states of consciousness. J Clin Neurophysiol 1989;6:313-319

21. MacGillivray BB, Kennedy J. The "triphasic waves" of hepatic encephalopathy [abstract]. Electroencephalogr Clin Neurophysiol 1970;28:428P

22. Aoki Y, Lombroso CT. Prognostic value of electroencephalography in Reye's syndrome. Neurology 1973;23: 333-343

23. Yamada T, Young S, Kimura J. Significance of positive spike bursts in Reye's syndrome. Arch Neurol 1977;34:376-380

24. Drury I. 14-and-6 positive bursts in childhood encephalopathies. Electroencephalogr Clin Neurophysiol 1989;72: 479-485

25. Carroll WM, Mastaglia FL. Alpha and beta coma in drug intoxication uncomplicated by cerebral hypoxia. Electroencephalogr Clin Neurophysiol 1979;46:95-105

26. Kuroiwa Y, Furukawa T, Inaki K. Recovery from drug-induced alpha-coma. Neurology 1981;31:1359-1361 
27. Stockard TJ, Werner SS, Albers JA, et al. EEG findings in phencyclidine intoxication. Arch Neurol 1976;33:200-203

28. Kuroiwa Y, Celesia GG, Chung HD. Periodic EEG discharges and status spongiosus of the cerebral cortex in anoxic encephalopathy: a necropsy case report. J Neurol Neurosurg Psychiatry 1982;45:740-742

29. Paz D, Brenner RD. Bilateral independent periodic lateralized epileptiform discharges, clinical significance. Arch Neurol 1981;38:713-715

30. McCarty GE, Marshall DW. Transient eyelid opening associated with postanoxic EEG suppression-burst pattern. Arch Neurol 1981;38:754-756

31. Reeves AL, Westmoreland BF, Klass DW. Clinical accompaniments of the burst-suppression EEG pattern. J Clin Neurophysiol 1997;14:150-153

32. Chokroverty S. "Alpha-like" rhythms in electroencephalograms in coma after cardiac arrest. Neurology 1975;25:655663

33. Westmoreland BF, Klass DW, Sharbrough FW, et al. "Alpha coma:" electroencephalographic, clinical, pathological and etiological correlation. Arch Neurol 1975;32:713-718

34. Alving J, Miller M, Sindrup E, et al. Alpha-pattern coma following cerebral anoxia. Electroencephalogr Clin Neurophysiol 1979;47:95-101

35. Kuroiwa Y, Furukawa T. EEG prognostication in drug related alpha coma [letter]. Arch Neurol 1981;38:200

36. Chatrian GE, White LE Jr, Shaw CM. EEG pattern resembling wakefulness in unresponsive decerebrate state following traumatic brainstem infarct. Electroenceph Clin Neurophysiol 1964;16:285-289

37. American EEG Society. Guideline three: minimum technical standards for EEG recording in suspected cerebral death. J Clin Neurophysiol 1994;11:10-13

38. Report of Special Task Force. Guidelines for the determination of brain death in children. Pediatrics 1987;80:298-300

39. Markand ON. EEG in the diagnosis of CNS infections. In: Roos KL, ed. Central Nervous System Infectious Diseases and Therapy. New York: Marcel Dekker; 1997:667-689

40. Illis LS, Taylor RM. The electroencephalogram in herpes simplex encephalitis. Lancet 1972;1:718-721

41. Gupta PC, Seth P. Periodic complex in herpes simplex encephalitis: a clinical and experimental study. Electroencephalogr Clin Neurophysiol 1973;35:67-74

42. Chien LT, Boehm RM, Robinson H, et al. Characteristic early electroencephalographic changes in herpes simplex encephalitis: clinical and virologic studies. Arch Neurol 1977; 34:361-364

43. Upton A, Gumpert J. Electroencephalography in the diagnosis of herpes simplex encephalitis. Lancet 1970;1:650-652

44. Markand ON, Daly DD. Pseudoperiodic lateralized paroxysmal discharges in electroencephalogram. Neurology 1971; 21:975-981

45. Cobb W, Hill D. Electroencephalogram in subacute progressive encephalitis. Brain 1950;73:392-404

46. Cobb W. The periodic events of subacute sclerosing leukoencephalitis. Electroenceph Clin Neurophysiol 1966; 21:278-294

47. Markand ON, Panszi JG. The electroencephalogram in subacute sclerosing panencephalitis. Arch Neurol 1975;32:719726

48. Westmoreland BF, Gomex MR, Blume WT. Activation of periodic complexes of subacute sclerosing panencephalitis by sleep. Ann Neurol 1977;1:185-187

49. Burger LJ, Rowan J, Goldenshon E. Creutzfeldt-Jakob disease. Arch Neurol 1972;26:428-433
50. Au WJ, Gabor AJ, Viyan N, Markand ON. Periodic lateralized epileptiform complexes (PLEDs) in Creutzfeldt-Jakob disease. Neurology 1980;30:611-617

51. Furlan A, Henry CE, Sweeny PI, et al. Focal EEG abnormalities in Heidenhain's variant of Jakob-Creutzfeldt disease. Arch Neurol 1981;38:312-314

52. Chiofalo N, Fuentes A, Galvez S. Serial EEG findings in 27 cases of Creutzfeldt-Jakob disease. Arch Neurol 1980;37: 143-145

53. Chiappa KH, Burke CJ, Young RR. Creutzfeldt-Jakob disease: periodic sharp waves in the EEG as an invariant element of the clinical syndrome. Presented at the American Academy of Neurology Meeting, Chicago, 1979

54. Gloor P, Kalaby O, Giard N. The electroencephalogram in diffuse encephalopathies: electroencephalographic correlates of gray and white matter lesion. Brain 1968;91:779-802

55. Pampiglione G, Harden A. Neurophysiological identification of a late infantile form of "neuronal lipidosis." J Neurol Neurosurg Psychiatry 1973;36:68-74

56. Letemendia F, Pampiglione G. Clinical and EEG observation in Alzheimer's disease. J Neurol Neurosurg Psychiatry 1958;21:167-172

57. Liddell DW. Investigation of EEG findings in presenile dementia. J Neurol Neurosurg Psychiatry 1958;21:173-176

58. Gordon EB, Sim M. The EEG in presenile dementia. J Neurol Neurosurg Psychiatry 1967;30:285-291

59. Muller HF, Kral VA. The electroencephalogram in advanced senile dementia. J Am Geriatr Soc 1967;15:415-426

60. Margerison JH, Scott DF. Huntington's chorea: clinical EEG and neuropathological findings [abstract]. Electroencephalogr Clin Neurophysiol 1965;19:314

61. Daly DD, Markand ON: Focal brain lesions. In: Daly DD, Pedley TA, eds. Current Practice of Clinical Electroencephalography. 2nd ed. New York: Raven Press; 1990:335370

62. Walter WG. The location of cerebral tumours by electroencephalography. Lancet 1936;11:305-308

63. Arfel G, Fischgold H. EEG-signs in tumours of the brain. Electroencephalogr Clin Neurophysiol 1961;19(suppl):3650

64. Brenner R, Sharbrough F. Electroencephalographic evaluation in Sturge-Weber syndrome. Neurology 1976;26:629-632

65. Kershmann J, Conde A, Gibson WC. Electroencephalography in differential diagnosis of supratentorial tumors. Arch Neurol Psychiatry (Chicago) 1949;62:255-268

66. Cobb WA, Guillof RJ, Cast J. Breach rhythm: the EEG related to skull defects. Electroencephalogr Clin Neurophysiol 1979;47:251-271

67. Chatrian GE, Shaw CM, Leftman H. The significance of periodic lateralized epileptiform discharges in EEG: an electrographic, clinical and pathological study. Electroencephalogr Clin Neurophysiol 1964;17:177-193

68. Maulsby RL. Some guidelines for assessment of spikes and sharp waves in EEG tracings. Am J EEG Technol 1971; $11: 3-16$

69. Gloor P. The EEG and differential diagnosis of epilepsy. In: Van Duijn H, Donker DNJ, Van Huffelen AC, eds. Current Concepts in Clinical Neurophysiology. The Netherlands: NV Drukkerij Trio; 1977:9-21

70. Petersen I, Eeg-Olofsson O, Sellden U. Paroxysmal activity in EEG of normal children. In: Kellaway P, Petersen I, eds. Clinical Electroencephalography of Children. New York: Grune \& Stratton; 1968:167-188

71. Eeg-Olofsson O, Petersen I, Sellden U. The development of the electroencephalogram in normal children from the age 
of one through fifteen years: paroxysmal activity. Neuropediatrie 1971;4:375-404

72. Zivin L, Ajmone-Marsan C. Incidence and prognostic significance of "epileptiform" activity in the EEG of nonepileptic subjects. Brain 1968;91:751-778

73. Goodin DS, Aminoff MJ. Does the interictal EEG have a role in the diagnosis of epilepsy. Lancet 1984;1:837-839

74. Bridgers SL. Epileptiform abnormalities discovered on electroencephalographic screening of psychiatric inpatients. Arch Neurol 1987;44:312-316

75. Gregory RP, Oates T, Merry RTG. Electroencephalogram epileptiform abnormalities in candidates for aircrew training. Electroencephalogr Clin Neurophysiol 1993;86:75-77

76. Salinsky M, Kanter R, Dasheiff R. Effectiveness of multiple EEGs in supporting the diagnosis of epilepsy: an operational curve. Epilepsia 1987;28:331-334

77. Gotman J, Marciani MG. Electroencephalographic spiking activity, drug levels and seizure occurrence in epileptic patients. Ann Neurol 1985;17:597-603

78. Reilly EL, Peters JF. Relationship of some varieties of electroencephalographic photosensitivity to clinical convulsive disorders. Neurology 1973;23:1050-1057

79. Puglia JF, Brenner RP, Soso MJ. Relationship between prolonged and self-limited photoparoxysmal responses and seizure incidence: study and review. J Clin Neurophysiol 1992;9:137-144

80. Wolf P, Gooses R. Relation of photosensitivity to epileptic syndromes. J Neurol Neurosurg Psychiatry 1986;49:13861391

81. Silverman D. The anterior temporal electrode and the tentwenty system. Electroencephalogr Clin Neurophysiol 1960; 12:735-737

82. Goodin DA, Aminoff MJ, Laxer KD. Detection of epileptiform activity by different noninvasive EEG methods in complex partial epilepsy. Ann Neurol 1990;27:330-334

83. Markand ON. Psychogenic seizures (pseudoseizures). In: Radhakrishnan K, ed. Medically Refractory Epilepsy. Trivandrum, India: Sree Chitra Tirunal Institute of Medical Sciences and Technology; 1999:61-75

84. Gabor AJ, Ajmone-Marsan C. Co-existence of focal and bilateral diffuse paroxysmal discharges in epileptics. Epilepsia 1969;10:453-472

85. Lombroso CT. Consistent EEG focalities detected in subjects with primary generalized epilepsies monitored for two decades. Epilepsia 1997;38:797-812

86. Jeavons PM, Bower BD. Infantile spasms. In: Vinken PJ, Bruyn GW, eds. Handbook of Clinical Neurology. Vol. 15, The Epilepsies. New York: American Elsevier; 1974:219-234

87. Markand ON, Garg BP, Brandt IK. Nonketotic hyperglycenemia: electroencephalographic and evoked potential abnormalities. Neurology 1982;32:151-156

88. Fariello RG, Chun RW, Doro JM, Buncic JR, Pritchard JS. EEG recognition of Aicardi's syndrome. Arch Neurol 1977; 34:563-566

89. Blume WT, David RB, Gomez MR. Generalized sharp and slow wave complexes: associated clinical features and longterm follow-up. Brain 1973;96:289-306

90. Markand ON. Slow spike wave activity in EEG and associated clinical features: often called "Lennox" or "LennoxGastaut" syndrome. Neurology 1977;27:746-757

91. Noriega-Sanchez A, Markand ON. Clinical and electroencephalographic correlation of independent multifocal spike discharges. Neurology 1976;26:667-672
92. Blume WT. Clinical and electrographic correlates of the multiple independent spike foci pattern in children. Ann Neurol 1978;4:541-547

93. Brenner RP, Atkinson R. Generalized paroxysmal fast activity: electroencephalographic and clinical features. Ann Neurol 1981;11:386-390

94. Gabor AJ, Seyal M. Effect of sleep on the electrographic manifestations of epilepsy. J Clin Neurophysiol 1986;3:23-38

95. Williamson PD, Spencer DD, Spencer SS, Novelly RA, Mattson RH. Complex partial seizures of frontal lobe origin. Ann Neurol 1985;18:497-504

96. Morris HH, Dinner DD, Lüders H. Supplementary motor seizures: clinical and electrographic findings. Neurology 1988;38:1075-1082

97. Blom S, Heijbel J. Benign epilepsy of children with centrotemporal EEG foci: discharge rates during sleep. Epilepsia 1975;16:133-140

98. Gregory DL, Wong PK. Topographical analysis of the centro-temporal discharges in benign Rolandic epilepsy of childhood. Epilepsia 1984;25:705-711

99. Lerman P, Kivity S. Benign focal epilepsy of childhood: a follow-up study of 100 recovered patients. Arch Neurol 1975; 32:261-264

100. Pedley TA, Tharp BR, Herman K. Clinical and electroencephalographic characteristics of midline parasagittal foci. Ann Neurol 1981;9:142-149

101. Pourmand RA, Markand ON, Thomas C. Midline spike discharges: clinical and EEG correlations. Clin Electroencephalogr 1984;15:232-237

102. Fischer RA, Clancy RR. Midline foci of epileptiform activity in children and neonates. J Child Neurol 1987;2: 224-228

103. Klass DW, Westmoreland BF. Nonepileptogenic epileptiform electroencephalographic activity. Ann Neurol 1985;18: 627-635

104. White JC, Langston JW, Pedley TA. Benign epileptiform transients of sleep: clarification of the "small sharp spike" controversy. Neurology 1977;27:1061-1068

105. Thomas JE, Klass DW. Six-per-second spike and wave pattern in the electroencephalogram. Neurology 1968;18:587593

106. Hughes JR. Two forms of the $6 / \mathrm{sec}$ spike and wave complex. Electroencephalogr Clin Neurophysiol 1980;48:535550

107. Lipman IL, Hughes JR. Rhythmic midtemporal discharges: an electroclinical study. Electroencephalogr Clin Neurophysiol 1969;27:43-47

108. Reiher J, Lebel M. Wicket spikes: clinical correlates of a previously undescribed EEG pattern. Can J Neurol Sci 1977;4:39-47

109. Westmoreland BF, Klass DW. A distinctive rhythmic EEG discharge of adults. Electroencephalogr Clin Neurophysiol 1981;51:186-191

110. Westmoreland BF, Klass DW. Unusual variants of subclinical rhythmic electrographic discharge of adults (SREDA). Electroencephalogr Clin Neurophysiol 1997; 102:1-4

111. Lombroso CT. Neonatal polygraphy in full-term and premature infants: a review of normal and abnormal findings. J Clin Neurophysiol 1985;2:105-155

112. Aso K, Scher MS, Barmada MA. Neonatal electroencephalography and neuropathology. J Clin Neurophysiol 1989;6:103-123 
113. Ellingson RJ, Peters JP. Development of EEG and daytime sleep patterns in normal full-term infants during the first three months of life: longitudinal observations. Electroencephalogr Clin Neurophysiol 1980;49:112-124

114. Cukier F, Andre M, Monod N, Dreyfus-Brisac C. Apport de I'EEG au diagnostic des hemorragies intra-ventriculaires du premature. Rev Electroencephalogr Neurophysiol 1972; 2:318-322

115. Clancy R, Tharp B. Positive Rolandic sharp waves in the electroencephalograms of premature neonates with intraventricular hemorrhage. Electroencephalogr Clin Neurophysiol 1984;57:395-404

116. Marret S, Parain D, Samson-Dollfus D. Positive Rolandic sharp waves and periventricular leukomalacia in the newborn. Neuropediatrics 1986;17:199-202

117. Novotny EJ, Tharp BR, Coen RW, Bejar R, Enzmann D, Vaucher YE. Positive Rolandic sharp waves in the EEG of the premature infant. Neurology 1987;37:1481-1486

118. Monod N, Pajot N, Guidasci S. The neonatal EEG: statistical studies and prognostic value in full-term and pre-term babies. Electroencephalogr Clin Neurophysiol 1972;32: 529-544
119. Lombroso C. Quantified electrographic scales on 10 preterm healthy newborns followed up to 40-43 weeks of conceptional age by serial polygraphic recordings. Electroencephalogr Clin Neurophysiol 1979;46:460-474

120. Tharp BR, Cukier P, Monod N. The prognostic value of the electroencephalogram in premature infants. Electroencephalogr Clin Neurophysiol 1981;51:219-236

121. Hrachovy RA, Mizrahi EM, Kellaway P. Electroencephalography of newborn. In: Daly DD, Pedley TA, eds. Current Practice of Clinical Electrophysiology. New York: Raven Press; 1990:201-242

122. Young GB, Jordan KG, Dowig GS. An assessment of nonconvulsive seizures in the intensive care unit using continuous EEG monitoring: an investigation of variables associated with mortality. Neurology 1996;47:83-89

123. Treiman DM, Walton NY, Kendrick C. A progressive sequence of electroencephalographic changes during generalized convulsive status epilepticus. Epilepsy Res 1990;5: 49-60

124. Brenner RP. Is it status? Epilepsia 2002;43(suppl 3):103-113 